\title{
Functional Limit Theorems
}

\author{
by \\ Bin Chen. B.Sc., M.Sc.

\begin{abstract}
A thesis submitted to
the Faculty of Graduate Studies and Research

in partial fulfilment of the requirements for the degree of
\end{abstract}

Doctor of Philosophy

School of Mathematics and Statistics

Carleton University

Ottawa, Ontario, Canada

December, 1998

(C) Copyright 1998

Bin Chen 
National Library of Canada

Acquisitions and Bibliographic Services

395 Wellington Street Ottawa ON KIA ON4 Canada
Bibliothèque nationale du Canada

Acquisitions et services bibliographiques

395, rue Wellington

Ottawa ON KIA ON4

Canada
The author has granted a nonexclusive licence allowing the National Library of Canada to reproduce, loan, distribute or sell copies of this thesis in microform, paper or electronic formats.
L'auteur a accordé une licence non exclusive permettant à la Bibliothèque nationale du Canada de reproduire, prêter, distribuer ou vendre des copies de cette thèse sous la forme de microfiche/film, de reproduction sur papier ou sur format électronique.

The author retains ownership of the copyright in this thesis. Neither the thesis nor substantial extracts from it may be printed or otherwise reproduced without the author's permission.
L'auteur conserve la propriété du droit d'auteur qui protège cette thèse. $\mathrm{Ni}$ la thèse ni des extraits substantiels de celle-ci ne doivent être imprimés ou autrement reproduits sans son autorisation. 


\section{Abstract}

Since Strassen (1964) proved his functional law of the iterated logarithm, functional limit theorems have been very active areas of research in probability and statistics. This dissertation is devoted to this topic.

In Chapter 1 we give an introduction to our main topics.

In Chapter 2, we first establish a large deviation for increments of a Wiener process. A functional modulus of continuity for a Wiener process is presented and proved using this large deviation.

In Chapter 3, functional limit theorems for Csörgö-Révész increments both for a Wiener process and partial sums of i.i.d.r.v. are discussed, and some new results are presented and proved. In paticular, we refine a functional limit theorem for the Erdös and Rényi law of large numbers which, unlike previous ones, really implies the classic Erdös and Rényi law of large numbers.

In Chapter 4, we present, discuss and prove functional limit theorems for subsequences of increments for a Wiener process. Our study constitutes an extension of results of Gut (1986) and Vasudeva and Savitha (1993).

In Chapter 5, we move our attention to a two-parameter Wiener process. A detailed discussion of Strassen-type laws of the iterated logarithm for two-parameter Gaussian processes are given. Our results contain most of the Strassen type laws for one-parameter and two-parameter Wiener processes.

Motivated by change-point analysis, Chapter 6 is devoted to large deviations for two-parameter Wiener, Kiefer and "tied down" processes in weighted metric.

Finally, in Chapter 7, we study Brownian local time processes, including its increments, large deviations, functional limit theorems and small time Strassen laws. 


\section{Acknowledgements}

I would like to express my sincere and deep gratitude to my thesis supervisor, Professor Miklós Csörgö for his invaluable supervision and encouragement during the research of this thesis. as well as for his kindness and support during the past five years. I would also like to thank Professor Barbara Szyszkowicz for her support and encouragement during my study.

I am very grateful to Carleton University and to the Ontario Graduate Scholarship program for providing me with financial support during my $\mathrm{Ph}$.D. studies and to the School of Mathematics and Statistics for the strong academic atmosphere from which I have greatly benefited.

Last, but not the least, I wish to thank my parents, my wife Xiaojian and my son Weibo for their love, understanding, support and constant encouragement. 


\section{Contents}

1 Introduction 1

2 A Functional Modulus of Continuity for Wiener Processes 10

2.1 A Modulus of Continuity for a Wiener Process . . . . . . . . . 10

2.2 Large Deviations . . . . . . . . . . . . . . . . . . 11

2.3 A Functional Modulus of Continuity . . . . . . . . . . 14

2.4 A Note . . . . . . . . . . . . . . . . . . . . . . . 19

3 Csörgö-Révész Increments $\quad 21$

3.1 Csörgö-Révész Increments of a Wiener Process . . . . . . . . . . . . 21

3.2 Functional Limit Theorems for Csörgö-Révész Increments of a Wiener Process . . . . . . . . . . . . . . . . . . . 24

3.3 Functional Limit Theorems for Partial Sums of I.I.D.R.V. . . . . . 34

3.4 A Functional Limit Theorem for the Erdős and Rényi Law of Large Numbers . . . . . . . . . . . . . . . . . . . . . 37

4 Subsequences of Increments for a Wiener Process 41

4.1 The Laws of the Iterated Logarithm for Subsequences . . . . . . . 41

4.2 How Large Are Subsequences of Increments for a Wiener Process . . 42

4.3 Functional Strong Limit Theorems . . . . . . . . . . . . 45 
5 Two-Parameter Wiener Processes $\quad 54$

5.1 Introduction . . . . . . . . . . . . . . . 54

5.2 A Large Deviation for the Two-Parameter Wiener Process ..... . 55

5.3 Strassen-Type Laws of the Iterated Logarithm for the Two-Parameter Wiener Process ...................... 60

5.4 Strassen-Type Laws of the Iterated Logarithm for the Kiefer Process and the Empirical Process .................. 70

6 Large Deviations for Two-Parameter Gaussian Processes Related to $\begin{array}{ll}\text { Change-Point Analysis } & 75\end{array}$

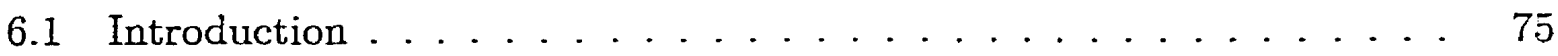

6.2 Large deviations for Brownian sheet in $q$-metric . . . . . . . . 78

6.3 Large deviations for Kiefer processes . . . . . . . . . . . . 85

6.4 Large deviations for "tied down" Kiefer processes . . . . . . . . . 87

7 Brownian Local Time Processes $\quad 91$

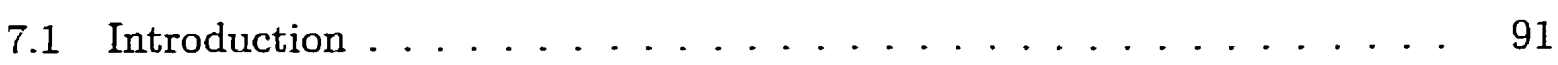

7.2 Increments of the Brownian Local Time Process . . . . . . . . . . . 92

7.3 A Large Deviation . . . . . . . . . . . . . . . . 98

7.4 Functional Limit Theorems . . . . . . . . . . . . . . 104

7.5 The Small Time Strassen Law . . . . . . . . . . . . . . 110

$\begin{array}{lr}\text { Bibliography } & 112\end{array}$ 


\section{Chapter 1}

\section{Introduction}

A stochastic process $\{W(t ; \omega)=W(t) ; \quad 0 \leq t<\infty\}$, where $\omega \in \Omega$, and $\{\Omega, \mathcal{F}, P\}$ is a probability space, is called a standard Wiener process if

(i) $W(t)-W(s) \in \mathcal{N}(0, t-s)$ ( a normal r.v. with mean zero and variance $t-s$ ) for all $0 \leq s<t<\infty$ and $W(0)=0$.

(ii) $W(t)$ is an independent increment process, that is $W\left(t_{2}\right)-W\left(t_{1}\right), W\left(t_{3}\right)-$ $W\left(t_{2}\right), \cdots, W\left(t_{i+1}\right)-W\left(t_{i}\right)$ are independent r.v. for all $0 \leq t_{1}<t_{2}<\cdots<t_{i}<$ $t_{i+1}<\infty \quad(i=1,2,3, \cdots)$,

(iii) the sample path function $W(t, \omega)$ is continuous in $t$ with probability one.

We note that (i) and (ii) imply that the covariance function of a standard Wiener process is

$$
R(s, t)=E W(s) W(t)=s \wedge t
$$

The existence of this process and continuity, as well as nondifferentiability, of almost all sample path functions of this process was established by N. Wiener (1923). Csörgő and Révész (1981, Chapter 1) gave a constructive proof for the existence and 
relevant path properties of the Wiener process that play an important role in this thesis.

The continuity of the sample path functions of a Wiener process is one of its basic features. Lévy $(1937,1948)$ proved the following important moduli of continuity.

$$
\limsup _{h \rightarrow 0} \sup _{0 \leq t \leq 1-h} \sup _{0<s \leq h} \frac{|W(t+s)-W(t)|}{\sqrt{2 h \log \frac{1}{h}}}=1 \quad \text { a.s.. }
$$

and

$$
\limsup _{h \rightarrow 0} \sup _{0 \leq t \leq 1-h} \frac{|W(t+h)-W(t)|}{\sqrt{2 h \log \frac{1}{h}}}=1 \quad \text { a.s. }
$$

Here limsup $\sup _{h \rightarrow 0}$ can be replaced by $\lim _{h \rightarrow 0}$ (cf., e.g., Csörgö and Révész 1981). As to the non-differentiability of sample path functions of a Wiener process, Csörgö and Révész (1979a) established the following modulus of non-differentiability.

$$
\lim _{h \rightarrow 0} \inf _{0 \leq s \leq 1-h} \sup _{0 \leq t \leq h} \sqrt{\frac{8 \log h^{-1}}{\pi^{2} h}}|W(s+t)-W(s)|=1,
$$

which also implies Wiener's result that almost all sample functions of a Wiener process are nowhere differentiable.

Khinchine's (1933) law of the iterated logrithm for a Wiener process is

$$
\limsup _{T \rightarrow \infty} \frac{W(T)}{\sqrt{2 T \log \log T}}=1 \quad \text { a.s. }
$$

We have also

$$
\limsup _{T \rightarrow \infty} \sup _{0 \leq t \leq T} \frac{W(t)}{\sqrt{2 T \log \log T}}=\limsup _{T \rightarrow \infty} \sup _{0 \leq x \leq 1} \frac{W(T x)}{\sqrt{2 T \log \log T}}=1 \quad \text { a.s. }
$$

(cf., e.g., Csörgö and Révész 1981). Furthermore, a brand new topic, the behaviour of $W(T x), 0 \leq x \leq 1, T \rightarrow \infty$, was first introduced by Strassen (1964). 
Let $S$ be the set of absolutely continuous functions (with respect to Lebesgne measure) such that

$$
f(0)=0 \text { and } \int_{0}^{1}\left(f^{\prime}(x)\right)^{2} d x \leq 1 .
$$

The set $S$ is compact (cf.. e.g.. Stout 1974, Lemma 5.3.2). Define

$$
\xi_{T}(x)=\frac{W(T x)}{\sqrt{2 T \log \log T}}
$$

for $x \in[0,1]$. Then $\left\{\xi_{T}(x), T \geq 3\right\}$ is a set of stochastic processes with sample paths almost surely in $C[0,1]$. In this setup, in sup-norm in $C[0,1]$, Strassen's law is as follows.

Theorem 1.0.1 (Strassen 1964) The set $\left\{\xi_{T}(\cdot), T \geq 3\right\}$ is relatively compact in $\mathrm{C}[0,1]$ with probability one. and the set of its limit points is $S$ (as $T \rightarrow \infty$ ).

It is important to spell out what Theorem 1.0.1 says: Given an arbitrary $\varepsilon>0$, let $S^{\varepsilon}$ denote the functions having distance $<\varepsilon$ from $S$. Then $P\left\{\xi_{T}(\cdot) \in S^{\varepsilon}\right.$ eventually $\}$ $=1$. Moreover, $P\left\{\sup _{0 \leq x \leq 1}\left|\xi_{T}(x)-f(x)\right| \leq \varepsilon\right.$ i.o. for each $\left.f \in S\right\}=1$. In other words, we have that

$$
\lim _{T \rightarrow \infty} \inf _{f \in S} \sup _{0 \leq x \leq 1}\left|\xi_{T}(x)-f(x)\right|=0 \quad \text { a.s. }
$$

and for any $f \in S$

$$
\liminf _{T \rightarrow \infty} \sup _{0 \leq x \leq 1}\left|\xi_{T}(x)-f(x)\right|=0 \quad \text { a.s. }
$$

Remark 1.0.1 Since $|f(1)| \leq 1$ for any function $f \in S$, and $f(x)=x \in S$, Theorem 1.0.1 implies (1.0.4) and (1.0.5).

Strassen's law has been generalized extensively. For example, Gantert (1993) showed that with probability one

$$
\left\{\frac{W(h x)}{\sqrt{2 h \log \log \frac{1}{h}}}, 0 \leq x \leq 1,0<h<1\right\} \subset C[0,1]
$$


to increments of a Wiener process, we refer to Chan. Csörgö and Révész (1978) and Révész (1979).

Let $0<a_{T} \leq T$ satisfy the following conditions:

(i) $a_{T}$ is non-decreasing

(ii) $T a_{T}^{-1}$ is non-decreasing;

(iii) $\lim _{T \rightarrow \infty} \frac{\log T a_{r}^{-1}}{\log \log T}=r, \quad(0 \leq r \leq \infty)$.

Introduce the following notations:

$$
\begin{aligned}
& \beta_{T}=\left(2 a_{T}\left(\log T a_{T}^{-1}+\log \log T\right)\right)^{-\frac{1}{2}}, \\
& Y_{t, T}(x)=\beta_{T}\left(W\left(t+x a_{T}\right)-W(t)\right), \quad 0 \leq x \leq 1, \quad 0 \leq t \leq T-a_{T} .
\end{aligned}
$$

Especially, we are now interested in the following result (Book and Shore 1978):

$$
\liminf _{T \rightarrow \infty} \sup _{0 \leq t \leq T-a_{T}} Y_{t, T}(1)=\liminf \sup _{T \rightarrow \infty} \sup _{0 \leq t \leq T-a_{T}}\left|Y_{t, T}(x)\right|=\alpha(r) \quad \text { a.s. }
$$

where

$$
\alpha(r)= \begin{cases}\sqrt{\frac{r}{r+1}}, & 0 \leq r<\infty, \\ 1, & r=\infty .\end{cases}
$$

We note that the above result is not sharp for the case of $r=0$. Replacing $\beta_{T}$ by $\gamma_{T}$, Shao (1986) proved that under conditions (i). (ii) and

(iv) $\lim _{T \rightarrow \infty}\left(T / a_{T}\right) / \log \log T=\infty$,

we have

$$
\liminf _{T \rightarrow \infty} \sup _{0 \leq t \leq T-a_{T}} Z_{t, T}(1)=\liminf _{T \rightarrow \infty} \sup _{0 \leq t \leq T-a_{T}} \sup _{0 \leq x \leq 1}\left|Z_{t, T}(x)\right|=1 \quad \text { a.s. },
$$

where

$$
\begin{aligned}
& Z_{t, T}(x)=\gamma_{T}\left(W\left(t+x a_{T}\right)-W(t)\right), \quad 0 \leq x \leq 1, \quad 0 \leq t \leq T-a_{T}, \\
& \gamma_{T}=\left(2 a_{T}\left(\log T a_{T}^{-1}-\log \log \log T\right)\right)^{-\frac{1}{2}}
\end{aligned}
$$


In Chapter 3, we first present and prove two functional limit theorems which imply (1.0.10) and (1.0.11), respectively. Üsing strong approximation methods, we extend these two results to partial sums of i.i.d.r.v.. Finally paying attention to the Erdös and Rényi law of large numbers (cf. Erdős and Rényi 1970). we obtain a functional limit theorem, and explain why it is a real generalization of the law.

In Chapter 4, we discuss functional limit theorems for subsequences of increments of a Wiener process. Our study is an extension of results of Gut (1986), and Vasudeva and Savitha (1993).

Let $X(x, y)(0 \leq x \leq \infty, 0 \leq y \leq \infty)$ be a two-parameter stochastic process and consider the rectangle $R=\left[x_{1}, x_{2}\right) \times\left[y_{1}, y_{2}\right) \subset R_{+}^{2}\left(0 \leq x_{1}<x_{2}<\infty, 0 \leq y_{1}<y_{2}<\right.$ $\infty)$. Define the " $X$-measure" $X(R)$ of $R$ by

$$
X(R)=X\left(x_{2}, y_{2}\right)-X\left(x_{1}, y_{2}\right)-X\left(x_{2}, y_{1}\right)+X\left(x_{1}, y_{1}\right)
$$

A stochastic process $\{W(x, y), 0 \leq x \leq \infty, 0 \leq y \leq \infty\}$ is called a standard twoparameter Wiener process if

(i) $W(R) \in \mathcal{N}(0, \lambda(R))$ for all $R=\left[x_{1}, x_{2}\right) \times\left[y_{1}, y_{2}\right) \subset R_{+}^{2}$ and where $\lambda(R)=$ $\left(x_{2}-x_{1}\right)\left(y_{2}-y_{1}\right)$,

(ii) for all $0 \leq x, y<\infty$, with probability one, $W(0, y)=W(x, 0)=0$,

(iii) $W(x, y)$ is an independent increment process, that is $W\left(R_{1}\right), W\left(R_{2}\right), \cdots, W\left(R_{n}\right)$ $(n=2,3, \cdots)$ are independent r.v. if $R_{1}, R_{2}, \cdots, R_{n}$ are disjoint rectangles,

(iv) the sample path function $W(x, y, \omega)$ is continuous in $x, y$ with probability one. 
We note that (i)-(iii) imply that the covariance function of a standard twoparameter Wiener process is

$$
E W\left(x_{1}, y_{1}\right) W\left(x_{2}, y_{2}\right)=\left(x_{1} \wedge x_{2}\right)\left(y_{1} \wedge y_{2}\right)
$$

We also note that for any fixed $0<x_{0}<\infty$ the process $\left\{x_{0}^{-1 / 2} W\left(x_{0}, y\right), 0 \leq\right.$ $y<\infty\}$ is a (one-parameter) Wiener process and the same can be said about $\left\{y_{0}^{-1 / 2} W\left(x, y_{0}\right), 0 \leq x<\infty\right\}$.

Csörgö and Révész (1981) gave a constructive proof for the existence of a twoparameter Wiener process, and they also gave a detailed discussion about its law of the iterated logarithm. its increments, continuity modulus and some related Strassen-type functional limit theorems. In Chapter 5, we will present, discuss and prove various types of functional limit theorems for a two-parameter Wiener process.

A stochastic process $\{K(x, y), 0 \leq x \leq 1,0 \leq y \leq \infty\}$ is called a Kiefer process if it is a Gaussian process with zero mean and covariance function

$$
E K\left(x_{1}, y_{1}\right) K\left(x_{2}, y_{2}\right)=\left(x_{1} \wedge x_{2}-x_{1} x_{2}\right)\left(y_{1} \wedge y_{2}\right)
$$

for all $0 \leq x_{1}, x_{2} \leq 1$ and $0 \leq y_{1}, y_{2}<\infty$. It is well known to realize a Kiefer process by letting

$$
K(x, y)=W(x, y)-x W(1, y)
$$

for all $0 \leq x \leq 1$ and $0 \leq y<\infty$.

A stochastic process $\{\Gamma(x, y): 0 \leq x, y \leq 1\}$ is called a "tied down" Kiefer process 
if it is a Gaussian process with zero mean and covariance function

$$
E \Gamma\left(x_{1}, x_{2}\right) \Gamma\left(y_{1}, y_{2}\right)=\left(x_{1} \wedge x_{2}-x_{1} x_{2}\right)\left(y_{1} \wedge y_{2}-y_{1} y_{2}\right) .
$$

We can realize a "tied down" Kiefer process by letting

$$
\Gamma(x, y)=K(x, y)-y K(x, 1)
$$

for any $0 \leq x, y \leq 1$

Motivated by a study on change-point analysis (cf. Section 6.1), in Chapter 6 , we give a detailed discussion of large deviations in weighted metric for these three two-parameter Gaussian processes.

Finally, in Chapter 7. we study Brownian local time processes. For any Borel set $A$ of the real line the random measure

$$
H(A, t)=\lambda\{s: 0 \leq s \leq t, W(s) \in A\},
$$

where $\lambda$ is the Lebesgue measure, is called the occupation time of $W$. It is well known that $H(A, t)$ is absolutely continuous with respect to $\lambda$. The Radon-Nikodym derivative of $H$ is called the local time of $W$ and it will be denoted by $L$, i.e., $\{L(x, t),-\infty<x<\infty, 0 \leq t<\infty\}$ is defined by

$$
H(A, t)=\int_{A} L(x, t) d x .
$$

Trotter (1958) proved that $L(x, t)$ is continuous in both arguments. H. P. McKean Jr. (1962), D. B. Ray (1963), Hawkes (1971) and Perkins (1981) also discussed 
its modulus of continuity. Kesten (196.5) proved the law of the iterated logarithm for $L(0, t)$ and $\sup _{-\infty<x<\infty} L(x, t)$. Csáki. Csörgö. Földes and Révész (1983) proved large increment results for $L(x, t)$ which are similar to those Csörgő and Révész (1979) proved for a Wiener process (cf. also Chapter 3 of this thesis). In a similar vein, Chen $(1989,1990$ and 1991) studied some further fine analytic properties of $L(x, t)$, as indicated by the respective titles of these papers (cf. Bibliography), which will not be a part of this thesis. On the other hand. Chen (1997) constitutes the main part of Chapter 7 .

Motivated by Hanson and Russo (1983) and Chen, Kong and Lin (1986), we first discuss increments of the Brownian local time process and prove some results similar to those obtained by them for a Wiener process. Some large deviations for $L(x, t)$ and $\sup _{-\infty<x<\infty} L(x, t)$ are presented and proved. Finally, we establish Strassen type laws for both large time and small time increments.

As we have just mentioned, Chapter 7 is based on Chen (1997). The latter, as well as results that are covered in Chapters 2-6 of this thesis are believed to be new. In particular, Chapter 5 is based on the forthcoming paper, Chen (1998). Our results in Chapters 2, 3, 4 and 6 have not yet been published elsewhere. 


\section{Chapter 2}

\section{A Functional Modulus of Continuity for Wiener Processes}

\subsection{A Modulus of Continuity for a Wiener Process}

Let $\{W(t), 0 \leq t<\infty\}$ be a standard Wiener process. The following results are called its moduli of continuity.

Theorem 2.1.1 (P. Lévy 1937, 1948) We have

$$
\lim _{h \rightarrow 0} \sup _{0 \leq t \leq 1-h} \sup _{0<s \leq h} \frac{|W(t+s)-W(t)|}{\sqrt{2 h \log \frac{1}{h}}}=1 \quad \text { a.s. }
$$

and

$$
\lim _{h \rightarrow 0} \sup _{0 \leq t \leq 1-h} \frac{|W(t+h)-W(t)|}{\sqrt{2 h \log \frac{1}{h}}}=1 \quad \text { a.s. }
$$

In this chapter, we will combine Strassen's idea with Lévy's and obtain functional moduli of continuity, which also imply Theorem 2.1.1. Firstly, we obtain some large deviations in Section 2.2, and then present, discuss and prove our main results in Section 2.3 . 


\subsection{Large Deviations}

Let $C_{0}[0.1]$ be the space of continuous functions on the closed interval $[0,1]$ which vanish at the origin. For any $\varepsilon>0$, let $P_{\varepsilon}$ be probability measure on $C_{0}[0,1]^{2}$ corresponding to $W:=\{\sqrt{\varepsilon} W(t), 0 \leq t \leq 1\}$. Define the mapping $I: C_{0}[0,1] \rightarrow$ $[0, \infty]$ by

$$
I(f)= \begin{cases}\frac{1}{2} \int_{0}^{1}\left(f^{\prime}(x)\right)^{2} d x, & \text { if } f \text { is an absolutely continuous function } \\ & \text { with a square integrable } f^{\prime}(x) \\ \infty, & \text { otherwise. }\end{cases}
$$

Then, it is easy to show that $I(f)$ is lower semi-continuous and $\{f: I(f) \leq a\}$ is compact for any fixed $a>0$. Particularly, we have the following large deviation result:

Theorem 2.2.1 (M. Schilder 1966) For any closed set. $F \subset C_{0}[0,1]^{2}$, we have

$$
\limsup _{\varepsilon \rightarrow 0} \varepsilon \log P_{\varepsilon}(F) \leq-i n f_{f \in F} I(f)
$$

and. for any open set $G \subset C_{0}[0,1]^{2}$, we have

$$
\liminf _{\varepsilon \rightarrow 0} \varepsilon \log P_{\varepsilon}(G) \geq-i n f_{f \in G} I(f)
$$

Remark 2.2.1 If we replace $C_{0}[0,1]$ by $C_{0}[0, a](a>0)$, then the result corresponding to Theorem 2.2 .1 is also true.

We establish a large deviation for increments of Wiener processes as follows. 
Theorem 2.2.2 For any closed set $F \subset C_{0}[0,1]$ the inequality

$$
\underset{\varepsilon \rightarrow 0}{\limsup } \varepsilon\left(\log P\left(\cup_{0 \leq t \leq 1-h}\left\{\sqrt{\frac{\varepsilon}{h}}(W(t+h \cdot)-W(t)) \in F\right\}\right)+\log h\right) \leq-\inf _{f \in F} I(f)
$$

holds for every positive $h<1$.

For proving Theorem 2.2.2, we have the following lemma

Lemma 2.2.1 (Lemma 1.1.1, Csörgö-Révész 1981). For any $\mu>0$ there exists a constant $C=C(\mu)>0$ such that the inequality

$$
P\left(\sup _{0 \leq t \leq 1-h} \sup _{0<s \leq h}|W(t+s)-W(t)| \geq v \sqrt{h}\right) \leq \frac{C}{h} \exp \left\{-\frac{v^{2}}{2+\mu}\right\}
$$

holds for every positive $v$ and $0<h<1$.

Proof of Theorem 2.2.2 For any $0 \leq t \leq 1$, we have

$$
t=\sum_{k=0}^{\infty} \frac{\varepsilon_{k}(t)}{2^{k}}, \quad \varepsilon_{k}(t)=0,1 ; k=0,1,2, \cdots
$$

Set

$$
t_{n}:=\sum_{j=0}^{n} \frac{\varepsilon_{j}(t)}{2^{j}}, \quad n=0,1,2, \cdots
$$

For any $\delta>0$, let

$$
F^{\delta}=\left\{g \in C_{0}[0,1]: \inf _{f \in F}\|f-g\|<\delta\right\} .
$$

Then, for any positive integer $r$, we have

$$
\begin{aligned}
P\left(\cup _ { 0 \leq t \leq 1 - h } \left\{\sqrt{\frac{\varepsilon}{h}}(\right.\right. & W(t+h \cdot)-W(t)) \in F\}) \\
& \leq P\left(\cup_{0 \leq t \leq 1-h}\left\{\sqrt{\frac{\varepsilon}{h}}\left(W\left(t_{r}+h \cdot\right)-W\left(t_{r}\right)\right) \in F^{\delta}\right\}\right) \\
& +P\left(\cup_{0 \leq t \leq 1-h}\left\{\sqrt{\frac{\varepsilon}{h}}\left\|W(t+h \cdot)-W\left(t_{r}+h \cdot\right)\right\| \geq \frac{\delta}{2}\right\}\right) \\
:= & A+B .
\end{aligned}
$$


Take $r$ such that $2^{r+1}>K / h \geq 2^{r}$, where $K$ is a positive constant and will be specified later on. Then

$$
\begin{aligned}
A & \leq 2^{r} P\left(\sqrt{\varepsilon} W \in F^{\delta}\right) \\
& \leq \frac{K}{h} P\left(\sqrt{\varepsilon} W \in F^{\delta}\right) .
\end{aligned}
$$

Hence, by Theorem 2.2.1, we obtain

$$
\limsup _{\Sigma \rightarrow 0} \varepsilon(\log A+\log h) \leq-\inf _{f \in F^{2 \delta}} I(f) .
$$

Let $D:=\frac{1}{2} \sum_{j=0}^{\infty}\left(\sqrt{\frac{j}{2^{j}}}+\frac{1}{\sqrt{2^{j}}}\right)$. and $x_{j}:=\frac{1}{D} \sqrt{\frac{\xi \epsilon+1}{2^{j}}}$. For any $\mu>0$, by Lemma 2.2.1. we have

$$
\begin{aligned}
B & \leq P\left(\cup_{0 \leq t \leq 1-h}\left\{\sum_{j=0}^{\infty} \sqrt{\frac{\delta}{h}} \sup _{0 \leq x \leq 1}\left|W\left(t_{r+j+1}+h x\right)-W\left(t_{r+j}+h x\right)\right| \geq \delta \sum_{j+0}^{\infty} x_{j}\right\}\right) \\
& \leq \sum_{j=0}^{\infty} P\left(\cup_{0 \leq t \leq 1-h}\left\{\sqrt{\varepsilon} \sup _{0 \leq x \leq 1}\left|W\left(\frac{t_{r+j+1}}{h}+x\right)-W\left(\frac{t_{r+j}}{h}+x\right)\right| \geq \delta x_{j}\right\}\right) \\
& \leq C \sum_{j=0}^{\infty} 2^{2(r+j+1)} h \exp \left\{-(1-\mu) \delta^{2} 2^{r+j+1} h x_{j}^{2} / \varepsilon\right\} \\
& \leq 4 C K^{2} \frac{1}{h} \sum_{j=0}^{\infty} 2^{j} \exp \left\{-(1-\mu) \delta K^{\frac{j \varepsilon+1}{D^{2} \varepsilon}}\right\} \\
& =4 C K^{2} \frac{1}{h} \exp \left\{-(1-\mu) \delta K / D^{2} \varepsilon\right\} \sum_{j=0}^{\infty} 2^{j} \exp \left\{-\frac{(1-\mu) \delta K}{D^{2}} j\right\} \\
& \leq 4 C K^{2} E_{\frac{1}{h}} \exp \left\{-\frac{(1-\mu) \delta K}{D^{2}} \cdot \frac{1}{\varepsilon}\right\},
\end{aligned}
$$

where $E:=\sum_{j=0}^{\infty} 2^{j} \exp \left\{-\frac{(1-\mu) \delta K}{D^{2}} j\right\}$. Thus, for $K$ large enough, we obtain

$$
\limsup _{\varepsilon \rightarrow 0} \varepsilon(\log B+\log h) \leq-\frac{(1-\mu) \delta K}{D^{2}}
$$

and letting $\mu \rightarrow 0$, we get

$$
\limsup _{\varepsilon \rightarrow 0} \varepsilon(\log B+\log h) \leq-\frac{\delta K}{D^{2}} .
$$

Combining (2.2.5) and (2.2.6), for $K$ large enough, we obtain $\lim \sup _{\varepsilon \rightarrow 0} \varepsilon(\log (A+B)+\log h) \leq\left(\lim \sup _{\varepsilon \rightarrow 0} \varepsilon(\log (A h))\right) \vee\left(\lim \sup _{\varepsilon \rightarrow 0} \varepsilon(\log (A h))\right)$

$$
\begin{aligned}
& \leq\left(-\inf _{f \in F^{2 \delta}} I(f)\right) \vee\left(-\frac{\delta K}{D^{2}}\right) \\
& \leq-\inf _{f \in F^{2 \delta}} I(f) .
\end{aligned}
$$


Noting that $F$ is a closed set. it is easy to show that

$$
\lim _{\delta\llcorner 0} \inf _{f \in F^{2 \delta}} I(f)=\inf _{f \in F} I(f)
$$

Therefore the proof of Theorem 2.2.2 is complete.

\subsection{A Functional Modulus of Continuity}

We start with introducing a new functional limit theorem, a functional modulus of continuity for a Wiener process. For any $h \in(0,1)$ and $t \in[0,1-h]$, let

$$
M_{t, h}(x)=\frac{W(t+h x)-W(t)}{\sqrt{2 h \log \frac{1}{h}}}, \quad 0 \leq x \leq 1
$$

Define the sets $V_{h}$ and $S$ as follows:

$$
\begin{aligned}
& V_{h}=\left\{M_{t, h}(\cdot) \in C_{0}[0,1]: 0 \leq t \leq 1-h\right\}, \\
& S=\left\{f(\cdot) \in C_{0}[0,1]: I(f) \leq \frac{1}{2}\right\},
\end{aligned}
$$

where $I(\cdot)$ is defined in the Section 2.2 .

Theorem 2.3.1 For any $\varepsilon>0$, with probability one, there exists a $T_{0}=T_{0}(\varepsilon)$ such that

$$
V_{h} \subset S^{\varepsilon}
$$

and

$$
S \subset V_{h}^{\varepsilon}
$$

if $T \geq \mathcal{T}_{0}$, where the following notation is used:

$$
E^{\varepsilon}:=\left\{g \in C_{0}[0,1]: \inf _{f \in E} \sup _{o \leq x \leq 1}|g(x)-f(x)|<\varepsilon\right\}
$$

An equivalent result is the following theorem. 
Theorem 2.3.2 We have

$$
\lim _{h \rightarrow 0} \sup _{0 \leq t \leq 1-h} \inf _{f \in S} \sup _{0 \leq x \leq 1}\left|M_{t, h}(x)-f(x)\right|=0, \quad \text { a.s. }
$$

and for any $f \in S$

$$
\lim _{h \rightarrow 0} \inf _{0 \leq t \leq 1-h} \sup _{0 \leq x \leq 1}\left|M_{t . h}(x)-f(x)\right|=0, \quad \text { a.s. }
$$

Corollary 2.3.1 (2.3.3) and (2.3.4) imply (2.1.1) and (2.1.2).

From Corollary 2.3.1 we can see that the important difference between Theorem 2.3.1 (or Theorem 2.3.2) and the classic Strassen-type theorems is the fact that Theorem 2.3.1 implies "lim" results while classic Strassen-type theorems only imply "limsup" results. The key reason for this is (2.3.4), which in classic Strassen-type theorems has a weaker version as follows:

$$
\liminf _{h \rightarrow 0} \inf _{0 \leq t \leq 1-h} \sup _{0 \leq x \leq 1}\left|M_{t, h}(x)-f(x)\right|=0, \quad \text { a.s. }
$$

We note in passing that, in the same spirit, via (2.3.4), Csörgő and Révész (1981) succeeded in replacing "limsup" in the original P. Lévy moduli by "lim" as in Theorem 2.1.1.

Proof of Theorem 2.3.2 For any $\theta>1$ and integer $n \geq 1$, let $h_{n}=\theta^{-n}$. First of all, we show that

$$
\lim _{n \rightarrow \infty} \sup _{0 \leq t \leq 1-h_{n+1}} \inf _{f \in S} \sup _{0 \leq x \leq 1}\left|M_{t, h_{n}}(x)-f(x)\right|=0, \quad \text { a.s. }
$$


For any $\varepsilon>0$ and $\mu>0$, by Theorem 2.2.2, we have

$$
\begin{aligned}
& P\left(\sup _{0 \leq t \leq 1-h_{n+1}} \inf _{f} \leq S \sup _{0 \leq x \leq 1}\left|M_{t, h_{n}}(x)-f(x)\right| \geq \varepsilon\right) \\
& \leq P\left(\cup_{0 \leq t \leq 1-h_{n+1}}\left\{M_{t . h_{n}}(\cdot) \in\left(S^{\Xi}\right)^{c}\right\}\right) \\
& \leq P\left(\cup_{0 \leq t \leq 1-h_{n}}\left\{M_{t . h_{n}}(\cdot) \in\left(S^{\varsigma}\right)^{c}\right\}\right) \\
&+P\left(\cup_{1-h_{n} \leq t \leq 1-h_{n+1}}\left\{M_{t . h_{n}}(\cdot) \in\left(S^{\varepsilon}\right)^{c}\right\}\right) \\
& \leq 2 P\left(\cup_{0 \leq t \leq 1-h_{n}}\left\{M_{t, h_{n}}(\cdot) \in\left(S^{\varsigma}\right)^{c}\right\}\right) \\
& \leq \frac{2}{h_{n}} \exp \left\{-2(1-\mu) \inf _{f \in\left(S^{c}\right)^{c}} I(f) \log \frac{1}{h_{n}}\right\} \\
&=2 \theta^{\left.2(1-\mu) \inf _{f \in\left(S^{c}\right)^{c}} I(f)-1\right) n}
\end{aligned}
$$

if $n$ is large enough. Take $\mu$ small enough such that.

$$
\eta:=2(1-\mu) \inf _{f \in\left(S^{*}\right)^{c}} I(f)>1
$$

Hence we obtain

$$
\sum_{n} P\left(\sup _{0 \leq t \leq 1-h_{n+1}} \inf _{f \in S} \sup _{0 \leq x \leq 1}\left|M_{t, h_{n}}(x)-f(x)\right| \geq \varepsilon\right)<\infty,
$$

which, by the Borel-Cantelli lemma, implies (2.3.5).

For all $h \in(0,1)$, there exists $n$ such that $h_{n+1} \leq h<h_{n}$. Then we have

$$
\begin{aligned}
& \sup _{0 \leq t \leq 1-h} \inf _{f \in S} \sup _{0 \leq x \leq 1}\left|M_{t, h}(x)-f(x)\right| \\
& \quad \leq \sup _{0 \leq t \leq 1-h_{n+1}} \inf _{f \in S} \sup _{0 \leq x \leq 1}\left|M_{t, h_{n}}\left(\frac{h}{h_{n}} x\right)-f\left(\frac{h}{h_{n}} x\right)\right| \\
& \quad+\sup _{0 \leq t \leq 1-h_{n+1}} \sup _{0 \leq x \leq 1}\left(\sqrt{\frac{h_{n} \log \frac{1}{h_{n}}}{h \log \frac{1}{h}}}-1\right)\left|\frac{W\left(t+h_{n} \frac{h}{h_{n}} x\right)-W(t)}{\sqrt{2 h_{n} \log \frac{1}{h_{n}}}}\right| \\
&+\sup _{f \in S} \sup _{0 \leq x \leq 1}\left|f\left(\frac{h}{h_{n}} x\right)-f(x)\right| \\
&:=I_{1}+I_{2}+I_{3} .
\end{aligned}
$$

Via (2.3.7), it is obtained that

$$
\lim _{n \rightarrow \infty} I_{1}=0 \quad \text { a.s. }
$$


and for $n$ large enough we have

$$
\sup _{0 \leq t \leq 1-h_{n+1}} \sup _{0 \leq x \leq 1}\left|\frac{W\left(t+h_{n} \frac{h}{h_{n}} x\right)-W(t)}{\sqrt{2 h_{n} \log \frac{1}{h_{n}}}}\right| \leq 2 \quad \text { a.s. }
$$

Since

$$
\sqrt{\frac{h_{n} \log \frac{1}{h_{n}}}{h \log \frac{1}{h}}}-1 \leq \sqrt{\theta}-1
$$

thus

$$
\lim _{n \rightarrow \infty} I_{2} \leq \sqrt{\theta}-1 \quad \text { a.s. }
$$

Concerning $I_{3}$, we have

$$
I_{3} \leq \sup _{f \in S} \sup _{0 \leq x \leq 1}\left(1-\frac{h_{n+1}}{h_{n}}\right) x \int_{0}^{1}\left(f^{\prime}(u)\right)^{2} d u \leq \theta-1 .
$$

Combining (2.3.6), (2.3.8) and (2.3.9), we obtain

$$
\lim _{h \rightarrow 0} \sup _{0 \leq t \leq 1-h} \inf _{f \in S} \sup _{0 \leq x \leq 1}\left|M_{t, h}(x)-f(x)\right| \leq 2(\theta-1) \quad \text { a.s.. }
$$

Since we can take any $\theta>1$, therefore (2.3.3) is true.

Next, we show that (2.3.4) holds for any $f \in S$. For all $h \in(0,1)$, there exists $n$ iuch that $h_{n+1} \leq h<h_{n}$. Then, for any $f \in S$, we have

$$
\begin{aligned}
& \inf _{0 \leq t \leq 1-h} \sup _{0 \leq x \leq 1}\left|M_{t, h}(x)-f(x)\right| \\
& \quad \leq \inf _{0 \leq t \leq 1-h_{n}} \sup _{0 \leq x \leq 1}\left|M_{t, h_{n}}\left(\frac{h}{h_{n}} x\right)-f\left(\frac{h}{h_{n}} x\right)\right| \\
&+\sup _{0 \leq t \leq 1-h_{n+1}} \sup _{0 \leq x \leq 1}\left(\sqrt{\frac{h_{n} \log \frac{l}{h_{n}}}{h \log \frac{1}{h}}}-1\right)\left|\frac{W\left(t+h_{n} \frac{h}{h_{n}} x\right)-W(t)}{\sqrt{2 h_{n} \log \frac{1}{h_{n}}}}\right| \\
&+\sup _{f \in S} \sup _{0 \leq x \leq 1}\left|f\left(\frac{h}{h_{n}} x\right)-f(x)\right| \\
&:=I_{4}+I_{2}+I_{3} .
\end{aligned}
$$


For any $\varepsilon>0$ and $\mu>0$. by Theorem 2.2 .1 , we have

$$
\begin{aligned}
& P\left(\inf _{0 \leq r \leq 1-h_{n}} \sup _{0 \leq x \leq 1}\left|M_{t, h_{n}}(x)-f(x)\right| \geq \varepsilon\right) \\
& \leq P\left(\inf _{0 \leq i \leq\left[\frac{1}{h_{n}}\right]-1} \sup _{0 \leq x \leq 1}\left|M_{i h_{n}, h_{n}}(x)-f(x)\right| \geq \varepsilon\right) \\
& \leq\left(P\left(\sup _{0 \leq x \leq 1}\left|\frac{W(x)}{\sqrt{2 \log \frac{1}{h_{n}}}}-f(x)\right| \geq \varepsilon\right)\right)^{\left[\frac{1}{h_{n}} \mid-1\right.} \\
& \leq\left\{1-P\left(\frac{1}{\sqrt{2 \log \frac{1}{h_{n}}}} W \in B(f, \varepsilon)\right)\right\}^{\left\{\frac{1}{h_{n}} \mid-1\right.} \\
& \leq\left(1-\exp \left\{-2(1+\mu) \inf _{g \in B(f, \varepsilon)} I(g) \log \frac{1}{h_{n}}\right\}\right)^{\left[\frac{1}{h_{n}} \mid-1\right.} \\
& \leq 2 e^{-\theta^{\left(1-2(1+\mu) \inf _{g \in B(f, \varepsilon)} I(g)\right)_{n}}}
\end{aligned}
$$

if $n$ is large enough. Since $\inf _{g \in B(f, \varepsilon)} I(g)<\frac{1}{2}$, taking $\mu$ small enough. we have

$$
1-2(1+\mu) \inf _{g \in B(f, \varepsilon)} I(g)>0
$$

Hence

$$
\sum_{n} P\left(\inf _{0 \leq t \leq 1-h_{n}} \sup _{0 \leq x \leq 1}\left|M_{t, h_{n}}(x)-f(x)\right| \geq \varepsilon\right)<\infty .
$$

Therefore, by the Borel-Cantelli lemma, we obtain

$$
\lim _{n \rightarrow \infty} \inf _{0 \leq t \leq 1-h} \sup _{0 \leq x \leq 1}\left|M_{t, h}(x)-f(x)\right|=0 \quad \text { a.s. }
$$

which implies that

$$
\lim _{n \rightarrow \infty} I_{4}=0 \quad \text { a.s.. }
$$

Combining (2.3.6), (2.3.8) and (2.3.10), we obtain

$$
\lim _{h \rightarrow 0} \inf _{0 \leq t \leq 1-h} \sup _{0 \leq x \leq 1}\left|M_{t, h}(x)-f(x)\right| \leq 2(\theta-1) \quad \text { a.s. }
$$

for any $f \in S$. Since we can take any $\theta>1$, therefore (2.3.4) is true. The proof of Theorem 2.3.2 is complete. 
Proof of Corollary 2.3.1 Firstly: using (2.3.3), we have

$$
\begin{aligned}
& \lim \sup _{h \rightarrow 0} \sup _{0 \leq t \leq 1-h} \sup _{0 \leq s \leq h} \frac{|W(t+s)-W(t)|}{\sqrt{2 h \log \frac{h}{h}}} \\
& \quad \leq \limsup _{h \rightarrow 0} \sup _{0 \leq t \leq 1-h} \inf _{f \in S} \sup _{0 \leq x \leq 1}\left|\frac{W(t+h x)-W(t)}{\sqrt{2 h \log \frac{1}{h}}}-f(x)\right| \\
&+\limsup _{h \rightarrow 0} \sup _{f \in S} \sup _{0 \leq x \leq 1}|f(x)| \\
& \leq \sup _{f \in S} \int_{0}^{1}\left(f^{\prime}(t)\right)^{2} d t=1 .
\end{aligned}
$$

Next, by (2.3.4), for any $f \in S$ we also have

$$
\begin{aligned}
& \lim \inf _{h \rightarrow 0} \sup _{0 \leq t \leq 1-h} \frac{|W(t+h)-W(t)|}{\sqrt{2 h \log \frac{1}{h}}} \\
& \quad=\liminf _{h \rightarrow 0} \sup _{0 \leq t \leq 1-h}\left|\frac{W(t+h)-W(t)}{\sqrt{2 h \log \frac{1}{h}}}-f(1)+f(1)\right| \\
& \geq|f(1)|-\limsup \sin _{h \rightarrow 0} \inf _{0 \leq t \leq 1-h} \sup _{0 \leq x \leq 1}\left|\frac{W(t+h)-W(t)}{\sqrt{2 h \log \frac{1}{h}}}-f(x)\right| \\
&=|f(1)| .
\end{aligned}
$$

Hence

$$
\liminf _{h \rightarrow 0} \sup _{0 \leq t \leq 1-h} \frac{|W(t+h)-W(t)|}{\sqrt{2 h \log \frac{1}{h}}} \geq \sup _{f \in S}|f(1)|=1 .
$$

Therefore, we obtain (2.1.1) and (2.1.2), and the proof of Corollory 2.3.1 is complete.

\section{$2.4 \quad$ A Note}

Gantert, N. (1993) showed that with probability one

$$
\left\{\frac{W(h x)}{\sqrt{2 h \log \log \frac{1}{h}}}, 0 \leq x \leq 1,0<h<1\right\} \subset C_{0}[0,1]
$$

is relatively compact in $C_{0}[0,1]$, and the set of its limit points (as $h \rightarrow 0$ ) is $S$. Generally, we also have that with probability one

$$
\left\{\frac{W\left(t_{0}+h x\right)-W\left(t_{0}\right)}{\sqrt{2 h \log \log \frac{1}{h}}}, 0 \leq x \leq 1,0<h<1\right\} \subset C_{0}[0,1]
$$


is relatively compact in $C_{0}[0,1]$, and the set of its limit points (as $h \rightarrow 0$ ) is $S$ for any $t_{0} \in[0, \infty)$. This result implies that

$$
\limsup _{h \rightarrow 0} \frac{\left|W\left(t_{0}+h x\right)-W\left(t_{0}\right)\right|}{\sqrt{2 h \log \log \frac{1}{h}}}=1 \quad \text { a.s. }
$$

which implies that for any $t_{0} \in[0, \infty)$ a Wiener process $\{W(t), 0 \leq t<\infty\}$ is continuous. Hence we usually call (2.4.1) a local modulus of continuity theorem. It is interesting to compare (2.4.1) with (2.1.1) or (2.1.2), which in this light can be called uniform modulus of continuity theorems. 


\section{Chapter 3}

\section{Csörgö-Révész Increments}

\subsection{Csörgö-Révész Increments of a Wiener Pro- cess}

Let $\{W(t), 0 \leq t<\infty\}$ be a standard Wiener process, and let $a_{T}$ be a positive function of $T \geq 3$. Introduce the following notations:

$$
\begin{aligned}
& \beta_{T}=\left(2 a_{T}\left(\log T a_{T}^{-1}+\log \log T\right)\right)^{-\frac{1}{2}} ; \\
& Y_{t, T}(x)=\beta_{T}\left(W\left(t+x a_{T}\right)-W(t)\right), \quad 0 \leq x \leq 1, \quad 0 \leq t \leq T-a_{T} .
\end{aligned}
$$

Csörgö, M. and Révész, P. established the following well-known results.

Theorem 3.1.1 (Csörgö, M. and Révész, P. 1979 and 1981) Let $0<a_{T} \leq T$ satisfy the following conditions:

(i) $a_{T}$ is non-decreasing

(ii) $T a_{T}^{-1}$ is non-decreasing.

Then we have

$$
\limsup _{T \rightarrow \infty} Y_{T-a_{T}, T}(1)=\limsup _{T \rightarrow \infty} \sup _{0 \leq t \leq T-a_{T}} \sup _{0 \leq x \leq 1}\left|Y_{t, T}(x)\right|=1 \quad \text { a.s. }
$$

If we also assume that 
(iii) $\lim _{T \rightarrow \infty} \frac{\log T a_{T}^{-1}}{\log \log T}=\infty$,

then we also have

$$
\lim _{T \rightarrow \infty} \sup _{0 \leq t \leq T-a_{T}} Y_{t, T}(1)=\lim _{T \rightarrow \infty} \sup _{0 \leq t \leq T-a_{T}} \sup _{0 \leq x \leq 1}\left|Y_{t . T}(x)\right|=1 \quad \text { a.s. }
$$

Remark 3.1.1 Taking $a_{T}=T$, we get the classic law of the iterated logarithm from (3.1.1).

Recall the definitions of $I(f)$ and $S$ (cf. Chapter 2):

$$
I(f)= \begin{cases}\frac{1}{2} \int_{0}^{1}\left(f^{\prime}(x)\right)^{2} d x, & \text { if } f \text { is an absolutely continuous function } \\ & \text { with a square integrable } f^{\prime}(x) \\ \infty, & \text { otherwise }\end{cases}
$$

and

$$
S=\left\{f(\cdot) \in C_{0}[0,1]: I(f) \leq \frac{1}{2}\right\} .
$$

Chan, A.H.C., Csörgö, M. and Révész, P. (1978) combined the above result with Strassen's functional law of the iterated logarithm, and obtained the following theorem.

Theorem 3.1.2 If $0<a_{T} \leq T$ satisfies conditions (i) and (ii), then, with probability one, $\left\{Y_{T-a_{T} \cdot T}(x): 0 \leq x \leq 1, T \geq 3\right\}$ (as $T \rightarrow \infty$ ) is relatively compact in $C_{0}[0,1]$, and the set of its limit points is $S$.

Remark 3.1.2 Taking $x=1$ in Theorem 3.1.2 and noting that

$$
\sup _{f \in S} f(1)=\sup _{f \in S} \sup _{0 \leq x \leq 1}|f(x)|=1,
$$

it is easy to see that Theorem 3.1.2 implies

$$
\limsup _{T \rightarrow \infty} Y_{T-a_{T}}(1)=\limsup _{T \rightarrow \infty} \sup _{0 \leq x \leq 1}\left|Y_{T-a_{T}}(x)\right|=1 \quad \text { a.s. }
$$


Révész. P. (1979) also combined Theorem 3.1.1 with Strassen's theorem. and obtained the following theorem.

Theorem 3.1.3 Assume that $0<a_{T} \leq T$ satisfies conditions (i) and (ii). Then, with probability one., $\left\{Y_{t . T}(x): 0 \leq x \leq 1,0 \leq t \leq T-a_{T} . T \geq 3\right\}$ (as $T \rightarrow \infty$ ) is relatively compact in $C_{0}[0,1]$, and the set of its limit points is $S$. If we also assume that $a_{T}$ satisfies condition (iii), then we also have that

$$
\lim _{T \rightarrow \infty} \sup _{0 \leq t \leq T-a_{T}} \inf _{f \in S} \sup _{0 \leq x \leq 1}\left|Y_{t, T}(x)-f(x)\right|=0 \quad \text { a.s. },
$$

and for any $f \in S$

$$
\lim _{T \rightarrow \infty} \inf _{T \leq t \leq T-a_{T}} \sup _{0 \leq x \leq 1}\left|Y_{t, T}(x)-f(x)\right|=0 \quad \text { a.s. }
$$

Corollary 3.1.1 (3.1.3) and (3.1.4) imply (3.1.2).

Proof of Corollary 3.1.1 Firstly, by (3.1.3), we have

$$
\begin{aligned}
& \lim \sup _{T \rightarrow \infty} \sup _{0 \leq t \leq T-a_{T}} \sup _{0 \leq x \leq 1}\left|Y_{t, T}(x)\right| \\
& \quad \leq \lim \sup _{T-\infty} \sup _{0 \leq t \leq T-a_{T}} \inf _{f \in S} \sup _{0 \leq x \leq 1}\left|Y_{t, T}(x)-f(x)\right|+\sup _{f \in S} \sup _{0 \leq x \leq 1}|f(x)| \\
& \quad=\sup _{f \in S} \sup _{0 \leq x \leq 1}|f(x)|=1 \text { a.s. }
\end{aligned}
$$

Also, via (3.1.4), for any $f \in S$

$$
\begin{aligned}
& \lim \inf _{T \rightarrow \infty} \sup _{0 \leq t \leq T-a_{T}} Y_{t, T}(1) \\
& \quad \geq f(1)-\lim _{T \rightarrow \infty} \inf _{0 \leq t \leq T-a_{T}} \sup _{0 \leq x \leq 1}\left|Y_{t, T}(x)-f(x)\right| \\
& \quad=f(1) \quad \text { a.s. }
\end{aligned}
$$

Hence

$$
\liminf _{T \rightarrow \infty} \sup _{0 \leq t \leq T-a_{T}} Y_{t, T}(1) \geq \sup _{f \in S} f(1)=1 \quad \text { a.s. }
$$

Therefore, the proof is now complete. 
An interesting question is: what happens if condition (iii) is replaced by

$$
\lim _{T \rightarrow \infty} \frac{\log T a_{T}^{-1}}{\log \log T}=r, \quad(0 \leq r \leq \infty) ?
$$

We answer this question in the next section.

\subsection{Functional Limit Theorems for Csörgö-Révész Increments of a Wiener Process}

In this section we generalize Theorem 3.1.3. Introduce the notation:

$$
S_{\alpha}=\left\{f \in C_{0}[0,1]: I(f) \leq \frac{\alpha^{2}}{2}\right\}, \quad \alpha \geq 0 .
$$

Let

$$
\alpha(r)= \begin{cases}\sqrt{\frac{r}{r+1}}, & 0 \leq r<\infty, \\ 1, & r=\infty .\end{cases}
$$

Theorem 3.2.1 Assume that $0<a_{T} \leq T$ satisfies conditions (i) and (ii). Then, with probability one, $\left\{Y_{t . T}(x): 0 \leq x \leq 1,0 \leq t \leq T-a_{T}, T \geq 3\right\}(a s T \rightarrow \infty)$ is relatively compact in $C_{0}[0,1]$, and the set of its limit points is $S$. If we also assume that $a_{T}$ satisfies the following condition:

$$
\text { (iv) } \lim _{T \rightarrow \infty} \frac{\log T a_{T}^{-1}}{\log \log T}=r, \quad(0 \leq r \leq \infty),
$$

then we also have that

$$
\liminf _{T \rightarrow \infty} \sup _{0 \leq t \leq T-a_{T}} \inf _{f \in S_{\alpha(r)}} \sup _{0 \leq x \leq 1}\left|Y_{t, T}(x)-f(x)\right|=0 \quad \text { a.s., }
$$

and for any $f \in S_{\alpha(r)}$

$$
\lim _{T \rightarrow \infty} \inf _{0 \leq t \leq T-a_{T}} \sup _{0 \leq x \leq 1}\left|Y_{t, T}(x)-f(x)\right|=0 \quad \text { a.s. }
$$


Remark 3.2.1 The proof of this theorem will show that it remains true if $T$ is running over all the integers.

Corollary 3.2.1 (Book, Shore 1978) Let $a_{T}$ be as in Theorem 3.2.1. Then

$$
\liminf _{T \rightarrow \infty} \sup _{0 \leq t \leq T-a_{T}} Y_{t . T}(1)=\liminf _{T \rightarrow \infty} \sup _{0 \leq t \leq T-a_{T}} \sup _{0 \leq x \leq 1}\left|Y_{t, T}(x)\right|=\alpha(r) \quad \text { a.s. }
$$

Proof of Theorem 3.2.1 According to Theorem 3.1.3, we only need to prove (3.2.1) and (3.2.2). For $n \geq 1$, take $T_{n}=e^{e^{n}}$. To show (3.2.1), it suffies to show that for any $\varepsilon>0$

$$
\limsup _{n \rightarrow \infty} \sup _{0 \leq t \leq T_{n}-a_{T_{n}}} \inf _{f \in S_{\mathbf{a}(r)}} \sup _{0 \leq x \leq 1}\left|Y_{t, T_{n}}(x)-f(x)\right|<\varepsilon \quad \text { a.s. }
$$

For any $\mu>0$, by Theorem 2.2.2. for $n$ large enough, we have

$$
\begin{aligned}
P\left(\sup _{0 \leq t \leq T_{n}-a_{T_{n}}} \inf _{f \in S_{\alpha(r)}} \sup _{0 \leq x \leq 1}\left|Y_{t, T_{n}}(x)-f(x)\right| \geq \varepsilon\right) \\
\quad \leq P\left(\cup_{0 \leq t \leq 1-\frac{a_{T_{n}}}{T_{n}}}\left\{\left(2 \frac{a_{T_{n}}}{T_{n}}\left(\log \frac{T_{n} \log T_{n}}{a_{T_{n}}}\right)\right)^{-\frac{1}{2}}\left(W\left(t+\frac{a_{T_{n}}}{T_{n}} \cdot\right)-W(t)\right) \in\left(\left(S_{\alpha(r)}\right)^{\varepsilon}\right)^{c}\right\}\right) \\
\leq \frac{T_{n}}{a_{T_{n}}} \exp \left\{-2(1-\mu) \inf _{f \in\left(\left(S_{\alpha(r)}\right)^{c}\right)^{c}} I(f) \log \frac{T_{n} \log T_{n}}{a_{T_{n}}}\right\} \\
\quad=\frac{T_{n}}{a_{T_{n}}}\left(\frac{a_{T_{n}}}{T_{n} \log T_{n}}\right)^{2(1-\mu) \inf _{f \in\left(\left(S_{\alpha(r)^{c}}\right)^{c} I(f)\right.}} .
\end{aligned}
$$

Taking $\mu$ small enough, there exists some $\delta>0$ such that

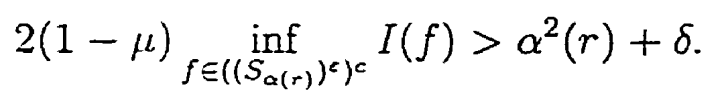

Hence

$$
P\left(\sup _{0 \leq t \leq T_{n}-a_{T_{n}}} \inf _{f \in S_{\alpha(r)}} \sup _{0 \leq x \leq 1}\left|Y_{t, T_{n}}(x)-f(x)\right| \geq \varepsilon\right) \leq\left(\frac{T_{n}}{a_{T_{n}}}\right)^{1-\alpha^{2}(r)-\delta}\left(\frac{1}{\log T_{n}}\right)^{\alpha^{2}(r)+\delta} .
$$

If $1-\alpha^{2}(r)-\delta<0$, then

$$
P\left(\sup _{0 \leq t \leq T_{n}-a_{T_{n}}} \inf _{f \in S_{\alpha(r)}} \sup _{0 \leq x \leq 1}\left|Y_{t, T_{n}}(x)-f(x)\right| \geq \varepsilon\right) \leq\left(\frac{1}{\log T_{n}}\right)^{\alpha^{2}(r)+\delta}=e^{-\left(\alpha^{2}(r)+\delta\right) n} .
$$


hence. by Theorem 2.2.1 and properties of a Wiener process. for $n$ large enough we have

$$
\begin{aligned}
& P\left(\inf _{0 \leq t \leq T_{n}-a_{T_{n}}} \sup _{0 \leq x \leq 1}\left|Y_{t, T_{n+1}}(x)-f(x)\right| \geq \varepsilon\right) \\
& \leq P\left(\min _{0 \leq j \leq\left\{\frac{T_{n}-a_{T_{n}}}{a_{T_{n+1}}}\right]} \sup _{0 \leq x \leq 1}\left|Y_{j a_{T_{n+1}} \cdot T_{n+1}}(x)-f(x)\right| \geq \varepsilon\right) \\
& =\left[P\left(\sup _{0 \leq x \leq 1}\left|Y_{0, T_{n+1}}(x)-f(x)\right| \geq \varepsilon\right)\right]^{\frac{T_{n}-a_{T_{n}}}{a_{T_{n+1}}}} \\
& \leq\left(1-e^{-2(1+\mu) \inf _{g \in B(f, c)} I(g) \log \frac{T_{n+1} \log T_{n+1}}{{ }^{a} T_{n+1}}}\right)^{\frac{T_{n}-a_{T_{n}}}{{ }^{a} T_{n+1}}}
\end{aligned}
$$

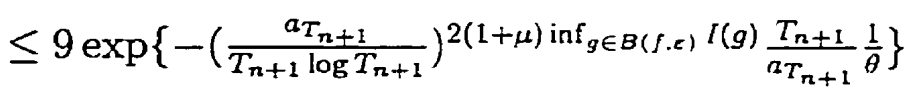

$$
\begin{aligned}
& \leq 9 \exp \left\{-\frac{1}{\theta}\left(\frac{T_{n+1}}{a_{T_{n+1}}}\right)^{1-\alpha^{2}(r)+\delta}\left(\frac{1}{\log T_{n+1}}\right)^{\alpha^{2}(r)-\delta}\right\} .
\end{aligned}
$$

Since, by condition (iv), for $n$ large enough

$$
\frac{T_{n+1}}{a_{T_{n+1}}}>\left(\log T_{n+1}\right)^{r-\mu} .
$$

we obtain

$$
\begin{aligned}
P\left(\inf _{0 \leq t \leq T_{n}-a_{T_{n}}} \sup _{0 \leq x \leq 1}\left|Y_{t, T_{n+1}}(x)-f(x)\right| \geq \varepsilon\right) \\
\leq 9 \exp \left\{-\frac{1}{\theta}\left(\frac{1}{\log T_{n+1}}\right)^{-(r-\mu)\left(1-\alpha^{2}(r)+\delta\right)+\alpha^{2}(r)-\delta}\right\} \\
\leq 9 \exp \left\{-\frac{1}{\theta}(n \log \theta)^{\delta(r+1) \alpha^{2}(r)+\delta-\mu\left(\delta+1-\alpha^{2}(r)\right)}\right\} .
\end{aligned}
$$

Hence, taking $\mu<\delta /(\delta+1)$, we obtain

$$
\sum_{n} P\left(\inf _{0 \leq t \leq \mathcal{T}_{n}-a_{T_{n}}} \sup _{0 \leq x \leq 1}\left|Y_{t, T_{n+1}}(x)-f(x)\right| \geq \varepsilon\right)<\infty .
$$

Therefore, by Borel-Cantelli lemma, we get that

$$
\limsup _{n \rightarrow \infty} \inf _{0 \leq t \leq T_{n}-a_{T_{n}}} \sup _{0 \leq x \leq 1}\left|Y_{t, T_{n+1}}(x)-f(x)\right|=0 \quad \text { a.s. }
$$

which implies that

$$
\lim _{n \rightarrow \infty} A_{n}=0 \quad \text { a.s. }
$$


Using $T_{n} a_{T_{n}}^{-1} \leq T_{n+1} a_{T_{n+1}}^{-1}$ and Theorem 3.1.3, it is easy to see

$$
\begin{aligned}
\limsup _{n \rightarrow \infty} B_{n} & \leq 2 \lim \sup _{n \rightarrow \infty}\left\{\left(\frac{T_{n+1}}{T_{n}} \frac{\left.\left.\frac{\log \frac{T_{n+1} \log T_{n+1}}{a} T_{n+1}}{\log \frac{T_{n} \log T_{n}}{a_{n+1}}}\right)^{\frac{1}{2}}-1\right\}}{}\right.\right. \\
& \leq 2 \lim \sup _{n \rightarrow \infty}\left\{\sqrt{\theta}\left(\frac{\log \left(\frac{T_{n+1} \log T_{n+1}}{T_{n+1}} \frac{a_{T_{n}}}{T_{n} \log T_{n}}\right)}{\log \frac{T_{n} \log T_{n}}{a T_{n}}}+1\right)-1\right\} \\
& \leq 2 \lim \sup _{n \rightarrow \infty}\left\{\sqrt{\theta}\left(\frac{\log \theta+\log \frac{n+1}{n}}{\log n+\log \log \theta}+1\right)-1\right\} \\
& \leq 2(\sqrt{\theta}-1) \quad \text { a.s. }
\end{aligned}
$$

Letting $\theta \downarrow 1$. we obtain

$$
\lim _{n \rightarrow \infty} B_{n}=0 \quad \text { a.s. }
$$

By the definition of $S_{\alpha(r)}$ and $\frac{a_{T_{n}}}{a_{T_{n+1}}} \leq \frac{T_{n}}{T_{n+1}}=\frac{1}{\theta}$, we have

$$
\begin{aligned}
C_{n} & \leq \sup _{f \in S_{\alpha(r)}} \sup _{0 \leq x \leq 1}\left|\int_{\frac{a_{T}}{a_{T_{n+1}}} x}^{x} f^{\prime}(t) d t\right| \\
& \leq \sup _{f \in S_{\alpha(r)}} \sup _{0 \leq x \leq 1}\left(\int_{\frac{a_{T}}{a_{T_{n+1}}} x}^{x}\left(f^{\prime}(t)\right)^{2} d t\right)^{\frac{1}{2}}\left(1-\frac{a_{T_{n}}}{a_{T_{n+1}}}\right) x \\
& \leq\left(1-\frac{1}{\theta}\right) \alpha(r) .
\end{aligned}
$$

Now letting $\theta \downarrow 1$. we conclude

$$
\lim _{n \rightarrow \infty} C_{n}=0 \quad \text { a.s. }
$$

Therefore, combining (3.2.6), (3.2.7) and (3.2.8), we obtain (3.2.2). This also completes the proof of Theorem 3.2.1.

Proof of Corollary 3.2.1 Firstly, by (3.2.1), we have

$$
\begin{aligned}
& \lim \inf _{T \rightarrow \infty} \sup _{0 \leq t \leq T-a_{T}} \sup _{0 \leq x \leq 1}\left|Y_{t, T}(x)\right| \\
& \quad \leq \liminf \inf _{T-\infty} \sup _{0 \leq t \leq T-a_{T}} \inf _{f \in S_{\alpha(r)}} \sup _{0 \leq x \leq 1}\left|Y_{t, T}(x)-f(x)\right|+\sup _{f \in S_{\alpha(r)}} \sup _{0 \leq x \leq 1}|f(x)| \\
& \quad=\sup _{f \in S_{\alpha(r)}} \sup _{0 \leq x \leq 1}|f(x)|=\alpha(r) \quad \text { a.s. }
\end{aligned}
$$


Also, via (3.2.2). for any $f \in S_{\alpha(r)}$

$$
\begin{aligned}
& \lim \inf _{T \rightarrow \infty} \sup _{0 \leq t \leq T-a_{T}} Y_{t, T}(1) \\
& \quad \geq f(1)-\lim _{T \rightarrow \infty} \inf _{0 \leq t \leq T-a_{T}} \sup _{0 \leq x \leq 1}\left|Y_{t, T}(x)-f(x)\right| \\
& \quad=f(1) \quad \text { a.s. }
\end{aligned}
$$

Hence

$$
\liminf _{T \rightarrow \infty} \sup _{0 \leq t \leq T-\Omega_{T}} Y_{t, T}(1) \geq \sup _{f \in S_{\alpha(r)}} f(1)=\alpha(r) \quad \text { a.s. }
$$

This also completes the proof.

Remark 3.2.2 We note that Theorem 3.2.1 is not sharp for the case of $r=0$. This motivates to introduce the following problem: find the normalizing factor $\gamma_{T}$ instead of $\beta_{T}$ such that (3.2.1) and (3.2.2) hold true for $S$ instead of $S_{\alpha(r)}$. Actually, we have the following answer.

Theorem 3.2.2 Assume that $0<a_{T} \leq T$ satisfies conditions (i), (ii) and

(v) $\lim _{T \rightarrow \infty}\left(T / a_{T}\right) / \log \log T=\infty$

Then, we have that.

$$
\liminf _{T \rightarrow \infty} \sup _{0 \leq t \leq T-a_{T}} \inf _{f \in S} \sup _{0 \leq x \leq 1}\left|Z_{t, T}(x)-f(x)\right|=0 \quad \text { a.s., }
$$

and for any $f \in S$

$$
\lim _{T \rightarrow \infty 0 \leq t \leq T-a_{T}} \sup _{0 \leq x \leq 1}\left|Z_{t, T}(x)-f(x)\right|=0 \quad \text { a.s. }
$$

where

$$
\begin{aligned}
& Z_{t, T}(x)=\gamma_{T}\left(W\left(t+x a_{T}\right)-W(t)\right), \quad 0 \leq x \leq 1, \quad 0 \leq t \leq T-a_{T}, \\
& \gamma_{T}=\left(2 a_{T}\left(\log T a_{T}^{-1}-\log \log \log T\right)\right)^{-\frac{1}{2}} .
\end{aligned}
$$

Corollary 3.2.2 (Shao 1986) Under the conditions of Theorem 3.2.2, we have

$$
\liminf _{T \rightarrow \infty} \sup _{0 \leq t \leq T-a_{T}} Z_{t, T}(1)=\liminf _{T \rightarrow \infty} \sup _{0 \leq t \leq T-a_{T}} \sup _{0 \leq x \leq 1}\left|Z_{t, T}(x)\right|=1 \quad \text { a.s. }
$$


The proof of Corollary 3.2 .2 is similar to that of Corollary 3.2.1, and hence omitted.

For proving Theorem 3.2.2. we observe the following obvious fact.

Lemma 3.2.1 Let $\left\{\xi, \xi_{n}: n \geq 1\right\}$ be a sequence of random variables. If

$$
\lim _{n \rightarrow \infty} P\left\{\xi_{n} \geq \xi\right\}=0
$$

then there is a subsequence $\left\{\xi_{n_{k}}\right\}$ such that

$$
\limsup _{k \rightarrow \infty} \xi_{n_{k}} \leq \xi \quad \text { a.s. }
$$

and so

$$
\liminf _{n \rightarrow \infty} \xi_{n} \leq \xi \quad \text { a.s. }
$$

Proof of Theorem 3.2.2 Firstly, we prove (3.2.9).

If $\lim \sup _{T \rightarrow \infty}\left(\log T / a_{T}\right) / \log \log \log T=\infty$, then there exists a sequence of positive numbers $\left\{T_{n}\right\}$ such that

$$
\lim _{n \rightarrow \infty}\left(\log T_{n} / a_{T_{n}}\right) / \log \log \log T_{n}=\infty
$$

For any $\varepsilon>0, \mu>0$, by Theorem 2.2.2, for $n$ large enough, we have

$$
\begin{aligned}
P\left(\sup _{0 \leq t \leq T_{n}-a_{T_{n}}}\right. & \left.\inf _{f \in S} \sup _{0 \leq x \leq 1}\left|Z_{t . T_{n}}(x)-f(x)\right| \geq \varepsilon\right) \\
\leq & P\left(\cup_{0 \leq t \leq 1-\frac{a_{T_{n}}}{T_{n}}}\left\{\left(2 \frac{a_{T_{n}}}{T_{n}}\left(\log \frac{T_{n}}{a_{T_{n}} \log \log T_{n}}\right)\right)^{-\frac{1}{2}}\left(W\left(t+\frac{a_{T_{n}}}{T_{n}} \cdot\right)-W(t)\right) \in\left(S^{\varepsilon}\right)^{c}\right\}\right) \\
\leq & \frac{T_{n}}{a_{T_{n}}} \exp \left\{-2(1-\mu) \inf _{f \in\left(S^{c}\right)^{c}} I(f) \log \frac{T_{n}}{a_{T_{n}} \log \log T_{n}}\right\} \\
& =\frac{T_{n}}{a_{T_{n}}}\left(\frac{a_{T_{n}} \log \log T_{n}}{T_{n}}\right)^{2(1-\mu) \inf _{f \in\left(S^{\varepsilon}\right)^{c} I(f)} .}
\end{aligned}
$$

Taking $\mu$ small enough, there exists some $\delta>0$ such that

$$
2(1-\mu) \inf _{f \in\left(S^{c}\right)^{c}} I(f)>1+\delta
$$


where $\delta=2(1-\mu) \inf _{f \in\left(S^{e}\right)^{c}} I(f)-1>0$ for small $\mu>0$. Hence

$$
\sum_{k} P\left(\sup _{T_{k} \leq t \leq T_{k+1}-a_{T_{k+1}}} \inf _{f \in S} \sup _{0 \leq x \leq 1}\left|Z_{t, T_{k+1}}(x)-f(x)\right| \leq \varepsilon\right)=\infty,
$$

which. by the Borel-Cantelli lemma, implies that

$$
\liminf _{n \rightarrow \infty} \sup _{T_{k} \leq t \leq T_{k+1}-a_{T_{k+1}}} \inf _{f \in S} \sup _{0 \leq x \leq 1}\left|Z_{t, T_{k+1}}(x)-f(x)\right| \leq \varepsilon \quad \text { a.s. }
$$

Note that

$$
\begin{aligned}
\sup _{0 \leq t \leq T_{k+1}-a_{T_{k+1}}} & \inf _{f \in S} \sup _{0 \leq x \leq 1}\left|Z_{t, T_{k+1}}(x)-f(x)\right| \\
& \leq \sup _{T_{k} \leq t \leq T_{k+1}-a_{T_{k+1}}} \inf _{f \in S} \sup _{0 \leq x \leq 1}\left|Z_{t . T_{k+1}}(x)-f(x)\right| \\
& +2 \sup _{0 \leq t \leq T_{k+1}-T_{k}} \sup _{0 \leq s \leq T_{k}} \gamma_{T_{k+1}}|W(t+s)-W(t)| \\
& :=I_{1}+2 I_{2} .
\end{aligned}
$$

By Theorem 3.1.1 and (3.2.14), we have

$$
\limsup _{k \rightarrow \infty} I_{2} \leq 2\left(\frac{2 T_{k} \log \left(T_{k+1} \log T_{k+1} / T_{k}\right)}{2 a_{T_{k+1}} \log \left(T_{k+1} / T_{k} \log \log T_{k+1}\right)}\right)^{\frac{1}{2}}=0 \quad \text { a.s. }
$$

So, combining (3.2.15) with (3.2.16), we obtain (3.2.9).

Next, we prove (3.2.10). For any $\theta>1$, take $T_{n}=\theta^{n}$. For all $T \geq 3$, there exists integer $n$ such that $T_{n} \leq T \leq T_{n+1}$. Then, noting $T_{n}-a_{T_{n}} \leq T-a_{T}$, for all $f \in S$ we have

$$
\begin{aligned}
\inf _{0 \leq t \leq T-a_{T}} \sup _{0 \leq x \leq 1}\left|Z_{t, T}(x)-f(x)\right| \\
\quad \leq \inf _{0 \leq t \leq T_{n}-a_{T_{n}}} \sup _{0 \leq x \leq 1}\left|Z_{t, T_{n+1}}\left(\frac{a_{T}}{a_{T_{n+1}}} x\right)-f\left(\frac{a_{T}}{a_{T_{n+1}}} x\right)\right| \\
\quad+\left[\left(\frac{\left.\left.a_{T_{n+1}} \log \frac{T_{n+1}}{a_{T_{n}} \log \frac{T_{n+1} \log \log T_{n+1}}{a_{T_{n}} \log \log T_{n}}}\right)^{\frac{1}{2}}-1\right] \sup _{0 \leq t \leq T-a_{T}} \sup _{0 \leq x \leq 1} \gamma_{T}\left|W\left(t+a_{T} x\right)-W(t)\right|}{}+\sup _{f \in S_{a(r)}} \sup _{0 \leq x \leq 1}\left|f\left(\frac{a_{T}}{a_{T_{n+1}}} x\right)-f(x)\right|\right.\right. \\
\quad:=A_{n}+B_{n}+C_{n} .
\end{aligned}
$$


Since. for any $\varepsilon>0$ and positive $\mu$ small enough, there exists some $\delta>0$ silch that

$$
2(1+\mu) \inf _{g \in B(f, \varepsilon)} I(g)<1-\delta
$$

hence, by Theorem 2.2.1 and properties of a Wiener process, for $n$ large enough we have

$$
\begin{aligned}
& P\left(\inf _{0 \leq 1 \leq T_{n}-a_{T_{n}}} \sup _{0 \leq x \leq 1}\left|Z_{t, T_{n+1}}(x)-f(x)\right| \geq \varepsilon\right) \\
& \leq P\left(\min _{0 \leq j \leq\left[\frac{\left.T_{n}-a_{T_{n}}\right]}{a_{T_{n+1}}}\right]} \sup _{0 \leq x \leq 1}\left|Z_{j a_{T_{n+1}}, T_{n+1}}(x)-f(x)\right| \geq €\right) \\
& =\left[P\left(\sup _{0 \leq x \leq 1}\left|Z_{0, T_{n+1}}(x)-f(x)\right| \geq \varepsilon\right)\right]^{\frac{T_{n-a} T_{n}}{a T_{n+1}}-1}
\end{aligned}
$$

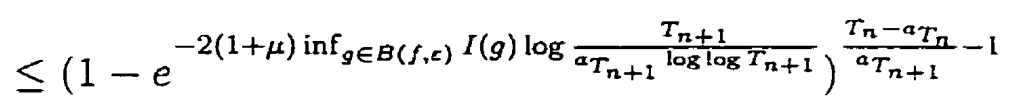

$$
\begin{aligned}
& \leq 9 \exp \left\{-\left(\frac{{ }^{a_{n+1}} \log \log T_{n+1}}{T_{n+1}}\right)^{2(1+\mu) \inf _{g \in B(f, \varepsilon)} I(g)} \frac{T_{n+1}}{a_{T_{n+1}}} \frac{1}{\theta}\right\} \\
& \leq 9 \exp \left\{-\frac{1}{\theta}\left(\frac{T_{n+1}}{a_{T_{n+1}}}\right)^{\delta}\left(\log \log T_{n+1}\right)^{1-\delta}\right\} .
\end{aligned}
$$

Since for any $M>0$, by condition ( $\mathrm{v}$ ), for $n$ large enough

$$
\frac{T_{n+1}}{a_{T_{n+1}}}>M \log \log T_{n+1},
$$

consequently we obtain

$$
P\left(\inf _{0 \leq t \leq T_{n}-a_{T_{n}}} \sup _{0 \leq x \leq 1}\left|Z_{t, T_{n+1}}(x)-f(x)\right| \geq \varepsilon\right) \leq 9 \exp \left\{-2 \log \log T_{n+1}\right\} \leq C(\theta) \frac{1}{n^{2}},
$$

where $C(\theta)$ is a positive constant which only depends on $\theta$. Hence, we have also

$$
\sum_{n} P\left(\inf _{0 \leq t \leq T_{n}-a_{T_{n}}} \sup _{0 \leq x \leq 1}\left|Z_{t, T_{n+1}}(x)-f(x)\right| \geq \varepsilon\right)<\infty .
$$

Therefore, by Borel-Cantelli lemma, we conclude

$$
\limsup _{n \rightarrow \infty} \inf _{0 \leq t \leq T_{n}-a_{T_{n}}} \sup _{0 \leq x \leq 1}\left|Z_{t, T_{n+1}}(x)-f(x)\right|=0 \quad \text { a.s. }
$$


which implies that

$$
\lim _{n \rightarrow \infty} A_{n}=0 \quad \text { a.s. }
$$

Using $T_{n} a_{T_{n}}^{-1} \leq T_{n+1} a_{T_{n+1}}^{-1}$ and Theorem 3.1.3, it is easy to see that

$$
\begin{aligned}
\limsup _{n \rightarrow \infty} B_{n} & \leq 2 \limsup \operatorname{sum}_{n \rightarrow \infty}\left\{\left(\frac{T_{n+1}}{T_{n}} \frac{\log \frac{T_{n+1}}{{ }^{a} T_{n+1} \log \log T_{n+1}}}{\log \frac{T_{n}}{{ }^{a} T_{n+1} \log \log T_{n}}}\right)^{\frac{1}{2}}-1\right\} \\
& \leq 2 \limsup n \rightarrow \infty \\
& \leq 2(\sqrt{\theta}-1) \quad \text { a.s. }
\end{aligned}
$$

Letting $\theta \downarrow 1$, we obtain

$$
\lim _{n \rightarrow \infty} B_{n}=0 \quad \text { a.s. }
$$

By the definition of $S$ and using the same method as in proving (3.2.8), we obtain

$$
\lim _{n \rightarrow \infty} C_{n}=0 \quad \text { a.s. }
$$

Therefore, combining (3.2.18), (3.2.19) and (3.2.20), we obtain (3.2.10). Now, the proof of Theorem 3.2.2 is complete.

\subsection{Functional Limit Theorems for Partial Sums of I.I.D.R.V.}

The fundamental aim of this section is to study functional limit theorems of partial sums with the help of strong approximation methods. Throughout this section we assume that $X_{1}, X_{2}, \cdots$ is a sequence of i.i.d.r.v. with mean zero and variance one, and $\left\{a_{N}\right\}$ is a monotonically non-decreasing sequence of integers satisfying the following conditions:

(i) $0<a_{N} \leq N$, 
(ii) $N / a_{N}$ is monotonically non-decreasing.

Let

$$
\begin{aligned}
& S_{n}=\sum_{k=1}^{n} X_{k}, \\
& S_{n, N}(x)=\beta_{N}\left[\left(S_{n+\left[a_{N} x\right]}-S_{n}\right)+\left(a_{N} x-\left[a_{N} x\right]\right) X_{n+\left[a_{N} x\right]+1}\right], \\
& 0 \leq x \leq 1, \quad 1 \leq n \leq N-a_{N},
\end{aligned}
$$

where $\beta_{N}$ is as in Section 3.1.

Theorem 3.3.1 Let $0<\gamma \leq 1$. Assume that there exists a $t_{0}>0$ such that $E e^{t X_{1}^{\gamma}}$ is finite if $|t|<t_{0}$, and that

$$
\lim _{N \rightarrow \infty} a_{N}(\log N)^{1-\frac{2}{\gamma}}=\infty
$$

Then

$$
\lim _{N \rightarrow \infty} \sup _{1 \leq n \leq N-a_{N}} \inf _{f \in S} \sup _{0 \leq x \leq 1}\left|S_{n . N}(x)-f(x)\right|=0 \quad \text { a.s. }
$$

and for any $f \in S$

$$
\liminf _{N \rightarrow \infty} \sup _{0 \leq x \leq 1}\left|S_{N-a_{N} . N}(x)-f(x)\right|=0 \quad \text { a.s. }
$$

If we also assume that

$$
\text { (iii) } \lim _{N \rightarrow \infty} \frac{\log \frac{N}{a_{N}}}{\log \log N}=r, \quad 0 \leq r \leq \infty
$$

then

$$
\liminf _{N \rightarrow \infty} \sup _{1 \leq n \leq N-a_{N}} \inf _{f \in S_{\alpha(r)}} \sup _{0 \leq x \leq 1}\left|S_{n, N}(x)-f(x)\right|=0 \quad \text { a.s., }
$$

and for any $f \in S_{\alpha(r)}$

$$
\lim _{N \rightarrow \infty} \inf _{1 \leq n \leq N-a_{N}} \sup _{0 \leq x \leq 1}\left|S_{n, N}(x)-f(x)\right|=0 \quad \text { a.s. },
$$

where $\alpha(r)$ is the same as the one in Section 3.2. 
Remark 3.3.1 It is clear that this theorem plavs the same role for partial sums of i.i.d.r.v. with $E e^{t X_{1}^{\gamma}}<\infty$ as Theorem 3.2.1 does for Wiener processes. It is easy to see that Theorem 3.3.1 implies Theorem 3.1.1 of Csörgö and Révész (1981).

Just like getting Corollary 3.2.1, it is also easy to obtain the following result.

Corollary 3.3.1 Under all the conditions of Theorem 3.3.1, we have

$$
\begin{aligned}
& \liminf \inf _{N \rightarrow \infty} \max _{1 \leq n \leq N-a_{N}} \beta_{N}\left|S_{n+a_{N}}-S_{n}\right| \\
&=\lim \inf _{N \rightarrow \infty} \max _{1 \leq n \leq N-a_{N}} \max _{1 \leq k \leq a_{N}} \beta_{N}\left|S_{n+k}-S_{n}\right| \\
&=\alpha(r) \quad \text { a.s. }
\end{aligned}
$$

To prove Theorem 3.3.1, we quote the following strong approximation theorem.

Theorem 3.3.2 (Komlós, Major, Tusnády 1975 and 1976) Let $H(x)>0(x \geq 0)$ be a non-decreasing continuous function such that $x^{-2-\delta} H(x)$ is non-decreasing for some $\delta>0$ and $x^{-1} \log H(x)$ in non-increasing. Assume that $E H\left(\left|X_{1}\right|\right)<\infty$. Then there exists a Wiener process $\{W(t) ; 0 \leq t<\infty\}$ such that

$$
\max _{1 \leq n \leq N}\left|S_{n}-W(n)\right|=O(\operatorname{inv} H(N)) \quad \text { a.s. }
$$

If we also assume that $\lim \inf _{x \rightarrow \infty} \frac{H(\varepsilon x)}{H(x)}>0$ for every $\varepsilon>0$, then we have

$$
\max _{1 \leq n \leq N}\left|S_{n}-W(n)\right|=o(\operatorname{inv} H(N)) \quad \text { a.s }
$$

where inv $H(\cdot)$ is the inverse function of $H(\cdot)$.

Proof of Theorem 3.3.1 Taking $H(x)=e^{t x^{2}}$, combining Theorem 3.2.1 and Theorem 3.3.2, the result follows, since $\beta_{N}(\log N)^{\frac{2}{7}-1} \rightarrow 0$ as $N \rightarrow \infty$.

Theorem 3.3.3 Let $H(x), x>0$, be a non-decreasing continuous function for which the following assumptions hold:

(a) $E H\left(\left|X_{1}\right|\right)<\infty$, 
a Cramér rate function. Define $I^{*}: C_{0}[0,1] \rightarrow \mathbf{R}$ by

$$
I^{*}(f)= \begin{cases}\int_{0}^{1} I\left(f^{\prime}(x)\right) d x, & \text { if } f \text { is an absolutely continuous function } \\ \infty . & \text { otherwise. }\end{cases}
$$

Then, for any $L>0$. the set $K_{L}=\left\{f \in C_{0}[0,1]: I^{*}(f) \leq L\right\}$ is compact. Let

$$
\begin{aligned}
& S_{n}=\sum_{k=1}^{n} X_{k} \\
& S_{n . N}(x . \omega)=a_{N}^{-1}\left[\left(S_{n+\left[a_{N} x\right]}(\omega)-S_{n}(\omega)\right)+\left(a_{N} x-\left[a_{N} x\right]\right) X_{n+\left[a_{*} \times[+1\right.}(\omega)\right], \\
& 0 \leq x \leq 1, \quad 1 \leq n \leq N-a_{N} .
\end{aligned}
$$

where $a_{N}=[c \log N]$. and $c$ is a positive constant.

Erdős and Rényi (1970) proved the following important result.

Theorem 3.4.1 We have

$$
\lim _{N \rightarrow \infty} \max _{0 \leq n \leq N-a_{N}} \frac{S_{n+a_{N}}-S_{n}}{a_{N}}=\sup \left\{u>0: I(u) \leq \frac{1}{c}\right\} \quad \text { a.s. }
$$

Let $L_{c}=\left\{f \in C_{0}^{\prime}[0.1]: I^{*}(f) \leq \frac{1}{c}\right\}$. Sanchis (1994) presented and proved the following functional limit theorem for the Erdős and Rényi law of large numbers.

Theorem 3.4.2 We have

$$
\lim _{N \rightarrow \infty} \max _{0 \leq n \leq N-a_{N}} \inf _{f \in L_{c}} \sup _{0 \leq x \leq 1}\left|S_{n . N}(x)-f(x)\right|=0 \quad \text { a.s. }
$$

and, for any $f \in L_{c}$, we have

$$
\liminf _{N \rightarrow \infty} \min _{0 \leq n \leq N-a_{N}} \sup _{0 \leq x \leq 1}\left|S_{n . N}(x)-f(x)\right|=0 \quad \text { a.s. }
$$

Remark 3.4.1 From Theorem 3.4.2, it is easy to obtain that

$$
\limsup _{N \rightarrow \infty} \max _{0 \leq n \leq N-a_{N}} \frac{S_{n+a_{N}}-S_{n}}{a_{N}}=\sup \left\{u>0: I(u) \leq \frac{1}{c}\right\} \quad \text { a.s. }
$$


Theorem 3.4.2 does not however imply the Erdös and Renyi law (cf. Theorem 3.4.1). for it is a "lim" result. Hence it is natural to ask the question: what is the functional limit theorem that one needs to deduce also the Erdös and Renyi law? The answer to this question is the following theorem.

Theorem 3.4.3 We have (3.4.2), and, for any $f \in L_{c}$, we have

$$
\lim _{N \rightarrow \infty} \min _{0 \leq n \leq N-a_{N}} \sup _{0 \leq x \leq 1}\left|S_{n . N}(x)-f(x)\right|=0 \quad \text { a.s. }
$$

Corollary 3.4.1 (3.4.2) and (3.4.5) imply (3.4.1).

The proof of Theorem 3.4.3 relies heavily on the following large deviations result.

Lemma 3.4.1 (Borovkov, 1967) Define the stochastic processes $V_{n}$ by

$$
V_{n}(t, \omega)=\frac{S_{[n t]}(\omega)}{n}+\frac{n t-[n t]}{n} X_{[n t]+1}(\omega), \quad 0 \leq t \leq 1, \omega \in \Omega .
$$

Define the sequence of probability measures $\left\{Q_{n}\right\}$ on $C_{0}[0,1]$ by $Q_{n}(\Gamma)=P\left(V_{n} \in \Gamma\right)$. Then $\left\{Q_{n}\right\}$ satisfies the full large deviation principle with rate function $I^{*}$.

Proof of Theorem 3.4.1 By Theorem 3.4.2, it suffices to show that (3.4.5). For any $\varepsilon>0$ and $\mu>0$, by Lemma 3.4.1, for $n$ large enough we have

$$
\begin{aligned}
& P\left(\min _{0 \leq n \leq N-a_{N}} \sup _{0 \leq x \leq 1}\left|S_{n, N}(x)-f(x)\right| \geq \varepsilon\right) \\
& \quad \leq P\left(\min _{0 \leq i \leq\left[N / a_{N}\right]-1} \sup _{0 \leq x \leq 1}\left|S_{i a_{N}, N}(x)-f(x)\right| \geq \varepsilon\right) \\
& \quad \leq\left\{1-P\left(\sup _{0 \leq x \leq 1}\left|S_{i a_{N}, N}(x)-f(x)\right|<\varepsilon\right)\right\}^{\frac{N}{a_{N}}-2} \\
& \quad \leq\left\{1-e^{-(1+\mu) \inf _{g \in B(f, \varepsilon)} I^{*}(g) a_{N}}\right\}^{\frac{N}{a_{N}}-2} \\
& \quad \leq\left\{1-e^{-\frac{1-\delta}{c} \cdot \log N}\right\}^{\frac{N}{a_{N}}-2} \\
& \leq 9 e^{-\frac{N^{\delta}}{c \log N}}
\end{aligned}
$$




\section{Chapter 4}

\section{Subsequences of Increments for a Wiener Process}

In this chapter, we discuss functional limit theorems for subsequences of increments of a Wiener process. The motivation comes from Gut (1986) (cf. Section 4.1) and Vasudeva and Savitha (1993) (cf. Section 4.2). We may view this chapter as a "sequential version" of the previous chapter.

\subsection{The Laws of the Iterated Logarithm for Sub- sequences}

Let $\left\{X_{n}\right\}$ be a sequence of independent random variables having a common distribution with $E X_{n}=0$ and $\operatorname{Var}\left(X_{n}\right)=1$. The well known law of the iterated logarithm is

$$
\limsup _{n \rightarrow \infty} \frac{S_{n}}{\sqrt{2 n \log \log n}}=1 \quad \text { a.s. }
$$

Moreover, with probability one, the set of limit points of $\left\{\frac{S_{n}}{\sqrt{2 n \log \log n}}: n \geq 3\right\}$ is the interval $[-1,1]$. Concerning (4.1.1) Gut (1986) posed the following two questions.

Question 1 What is the class of subsequences for which the law of the iterated logarithm still holds true? 
Question 2 For any $r \leq 1$, what is the class of subsequences for which the "limsup" is $r$ instead of 1 in the law of the iterated logarithm?

The answers Gut (1986) gave to these two questions are as follows.

Theorem 4.1.1 Let $\left\{n_{k}\right\}$ be some subsequence of integers such that

$$
\limsup _{k \rightarrow \infty} \frac{n_{k+1}}{n_{k}}<\infty
$$

Then we have

$$
\limsup _{n \rightarrow \infty} \frac{S_{n_{k}}}{\sqrt{2 n_{k} \log \log n_{k}}}=1 \quad \text { a.s. }
$$

Theorem 4.1.2 Let $\left\{n_{k}\right\}$ be some subsequence of integers such that

$$
\lim _{k \rightarrow \infty} \frac{n_{k+1}}{n_{k}}=\infty
$$

Then we have.

$$
\limsup _{n \rightarrow \infty} \frac{S_{n_{k}}}{\sqrt{2 n_{k} \log \log n_{k}}}=r \quad \text { a.s. }
$$

where

$$
r:=\inf \left\{d>0: \sum_{k}\left(\frac{1}{\log n_{k}}\right)^{d^{2}}<\infty\right\} .
$$

Remark 4.1.1 These two theorems can also be considered as respective corollaries of Theorems 4.3.1 and 4.3.2 of this chapter (cf. Remarks 4.2.1 and 4.2.3, respectively).

\subsection{How Large Are Subsequences of Increments for a Wiener Process}

Let $W:=\{W(t), 0 \leq t<\infty\}$ be a standard Wiener process. Let $a_{t}, t>0$, be a nondecreasing function of $t$ with $0<a_{t} \leq t$ and $t^{-1} a_{t}$ nonincreasing. Following the Csörgö and Révész book (1981), and motivated by Gut (1986), Vasudeva and 
Savitha (1993) proved similar results for increments of a Wiener process. However. their results and proofs are only suitable to deal with sequential versions of the type of increments $W\left(t+a_{t}\right)-W(t)$. Consequently, we cannot obtain Gut's results from those of Vasudeva and Savitha. Here, we present generalizations of Gut's results for lag increments of a Wiener process (cf. Step 1 on page 735 in the proof of Theorem 1 in Csörgö and Révész (1979), and Theorem 3.1 of Hanson and Russo (1983)). Let

$$
\begin{gathered}
Y(t)=\beta_{t_{k}}\left(W(t)-W\left(t-a_{t}\right)\right) \\
\beta_{t_{k}}^{-1}=\left(2 a_{t_{k}}\left(\log \left(t_{k} / a_{t_{k}}\right)+\log \log t_{k}\right)\right)^{1 / 2}
\end{gathered}
$$

Theorem 4.2.1 Let $a_{t}, t>0$, be a nondecreasing function of $t$ with $0<a_{t} \leq t$ and $t^{-1} a_{t}$ nonincreasing. Let $\left\{t_{k}\right\}$ be any increasing sequence diverging to $\infty$ such that

$$
\limsup _{k \rightarrow \infty} \frac{t_{k+1}-t_{k}}{a_{t_{k}}}<\infty
$$

Then

$$
\limsup _{k \rightarrow \infty} Y\left(t_{k}\right)=\limsup _{k \rightarrow \infty} \sup _{0 \leq s \leq a_{t_{k}}} \beta_{t_{k}}\left(W\left(t_{k}-a_{t_{k}}+s\right)-W\left(t_{k}-a_{t_{k}}\right)\right)=1 \quad \text { a.s. }
$$

Remark 4.2.1 It is easy to extend this theorem to partial sums of i.i.d. random variables under appropriate conditions by strong approximation theorems (cf. Csörgö and Révész 1981). In particular, we can obtain Theorem 4.1.1 via this way by taking $a_{t}=t$

Remark 4.2.2 We do not prove this theorem directly because it follows from our more general Theorem 4.3.1.

Theorem 4.2.2 Let $a_{t}, t>0$, be a nondecreasing function of $t$ with $0<a_{t} \leq t$ and $t^{-1} a_{t}$ nonincreasing and put $g(t)=(t \log t) / a_{t}$. Let $\left\{t_{k}\right\}$ be any increasing sequence 


\subsection{Functional Strong Limit Theorems}

The aim of this section is to present and prove the functional limit theorems for increments of a Wiener process, which imply all the results in Sections 4.1 and 4.2.

Theorem 4.3.1 Let $a_{t}, t>0$, be a nondecreasing function of $t$ with $0<a_{t} \leq t$ and $t^{-\mathrm{I}} a_{t}$ nonincreasing. Let $\left\{t_{k}\right\}$ be any increasing sequence diverging to $\infty$ such that

$$
\limsup _{k \rightarrow \infty} \frac{t_{k+1}-t_{k}}{a_{t_{k}}}<\infty
$$

Then the sequence of functions

$$
\left\{\xi_{t_{k}}(\cdot)\right\}:=\left\{\beta_{t_{k}}\left(W\left(t_{k}-a_{t_{k}}+x a_{t_{k}}\right)-W\left(t_{k}-a_{t_{k}}\right)\right), 0 \leq x \leq 1\right\}_{k=1}^{\infty}
$$

is relatively compact in $C_{0}[0,1]$, with probability one, and the set of its limit points is $S$, where $S$ is defined by

$$
S=\left\{f(\cdot) \in C_{0}[0,1]: I(f) \leq \frac{1}{2}\right\}
$$

as in Section 2.3.

Remark 4.3.1 We can obtain Theorem 4.2.1 from this theorem by taking $x=1$ and using $\sup _{f \in S} f(1)=1$.

Theorem 4.3.2 Let $a_{t}, t>0$, be a nondecreasing function of $t$ with $0<a_{t} \leq t$ and $t^{-1} a_{t}$ nonincreasing. Let $\left\{t_{k}\right\}$ be any increasing sequence diverging to $\infty$ such that

$$
\lim _{k \rightarrow \infty} \frac{t_{k+1}-t_{k}}{a_{t_{k}}}=\infty
$$

Then the sequence of functions

$$
\left\{\xi_{t_{k}}(\cdot)\right\}:=\left\{\beta_{t_{k}}\left(W\left(t_{k}-a_{t_{k}}+x a_{t_{k}}\right)-W\left(t_{k}-a_{t_{k}}\right)\right), 0 \leq x \leq 1\right\}_{k=1}^{\infty}
$$


is relatively compact in $C_{0}[0,1]$, with probability one, and the set of its limit points is

$$
S_{r}:=\left\{f \in C_{0}[0,1]: I(f) \leq r^{2} / 2\right\}
$$

where

$$
r:=\inf \left\{d>0: \sum_{k}\left(g\left(t_{k}\right)\right)^{-d^{2}}<\infty\right\} .
$$

Remark 4.3.2 We can obtain Theorem 4.2.2 from this theorem by taking $x=1$ and using $\sup _{f \in S_{r}} f(\mathbb{1})=r$.

Remark 4.3.3 By Theorems 4.3.1 and 4.3.2. the set of limit points $S_{r}$ depends both on $\left\{t_{k}\right\}$ and the function $a_{t}, t>0$. We present the following examples.

(i) Take $t_{k}=k^{\alpha}(\alpha \geq 1)$, and $a_{t}=t^{q}(0 \leq q \leq 1)$. Then, we have

$$
r= \begin{cases}1, & (\alpha-1) / \alpha \leq q \leq 1, \\ 1 / \sqrt{(1-q) \alpha}, & 0 \leq q<(\alpha-1) / \alpha .\end{cases}
$$

(ii) Take $t_{k}=\alpha^{k}(\alpha>1)$, and $a_{t}=t^{q}(0 \leq q \leq 1)$. Then, we have

$$
r= \begin{cases}1, & q=1 . \\ 0, & 0 \leq q<1 .\end{cases}
$$

Remark 4.3.4 It is easy to extend Theorems 4.3 .1 and 4.3.2 to partial sums of i.i.d. random variables under appropriate conditions by strong approximation theorems (cf. Csörgö and Révész 1981).

Proof of Theorem 4.3.1 First of all, by Theorem 3.1.2, we have

$$
\lim _{k \rightarrow \infty} \inf _{f \in S}\left\|\xi_{t_{k}}-f\right\|=0 \quad \text { a.s. }
$$

Hence, it suffices to show that for all $f \in S$

$$
\liminf _{k \rightarrow \infty}\left\|\xi_{t_{k}}-f\right\|=0 \quad \text { a.s. }
$$


Under the given conditions, we can let $\lim _{t \rightarrow \infty}\left(a_{t} / t\right)=\rho(0 \leq \rho \leq 1)$. Throughout this proof. we assume that

$$
\theta>\limsup _{k \rightarrow \infty} \frac{t_{k+1}-t_{k}}{a_{t_{k}}}+1
$$

In case of $0 \leq \rho<1$, define the sequence $\left\{u_{k}\right\}$ by $u_{1}=1$ and for $k \geq 1$

$$
u_{k+1}-\theta a_{u_{k}}=u_{k}
$$

For any $\delta>0$. let $B(f, \delta):=\{h:\|f-h\|<\delta\}$. By Theorem 2.2.1. for any $\mu>0$, we have

$$
\begin{aligned}
P\left(\xi_{t} \in B(f, \delta)\right) & \geq \exp \left\{-2(1+\mu) \inf _{h \in B(f, \delta)} I(h) \log g(t)\right\} \\
& =g(t)^{-2(1+\mu) \inf _{h \in B(f . \delta)} I(h)},
\end{aligned}
$$

provided that $t$ is large enough. Since $\inf _{h \in B(f, \delta)} I(h)<\frac{1}{2}$, hence we can take $\mu$ small enough such that

$$
\eta:=2(1+\mu) \inf _{h \in B(f, \delta)} I(h)<1
$$

Thus, there exists a positive integer $K_{1}$ such that

$$
\sum_{k=K_{1}}^{\infty} P\left(\xi_{u_{k}} \in B(f, \delta)\right) \geq \sum_{k=K_{1}}^{\infty}\left(\frac{a_{u_{k}}}{u_{k} \log u_{k}}\right)^{\eta}
$$

Since $-\log (1-x) \leq K_{\varepsilon} x$ for all $x \in(0,1-\varepsilon)$ and some $K_{\varepsilon}>0$, we have

$$
\log u_{n}=\sum_{k=K_{1}}^{n} \log \frac{u_{k}}{u_{k-1}}=-\sum_{k=K_{1}}^{n} \log \left(1-\frac{\theta a_{u_{k-1}}}{u_{k-1}}\right) \leq K_{\varepsilon} \theta \sum_{k=K_{1}}^{n} \frac{a_{u_{k-1}}}{u_{k-1}} .
$$

Consequently

$$
\sum_{k=K_{1}}^{n}\left(\frac{a_{u_{k}}}{u_{k} \log u_{k}}\right)^{\eta} \geq C(\theta, \varepsilon)\left(\sum_{k=K_{1}}^{n} \frac{a_{u_{k}}}{u_{k}}\right)^{1-\eta}
$$


where $C(\theta, \varepsilon)$ is a positive constant that only depends on $\theta$ and $\varepsilon$. Thus $\sum_{k=1}^{\infty} \frac{a_{u_{k}}}{u_{u_{k}}}=\infty$, which, in turn. implies that

$$
\sum_{k=K_{1}}^{\infty} P\left(\xi_{u_{k}} \in B(f, \delta)\right) \geq \sum_{k=K_{1}}^{\infty}\left(\frac{a_{u_{k}}}{u_{k} \log u_{k}}\right)^{\eta}=\infty .
$$

By the monotonicity of the function $g$ as defined in Theorem 4.2 .2 and the above result, we obtain

$$
\sum_{k=K_{1}}^{\infty} P\left(\xi_{u_{2 k}} \in B(f, \delta)\right) \geq \sum_{k=K_{1}}^{\infty}\left(\frac{a_{u_{2 k}}}{u_{2 k} \log u_{2 k}}\right)^{\eta}=\infty
$$

Let $\left\{t_{k}^{\prime}\right\}$ be a subsequence of $\left\{t_{k}\right\}$ such that $u_{k} \leq t_{k}^{\prime}<u_{k+1}$ for all $k$ large enough. It is easy to show that such a subsequence exists by the construction of $\left\{u_{k}\right\}$. Considering the odd subsequence $\left\{t_{2 k-1}^{\prime}\right\}$ of $\left\{t_{k}^{\prime}\right\}$, by (4.3.5) we have

$$
\begin{aligned}
\sum_{k=K_{1}}^{\infty} P\left(\xi_{t_{2 k-1}^{\prime}} \in B(f, \delta)\right) & \geq \sum_{k=K_{1}}^{\infty}\left(\frac{a_{t_{2 k-1}^{\prime}}}{t_{2 k-1}^{\prime} \log t_{2 k-1}^{\prime}}\right)^{\eta} \\
& \geq \sum_{k=K_{1}}^{\infty}\left(\frac{a_{u_{2 k}}}{u_{2 k} \log u_{2 k}}\right)^{\eta}=\infty
\end{aligned}
$$

Noting that $\left\{\xi_{t_{2 k-1}^{\prime}}(\cdot)\right\}$ is an independent random variable subsequence. via the BorelCantelli lemma. we obtain

$$
\liminf _{k \rightarrow \infty}\left\|\xi_{t_{2 k-1}^{\prime}}-f\right\| \leq \delta \quad \text { a.s. }
$$

Letting $\delta \downarrow 0$, we get (4.3.4) from (4.3.6).

In case of $\rho=1$, define $u_{1}=1$, and for $k \geq 2, u_{k}=\theta^{k}$. Take a subsequence of $\left\{t_{k}\right\}$, say $\left\{\hat{t}_{k}\right\}$, such that $u_{k} \leq \hat{t}_{k} \leq u_{k+1}$ for all $k$ large enough, and let $\alpha_{t}=(2 t \log \log t)^{-\frac{1}{2}}$. 
Then we have

$$
\begin{aligned}
|| \xi_{\hat{t}_{2 k+1}}-f \| \leq & \operatorname{sip}_{0 \leq x \leq 1}\left|\xi_{i_{2 k+1}}-\alpha_{\hat{t}_{2 k+1}} W\left(x \hat{t}_{2 k+1}\right)\right| \\
& +\sup _{0 \leq x \leq 1}\left|\alpha_{\hat{t}_{2 k+1}} W\left(x \hat{t}_{2 k+1}\right)-f(x)\right| \\
:= & A_{1}(k)+A_{2}(k) .
\end{aligned}
$$

Firstly, we prove that

$$
\limsup _{k \rightarrow \infty} A_{1}(k)=0 \quad \text { a.s. }
$$

In fact, we have

$$
\begin{aligned}
A_{1}(k) \leq & \left|1-\frac{\alpha_{i_{2 k+1}}}{\hat{\beta}_{i_{2 k+1}}}\right| \beta_{\hat{t}_{2 k+1}} \sup _{0 \leq x \leq 1}\left|W\left(\hat{t}_{2 k+1}-a \hat{t}_{2 k+1}+x a \hat{t}_{2 k+1}\right)-W\left(\hat{t}_{2 k+1}-a \hat{t}_{2 k+1}\right)\right| \\
& +2 \alpha_{t_{2 k+1}} \sup _{0 \leq x \leq 1}\left|W\left(\hat{t}_{2 k+1}-a \hat{t}_{2 k+1}+x a \hat{t}_{2 k+1}\right)-W\left(x \hat{t}_{2 k+1}\right)\right| \\
& :=A_{11}(k)+A_{12}(k) .
\end{aligned}
$$

By (4.3.3), with probabilty one, we have

$$
\limsup _{k \rightarrow \infty} A_{11}(k) \leq 2 \limsup _{k \rightarrow \infty}\left|1-\frac{\alpha_{t_{2 k+1}}}{\beta_{\hat{t}_{2 k+1}}}\right|=0
$$

Noting that

$$
A_{12}(k) \leq 2 \alpha_{t_{2 k+1}} \sup _{|t-s|<\hat{t}_{2 k+1}-a \hat{t}_{2 k+1}}|W(t)-W(s)|,
$$

via well known results for increments of Wiener processes due to Csörgö and Révész $(1979,1981)$ (cf. Theorem 3.1.1 in Chapter 3), we conclude

$$
\limsup _{k \rightarrow \infty} A_{12}(k)=0 \quad \text { a.s. . }
$$

Therefore, combining (4.3.8) with (4.3.9), we obtain (4.3.7).

Next, we prove that

$$
\liminf _{k \rightarrow \infty} A_{2}(k)=0 \quad \text { a.s. }
$$


Let

$$
\eta_{\theta, \hat{t}_{2 k+1}}(x)= \begin{cases}0 . & 0 \leq x \leq \frac{1}{\theta} \\ \alpha_{\bar{t}_{2 k+1}}\left(W\left(x \bar{t}_{2 k+1}\right)-W\left(\hat{t}_{2 k+1} / \theta\right)\right), & \frac{1}{\theta} \leq x \leq 1\end{cases}
$$

and

$$
f_{\theta}(x)= \begin{cases}0, & 0 \leq x<\frac{1}{\theta}, \\ f(x)-\frac{1}{\theta}, & \frac{1}{\theta} \leq x \leq 1 .\end{cases}
$$

Then, using (4.3.6) and continuity of $f$. it suffices to show that, for any $\delta>0$,

$$
\liminf _{k \rightarrow \infty}\left\|\eta_{i_{2 k+1}}-f_{\theta}\right\| \leq \delta \quad \text { a.s. }
$$

For any $\mu>0$, by Theorem 2.2.1, for $k$ large enough we have

$$
P\left(\left\|\eta_{\hat{t}_{2 k+1}}-f_{\theta}\right\|<\delta\right) \geq \exp \left\{-2(1+\mu) \inf _{h \in B\left(f_{\theta}, \delta\right)} \int_{0}^{1-\frac{1}{\theta}}\left(\frac{\partial h(s)}{\partial s}\right)^{2} d s \log g\left(\hat{t}_{2 k+1}\right)\right\} .
$$

Since

$$
\int_{0}^{1-\frac{1}{\theta}}\left(\frac{\partial f_{\theta}(s)}{\partial s}\right)^{2} d s \leq \int_{0}^{1-\frac{1}{\theta}}\left(\frac{\partial f(s)}{\partial s}\right)^{2} d s \leq 1,
$$

hence, we can take $\mu$ small enough such that

$$
\eta:=2(1+\mu) \inf _{h \in B\left(f_{\theta}, \delta\right)} \int_{0}^{1-\frac{1}{\theta}}\left(\frac{\partial h(s)}{\partial s}\right)^{2} d s<1 .
$$

Therefore,

$$
\begin{aligned}
\sum_{k} P\left(\left\|\eta_{\hat{t}_{2 k+1}}-f_{\theta}\right\|<\delta\right) & \geq \sum_{k}\left(\log \hat{t}_{2 k+1}\right)^{-\eta} \\
& \geq \sum_{k}\left(\log u_{2 k+2}\right)^{-\eta} \\
& \geq C \sum_{k} k^{-\eta}=\infty,
\end{aligned}
$$

where $C$ is some positive constant. Since $\left\{\eta_{\bar{t}_{2 k+1}}\right\}$ is a sequence of independent random variables, via the Borel-Cantelli lemma, we get (4.3.11). Combining (4.3.7) and (4.3.11), we obtain (4.3.4). Now, the proof of Theorem 4.3.1 is complete.

Proof of Theorem 4.3.2 We will establish that

$$
\lim _{k \rightarrow \infty} \inf _{f \in S_{r}}\left\|\xi_{t_{k}}-f\right\|=0 \quad \text { a.s. }
$$


Thus $\left\{\xi_{t_{k}}\right\}_{k=K^{-}}^{\infty}$ is a sequence of independent random variables.

If $\rho=0$, let

$$
\begin{aligned}
& D_{K}:=\left\{k \geq K: \frac{t_{k+1}}{t_{k}} \leq 2\right\}, \\
& E_{K}:=\left\{k \geq K: \frac{t_{k+1}}{t_{k}}>2\right\} .
\end{aligned}
$$

Assume $k \in D_{K}$, and using $\lim _{k \rightarrow \infty} \frac{t_{k+1}-t_{k}}{a_{t_{k}}}=\infty$, for $K$ large enough, we have

$$
t_{k+1}-t_{k} \geq 3 a_{t_{k}}>a_{t_{k+1}}
$$

Assume $k \in E_{K}$, and using $\lim _{k \rightarrow \infty} \frac{t_{k+1}}{a_{t_{k+1}}}=\infty$, for $K$ large enough, we have also

$$
\begin{aligned}
\frac{t_{k+1}-t_{k}}{a_{t_{k+1}}} & =\frac{t_{k+1}}{a_{t_{k+1}}}\left(1-\frac{t_{k}}{t_{k+1}}\right) \\
& \geq \frac{1}{2} \frac{t_{k+1}}{a_{t_{k+1}}} \\
& >1 .
\end{aligned}
$$

Therefore, for $K$ large enough, with $k \geq K$ we always have

$$
t_{k+1}-a_{t_{k+1}}>t_{k}
$$

which implies that $\left\{\xi_{t_{k}}\right\}_{k=K}^{\infty}$ is a sequence of independent random variables.

For any $f \in S_{r}$ and $\delta>0$, we have

$$
\inf _{\|h-f\|<\delta} I(h)<r^{2} / 2
$$

Hence, taking positive $\mu$ small enough, we have

$$
2(1+\mu) \inf _{\|h-f\|<\delta} I(h)<r^{2}
$$




\section{Chapter 5}

\section{Two-Parameter Wiener Processes}

\subsection{Introduction}

The original motivation for this work comes from a result of Gantert (1993) which describes a version of Strassen's law of the iterated logarithm for one-parameter Wiener process for small time. Let $\{W(t), 0 \leq t<\infty\}$ be one-parameter standard Wiener process, and let $C_{0}[0,1]$ be the space of continuous functions on the closed interval $[0,1]$ which vanish at the origin. Gantert (1993) showed that with probability one

$$
\psi_{n}(x)=\frac{W\left(\frac{1}{n} x\right)}{\sqrt{\frac{2}{n} \log \log n}}, \quad 0 \leq x \leq 1, \quad n=1,2, \cdots
$$

is relatively compact in $C_{0}[0,1]$, and the set of its limit points (as $n \rightarrow \infty$ ) is $K_{1}$, where $K_{1} \subset C_{0}[0,1]$ is the set of absolutely continuous functions $f$ for which

$$
\int_{0}^{1}\left(f^{\prime}(x)\right)^{2} d x \leq 1
$$

In this chapter, we are interested in Strassen-type laws for two-parameter Wiener processes, including that for small time. Let $W:=\left\{W(x, y):(x, y) \in[0, \infty)^{2}\right\}$ be a two-parameter Wiener process(for existence and properties cf., for example, CsörgöRévész 1981), and let $C_{0}[0,1]^{2}$ be the space of functions that are continuous and vanishing along the axes on $[0,1]^{2}$. Zimmermann (1972), Paranjape and Park (1973), 
and Pruitt and Orey (1973) studied various laws of the iterated logarithm for $\mathrm{W}^{\circ}$. For Strassen-type laws of the iterated logarithm that are related to the results of this chapter, we refer to Wichura (1973) and Park (1974) (cf. also Section 5.3 of this thesis). In Section 5.2, we introduce a large deviation result for $W$, which is a basic tool in proving our results. In Section 5.3, we prove several Strassen-type laws of the iterated logarithm for $W$ in $C_{0}[0.1]^{2}$. which imply most of the formerly known related laws of the iterated logarithm for two-parameter Wiener processes and Strassen's law as well in the one-parameter case. In Section 5.4, we consider some extensions of results in Section 5.3 for the Kiefer and the empirical processes.

\subsection{A Large Deviation for the Two-Parameter Wiener Process}

For any $\varepsilon>0$, let $P_{\varepsilon}$ be probability measure on $C_{0}[0,1]^{2}$ corrosponding to $\sqrt{\varepsilon} W$. Let

$$
I(f)= \begin{cases}\frac{1}{2} \int_{0}^{1} \int_{0}^{1}\left(\frac{\partial^{2} f}{\partial y \partial x}\right)^{2} d x d y, & \begin{array}{l}
\text { if } f \text { is an absolutely continuous function } \\
\text { with a square integrable } \frac{\partial^{2} f}{\partial y \partial x}
\end{array} \\
\infty, & \text { otherwise. }\end{cases}
$$

Then, it is easy to show that $I(f)$ is lower semi-continuous and $\{f: I(f) \leq a\}$ is compact for any fixed $a>0$. We prove the following large deviation result.

Theorem 5.2.1 For any closed set $C \subset C_{0}[0,1]^{2}$, we have

$$
\limsup _{\varepsilon \rightarrow 0} \varepsilon \log P_{\S}(C) \leq-i n f_{f \in C} I(f),
$$

and for any open set $G \subset C_{0}[0,1]^{2}$, we have

$$
\liminf _{\varepsilon \rightarrow 0} \varepsilon \log P_{\varepsilon}(G) \geq-i n f_{f \in G} I(f) .
$$


Remark 5.2.1 If we replace $C_{0}[0,1]^{2}$ by $C_{0}[0, a]^{2}(a>0)$, then the result corresponding to Theorem 5.2.1 is also true.

For proving the theorem, we have the following lemma.

Lemma 5.2.1 (cf. Lemma 1.11.1, Csörgö-Révész 1981). For any $\mu>0$ there exists a constant $C=C(\mu)>0$ such that the inequality

$$
P\left(\sup _{0 \leq s \leq h} \sup _{(x, y) \in[0,1]^{2}}|W(x, y+s)-W(x, y)| \geq v \sqrt{h}\right) \leq C h^{-1} \exp \left\{-\frac{v^{2}}{2+\mu}\right\}
$$

holds for every positive $v$ and $0<h<1$.

Proof of Theorem 5.2.1 Firstly we want to verify (5.2.1). For any positive integer $n$ and any $f \in C_{0}[0,1]^{2}$ define $f_{n} \in C_{0}[0,1]^{2}$ by

$f_{n}(x, y)=a_{n}(k, i) x y+b_{n}(k, i) x+c_{n}(k, i) y+d_{n}(k, i), \quad(x, y) \in I_{n}(k, i), 0 \leq k, i \leq n$ where

$$
\begin{gathered}
a_{n}(k, i)=n^{2}\left[f\left(\frac{k+1}{n}, \frac{i+1}{n}\right)-f\left(\frac{k+1}{n}, \frac{i}{n}\right)-f\left(\frac{k}{n}, \frac{i+1}{n}\right)+f\left(\frac{k}{n}, \frac{i}{n}\right)\right], \\
I_{n}(k, i)=\left\{(x, y):(x, y) \in\left[\frac{k}{n}, \frac{k+1}{n}\right] \times\left[\frac{i}{n}, \frac{i+1}{n}\right]\right\},
\end{gathered}
$$

and $b_{n}(k, i), c_{n}(k, i), d_{n}(k, i)$ are constants such that

$$
f(x, y)=f_{n}(x, y), \quad(x, y) \in\left\{\left(\frac{k}{n}, \frac{i}{n}\right)\left(\frac{k+1}{n}, \frac{i}{n}\right),\left(\frac{k}{n}, \frac{i+1}{n}\right),\left(\frac{k+1}{n}, \frac{i+1}{n}\right)\right\}
$$




$$
k=0,1,2,3, \cdots, i=0.1,2.3, \cdots
$$

For any $\delta>0$, let

$$
C^{\delta}=\left\{g \in C_{0}[0,1]^{2}: \inf _{f \in C}\|f-g\|<\delta\right\}
$$

Then

$$
\begin{aligned}
P_{\bar{\varepsilon}}(C) & =P_{\varepsilon}\left(C \cap\left(\left\|f_{n}-f\right\|<\delta\right)\right)+P_{\S}\left(C^{\prime} \cap\left(\left\|f_{n}-f\right\| \geq \delta\right)\right) \\
& \leq P_{\varepsilon}\left(f_{n} \in C^{\delta}\right)+P_{\varepsilon}\left(\left\|f_{n}-f\right\| \geq \delta\right) \\
& :=A+B .
\end{aligned}
$$

Let $l_{\delta}=i n f_{f \in C^{\delta}} I(f)$. Then

$$
\begin{aligned}
A & \leq P_{\varepsilon}\left(I\left(f_{n}\right) \geq l_{\delta}\right) \\
& =P_{\varepsilon}\left(\frac{1}{2 n^{2}} \sum_{k=1}^{n} \sum_{i=1}^{n} a_{n}^{2}(k, i) \geq l_{\delta}\right) .
\end{aligned}
$$

Since $\frac{1}{\xi \pi^{2}} \sum_{k=1}^{n} \sum_{i=1}^{n} a_{n}^{2}(k, i)$ is a $\chi_{n^{2}}^{2}$ random variable under $P_{\xi}$, we have

$$
A \leq \frac{1}{\Gamma\left(\frac{n^{2}}{2}\right) 2^{\frac{n^{2}}{2}}} \int_{\frac{2 l_{\delta}}{\epsilon}}^{\infty} \exp \left\{-\frac{x}{2}\right\} x^{\frac{n^{2}}{2}-1} d x \leq C(n) \exp \left\{-\frac{1-\mu}{\varepsilon} l_{\delta}\right\},
$$

where $C(n)$ is a constant that depends on $n$, and $\mu$ is any positive number. Therefore

$$
\limsup _{\varepsilon \rightarrow 0} \varepsilon \log A \leq-(1-\mu) l_{\delta}
$$

and letting $\mu \rightarrow 0$, we obtain

$$
\underset{\varepsilon \rightarrow 0}{\limsup } \varepsilon \log A \leq-l_{\delta}
$$


On the other hand, by Lemma 5.2 .1 we have

$$
\begin{aligned}
& B=P_{\varepsilon}\left(\left\|f_{n}-f\right\| \geq \delta\right) \\
& =P\left(\sqrt{\varepsilon} \sup _{(x, y) \in[0,1]^{2}}\left|W(x, y)-W_{n}(x, y)\right| \geq \delta\right) \\
& =P\left(\max _{1 \leq k, i \leq n} \sup _{(x, y) \in I_{n}(k, i)}\left|W(x, y)-W_{n}(x, y)\right| \geq \frac{\delta}{\sqrt{\varepsilon}}\right) \\
& \leq \sum_{1 \leq k, i \leq n} P\left(\sup _{(x, y) \in I_{n}(k, i)}\left|W(x, y)-W\left(\frac{k}{n}, \frac{i}{n}\right)\right| \geq \frac{\delta}{2 \sqrt{\varepsilon}}\right) \\
& \leq \sum_{1 \leq k, i \leq n}\left[P\left(\sup _{(x, y) \in I_{n}(k, i)}\left|W(x, y)-W\left(\frac{k}{n}, y\right)\right| \geq \frac{\delta}{4 \sqrt{\varepsilon}}\right)\right. \\
& \left.+P\left(\sup _{(x, y) \in I_{n}(k, i)}\left|W\left(\frac{k}{n}, y\right)-W\left(\frac{k}{n}, \frac{i}{n}\right)\right| \geq \frac{\delta}{4 \sqrt{\varepsilon}}\right)\right] \\
& \leq \sum_{1 \leq k, i \leq n}\left[P\left(\sup _{0 \leq s \leq \frac{1}{n}} \sup _{(x, y) \in[0,1]^{2}}|W(x+s, y)-W(x, y)| \geq \frac{\delta}{4 \sqrt{\varepsilon}}\right)\right. \\
& \left.+P\left(\sup _{0 \leq t \frac{1}{n}}|W(1, t)| \geq \frac{\delta}{4 \sqrt{\varepsilon}} \sqrt{\frac{n}{k}}\right)\right] \\
& \leq C(\mu) n^{3} \exp \left\{-\frac{\delta^{2}}{16 \varepsilon(2+\mu)} n\right\}+C(\mu) n^{2} \exp \left\{-\frac{\delta^{2}}{32 \varepsilon} n\right\} \\
& \leq 2 C(\mu) n^{3} \exp \left\{-\frac{\delta^{2}}{16 \varepsilon(2+\mu)} n\right\} \text {. }
\end{aligned}
$$

Therefore

$$
\limsup _{\varepsilon \rightarrow 0} \varepsilon \log B \leq-\frac{\delta^{2}}{16(2+\mu)} n,
$$

and letting $\mu \rightarrow 0$, we obtain

$$
\limsup _{\varepsilon \rightarrow 0} \varepsilon \log B \leq-\frac{\delta^{2}}{32} n
$$

Combining (5.2.3) with (5.2.4), for $n$ large enough we conclude

$$
\begin{aligned}
& \limsup _{\varepsilon \rightarrow 0} \varepsilon \log (A+B) \leq(\underset{\varepsilon \rightarrow 0}{\limsup } \varepsilon \log A) \bigvee\left(\limsup \varepsilon_{\varepsilon \rightarrow 0} \varepsilon \log B\right) \\
& \leq\left(-l_{\delta}\right) \bigvee\left(-\frac{\delta^{2}}{32} n\right) \\
& \leq-l_{\delta}
\end{aligned}
$$


Noting that $C$ is a closed set. it is easy to show that

$$
\lim _{\delta \rightarrow 0} l_{\delta}=\inf _{f \in C} I(f)
$$

Therefore (5.2.1) is true.

Now we verify (5.2.2). Let $f \in C_{0}[0,1]^{2}$ with $I(f)<\infty$ and $U$ be a neighborhood of $f$. Then for any $\mu>0$ we can find a $g \in U$ and a neighborhood $V$ of $g$ with $V \subset U$ such that $I(g) \leq I(f)+\mu$, and $\frac{\partial^{4} g}{\partial y^{2} \partial x^{2}}$ is a continuous function with bounded variation. Since

$$
P_{\varepsilon}(U) \geq P_{\varepsilon}(V)
$$

it suffices to show that

$$
\liminf _{\Sigma=0} \log P_{\varepsilon}(V) \geq-I(g) \geq-I(f)-\mu
$$

Let $P_{\varepsilon}(g)$ be probability measure on the $C_{0}[0,1]^{2}$ corresponding to $h(x, y)-g(x, y)$, where $h(\cdot, \cdot)$ is distributed according to $P_{\varepsilon}$. Let $G=V-g$. Then

$$
P_{\varepsilon}(V)=P_{\varepsilon}(g)(G)=\int_{G} \frac{d P_{\varepsilon}(g)}{d P_{\varepsilon}} d P_{\varepsilon}
$$

By the Cameron-Martin formula for two variables (cf. Yeh 1963), $P_{\varepsilon}(g)$ is absolutely continuous with respect to $P_{\varepsilon}$ and the Radon-Nikodym derivative $\frac{d P_{\varepsilon}(g)}{d P_{c}}$ is given by

$$
\begin{aligned}
\frac{d P_{\varepsilon}(g)}{d P_{\varepsilon}} & =\exp \left[-\frac{1}{\varepsilon} \int_{[0,1]^{2}} \frac{\partial^{2} g}{\partial y \partial x} d^{2} h-\frac{1}{2 \varepsilon} \int_{0}^{1} \int_{0}^{1}\left(\frac{\partial^{2} g}{\partial y \partial x}\right)^{2} d y d x\right] \\
& =\exp \left[-\frac{1}{\varepsilon} \int_{0}^{1} \int_{0}^{1} h(x, y) \frac{\partial^{4} g}{\partial y^{2} \partial x^{2}} d y d x-\frac{1}{\varepsilon} \frac{\partial^{2} g}{\partial y \partial x}(1,1) h(1,1)-\frac{1}{\varepsilon} I(g)\right] .
\end{aligned}
$$

Let $B_{\delta}=\left\{f \in C_{0}[0,1]^{2}:\|f\|<\delta\right\}$ with $B_{\delta} \subset G$. Noting from (5.2.6) that

$$
\inf _{h \in B_{\delta}}\left(\frac{d P_{\varepsilon}(g)}{d P_{\varepsilon}}\right)(h(\cdot, \cdot)) \geq \exp \left[-\frac{1}{\varepsilon} I(g)-\frac{1}{\varepsilon} \delta\left(\left\|\frac{\partial^{2} g}{\partial y \partial x}\right\|+\left\|\frac{\partial^{4} g}{\partial y^{2} \partial x^{2}}\right\|\right)\right]
$$


and that for any $\delta>0$

$$
P_{\Sigma}\left(B_{\delta}\right) \rightarrow \mathrm{I} \quad \text { as } \quad \varepsilon \rightarrow 0,
$$

we obtain that for positive $\varepsilon$ small enough

$$
\begin{aligned}
P_{\varepsilon}(V) & =P_{\varepsilon}(g)(G) \geq P_{\varepsilon}(g)\left(B_{\delta}\right) \\
& =\int_{B_{\delta}} \frac{d P_{\varepsilon}(g)}{d P_{\xi}} d P_{\Xi} \geq P_{\varepsilon}\left(B_{\delta}\right) \inf _{h \in B_{\delta}}\left(\frac{d P_{\varepsilon}(g)}{d P_{\varepsilon}}\right)(h) \\
& \geq \frac{1}{2} \exp \left[-\frac{1}{\varepsilon} I(g)-\frac{1}{\varepsilon} \delta\left(\left\|\frac{\partial^{2} g}{\partial y \partial x}\right\|+\left\|\frac{\partial^{4} g}{\partial y^{2} \partial x^{2}}\right\|\right)\right] .
\end{aligned}
$$

Thus

$$
\liminf _{\varepsilon \rightarrow 0} \varepsilon \log P_{\varepsilon}(V) \geq-I(g)-\delta\left(\left\|\frac{\partial^{2} g}{\partial y \partial x}\right\|+\left\|\frac{\partial^{4} g}{\partial y^{2} \partial x^{2}}\right\|\right),
$$

and letting $\delta \rightarrow 0$, we have (5.2.5). Therefore the proof of Theorem 5.2.1 is complete.

\subsection{Strassen-Type Laws of the Iterated Logarithm for the Two-Parameter Wiener Process}

Let

$$
K=\left\{f \in C_{0}[0,1]^{2}: I(f) \leq \frac{1}{2}\right\}
$$

Consider the process

$$
\gamma_{v}(x, y)=\frac{W(x, v y)}{\sqrt{2 v \log \log v}}, \quad(0 \leq x, y \leq 1, v \geq 3)
$$

as a function of $v$, taking values in $C_{0}[0,1]^{2}$. Then we have

Theorem 5.3.1 With probability one, the process $\left\{\gamma_{v}(x, y)\right\}$ (as $v \rightarrow \infty$ ) is relatively compact in $C_{0}[0,1]^{2}$, and the set of its limit points is $K$. 
It is easy to verify that

$$
K_{1}=\{f(1, \cdot): f(\cdot, \cdot) \in K\}=\{f(\cdot 1): f(\cdot \cdot \cdot) \in K\}
$$

and hence Theorem 5.3.1 implies the following corollaries.

Corollary 5.3.1 (Strassen 1964). Let. $\{W(t) .0 \leq t \leq \infty\}$ be a standard Wiener process. Then with probability one the process $\left\{\frac{W(v x)}{\sqrt{2 v \log \log v}}, 0 \leq x \leq 1\right\}$ is relatively compact in $C_{0}[0,1]$, and the set of its limit points (as $v \rightarrow \infty$ ) is $K_{1}$.

Corollary 5.3.2 (Wichura 1973). Consider the process

$$
\gamma_{v}(x)=\frac{W(x, v)}{\sqrt{2 v \log \log v}}, \quad 0 \leq x \leq 1, v \geq 3
$$

as a function of $v$ taking values in $C_{0}[0,1]$. Then $\left\{\gamma_{v}(x)\right\}$ (as $v \rightarrow \infty$ ) is relatively compact in $C_{0}[0,1]$, and the set of its limit points is $K_{1}$.

Considering now the process

$$
h_{v}(x, y)=\frac{W(x, v y)}{\sqrt{2 v \log \log v^{-1}}}, \quad 0 \leq x, y \leq 1,0<v \leq \frac{1}{3},
$$

we have

Theorem 5.3.2 With probability one, the process $\left\{h_{v}(x, y)\right\}$ (as $v \rightarrow 0$ ) is relatively compact in $C_{0}[0,1]^{2}$, and the set of its limit points is $K$. 
Corollary 5.3.3 (Gantert 1993). With probability one. the process $\left\{\frac{W(v x)}{\sqrt{2 v \log \log v^{-1}}}, 0 \leq\right.$ $x \leq 1$ ) (as $v \rightarrow 0$ ) is relatively compact in $C_{0}[0,1]$, and the set of its limit points is $K_{1}$.

More generally now; for any $(u, v) \in\left(0, \frac{1}{3}\right]^{2}$. let

$$
\xi_{u, v}(x, y)=\frac{W(u x, v y)}{\sqrt{4 u v \log \log (u v)^{-1}}}, \quad 0 \leq x . y \leq 1 .
$$

Theorem 5.3.3 With probability one, the set of functions $\left\{\xi_{u . v}(\cdot, \cdot): 0<u, v \leq \frac{1}{3}\right\}$ is relatively compact in $C_{0}[0,1]^{2}$ and the set of its limit points coincides with $K$ (as $u . v \rightarrow 0)$.

The following well known law of the iterated logarithm follows from Theorem 5.3.3.

\section{Corollary 5.3.4}

$$
\limsup _{u, v \rightarrow 0} \frac{W(u, v)}{\sqrt{4 u v \log \log (u v)^{-1}}}=1 \quad \text { a.s. }
$$

A special new result is the following theorem, which will be applied to the Kiefer process in Section 5.4.

Theorem 5.3.4 For any $u^{-1}, v \geq 3$, let

$$
\eta_{u, v}(x, y)=\frac{W(u x, v y)}{\sqrt{4 u v \log \log \frac{v}{u}}}, \quad 0 \leq x, y \leq 1
$$

Then, with probability one, the process $\left\{\eta_{u, v}(x, y)\right\}$ (as $u \rightarrow 0, v \rightarrow \infty$ ) is relatively compact in $C_{0}[0,1]^{2}$, and the set of its limit points is $K$. 
Corollary 5.3 .5

$$
\limsup _{u \rightarrow 0, v \rightarrow \infty} \frac{W(u, v)}{\sqrt{4 u v \log \log \frac{v}{u}}}=1 \quad \text { a.s. . }
$$

It is of interest to note that if we replace $u$ by $\frac{1}{v}$ in $\left\{\eta_{u, v}(\cdot . \cdot)\right\}$. then the constant 4 is replaced by 2 . That is to say, we have

Theorem 5.3.5. For any $v \geq 3$, let

$$
\eta_{v}(x, y)=\frac{W\left(\frac{x}{v}, v y\right)}{\sqrt{2 \log \log v}}, \quad 0 \leq x, y \leq 1 .
$$

Then the process $\left\{\eta_{v}(x, y)\right\}$ (as $v \rightarrow \infty$ ) is relatively compact in $C_{0}[0.1]^{2}$ with probatility one. and the set of its limit points is $K$.

\section{Corollary 5.3.6}

$$
\limsup _{v \rightarrow \infty} \frac{W\left(v^{-1}, v\right)}{\sqrt{2 \log \log v}}=1 \quad \text { a.s. }
$$

We only prove Theorem 5.3.1 and Theorem 5.3.3. The proof of Theorem 5.3.2 is similar to that of Theorem 5.3.1, and the proofs of the other theorems are similar to that of Theorem 5.3.3.

Proof of Theorem 5.3.1 For any $\theta>1$, let

$$
v_{j}=\theta^{j}, \quad j=1,2, \cdots
$$


and for any $\delta>0$, let

$$
B(f, \delta)=\left\{g \in C_{0}[0.1]^{2}:\|f-g\|<\delta\right\}, \quad K^{\delta}=\bigcup_{f \in K} B(f, \delta) .
$$

Take $\mu \in(0,1)$ such that

$$
2(1-\mu) \inf _{f \in\left(K^{s}\right)^{c}} I(f)>1 .
$$

Considering $\left\{\gamma_{v_{j}}(\cdot, \cdot)\right\}$, by Theorem 5.2.1 for $j$ large enough we have

$$
\begin{aligned}
P\left(\gamma_{v},(\cdot, \cdot) \in\left(K^{\delta}\right)^{c}\right) & \leq \exp \left[-2(1-\mu) \log \log v_{j} \inf _{f \in\left(K^{\delta}\right)^{c}} I(f)\right] \\
& \leq \mathcal{O}\left(j^{-2(1-\mu) \inf _{f \in\left(K^{\delta}\right)^{c}} I(f)}\right) .
\end{aligned}
$$

Thus

$$
\sum_{j=1}^{\infty} P\left(\gamma_{v}(\cdot, \cdot) \in\left(K^{\delta}\right)^{c}\right)<\infty
$$

By the Borel-Cantelli lemma, we obtain

$$
P\left(\gamma_{v},(\cdot \cdot \cdot) \in\left(K^{\delta}\right)^{c}, \text { i.o. }\right)=0
$$

that is

$$
P\left(\bigcup_{J \geq 1} \bigcap_{j \geq J}\left\{\gamma_{v_{j}}(\cdot, \cdot) \in K^{\delta}\right)=1\right.
$$

Now, for any $v \geq 3$, there exists $j$ such that

$$
v_{j-1} \leq v<v_{j}
$$

Then

$$
\begin{aligned}
\left\|\gamma_{v}-\gamma_{v}\right\| & \leq\left(\sqrt{\frac{v_{j} \log \log v_{j}}{v_{j-1} \log \log v_{j-1}}}-1\right)\left\|\gamma_{v_{j}}\right\|+\left\|\gamma_{v_{j}}\left(\cdot, \frac{v}{v_{j}} \cdot\right)-\gamma_{v_{j}}(\cdot, \cdot)\right\| \\
& :=A(j)+B(j)
\end{aligned}
$$

By (5.3.1), it is easy to show that for $j$ large enough and $\theta$ close enough to 1 ,

$$
A(j)<\delta \quad \text { a.s. }
$$


Noting that, by (5.3.1) for $j$ large enough. we have

$$
\inf _{f \in K}\left\|\gamma_{v_{s}}-f\right\|<\delta \quad \text { a.s. }
$$

as well as

$$
\begin{aligned}
B(j) & \leq 2 \inf _{f \in K}\left\|\gamma_{v_{j}}-f\right\|+\sup _{f \in K}\left\|f\left(\cdot, \frac{v}{v_{j}} \cdot\right)-f(\cdot, \cdot)\right\| \\
& \leq 2 \delta+\sup _{f \in K}\left\|f\left(\cdot, \frac{v}{v_{j}} \cdot\right)-f(\cdot, \cdot)\right\| \quad \text { a.s. }
\end{aligned}
$$

By definition of $K$, for $\theta$ close enough to 1 . we have

$$
\sup _{f \in K}\left\|f\left(\cdot, \frac{v}{v_{j}} \cdot\right)-f(\cdot \cdot)\right\| \leq(\theta-1)^{\frac{1}{2}}<\delta .
$$

Thus, for $j$ large enough and $\theta$ close enough to 1 ,

$$
B(j)<3 \delta \quad \text { a.s. }
$$

Now (5.3.2)and (5.3.3) imply

$$
\left\|\gamma_{v}-\gamma_{v_{j}}\right\|<4 \delta \quad \text { a.s. }
$$

Therefore, combining (5.3.1)and (5.3.4), we obtain

$$
P\left(\bigcup_{V>0} \bigcap_{v \geq V}\left\{\gamma_{v}(\cdot, \cdot) \in K^{5 \delta}\right)=1 .\right.
$$

On the other hand, for any $f \in K$, we want to show that

$$
\liminf _{j \rightarrow \infty}\left\|\gamma_{v_{j}}-f\right\|=0 \quad \text { a.s. }
$$

Let

$$
\gamma_{j}^{*}(x, y)= \begin{cases}0, & (x, y) \in[0,1]^{2}-\left[\frac{1}{\theta}, 1\right]^{2} \\ \frac{W\left(x, v_{j} y\right)-W\left(x, v_{j-1}\right)-W\left(\frac{1}{\theta}, v_{j} y\right)+W\left(\frac{1}{\theta}, v_{j-1}\right)}{\sqrt{2 v_{j} \log \log v_{j}}}, & (x, y) \in\left[\frac{1}{\theta}, 1\right]^{2}\end{cases}
$$


and

$$
f_{\theta}(x, y)= \begin{cases}0, & (x, y) \in[0,1]^{2}-\left[\frac{1}{\theta}, 1\right]^{2}, \\ f(x, y)-f\left(x, \theta^{-1}\right)-f\left(\theta^{-1}, y\right)+f\left(\theta^{-1} \cdot \theta^{-1}\right), & (x, y) \in\left[\theta^{-1}, 1\right]^{2} .\end{cases}
$$

In the light of (5.3.5) and continuity of $f(x, y)$. (5.3.6) will be shown if we can prove that

$$
\liminf _{j \rightarrow \infty}\left\|\gamma_{j}^{*}-f_{\theta}\right\|=0 \quad \text { a.s. }
$$

For any $\delta>0$ and $\mu>0$, by Remark 5.2 .1 , for $j$ large enough we have

$$
\begin{aligned}
P\left(\left\|\gamma_{j}^{*}-f_{\theta}\right\|<\delta\right) & =P\left(\sup _{(x, y) \in\left[0,1-\theta^{-1}\right]^{2}}\left|\gamma_{j}^{*}\left(x+\theta^{-1}: y+\theta^{-1}\right)-f_{\theta}\left(x+\theta^{-1}, y+\theta^{-1}\right)\right|<\delta\right) \\
& \geq \exp \left[-(1+\mu) \log \log v_{j} \inf _{g \in B\left(f_{\theta} . \delta\right)} \int_{0}^{1-\theta^{-1}} \int_{0}^{1-\theta^{-1}}\left(\frac{\partial^{2} g}{\partial y \partial x}\right)^{2} d x d y\right] \\
& \geq C j^{-(1+\mu) \inf _{g \in B\left(f_{\theta}, \delta\right)} \int_{0}^{1-\theta^{-1}} \int_{0}^{1-\theta^{-1}}\left(\frac{\partial^{2} g}{\partial y \partial x}\right)^{2} d x d y}
\end{aligned}
$$

where $C$ is some positive constant. Since

$$
\int_{0}^{1-\frac{1}{\theta}} \int_{0}^{1-\frac{1}{\theta}}\left(\frac{\partial^{2} f_{\theta}}{\partial y \partial x}\right)^{2} d x d y \leq \int_{0}^{1-\frac{1}{\theta}} \int_{0}^{1-\frac{1}{\theta}}\left(\frac{\partial^{2} f}{\partial y \partial x}\right)^{2} d x d y \leq 1
$$

we have

$$
\inf _{g \in B\left(f_{\theta}, \delta\right)} \int_{0}^{1-\frac{1}{\theta}} \int_{0}^{1-\frac{1}{\theta}}\left(\frac{\partial^{2} g}{\partial y \partial x}\right)^{2} d x d y<1 .
$$

Therefore, we can take $\mu>0$ small enough so that

$$
\sum_{j=1}^{\infty} P\left(\left\|\gamma_{j}^{*}-f_{\theta}\right\|<\delta\right)=\infty
$$

Noting that the random variables $\left\{\gamma_{j}^{*}(\cdot, \cdot)\right\}$ are independent, via the Borel-Cantelli lemma we obtain (5.3.7). The proof of Theorem 5.3.1 is now complete.

Proof of Theorem 5.3.2 Firstly, we have to show that the set of limit pionts for $\left\{\xi_{u, v}(\cdot, \cdot): 0<u, v \leq \frac{1}{3}\right\}$ is contained in $K$. It suffices to show that for any $\delta>0$

$$
P\left(\bigcup_{U>0} \bigcup_{V>0} \bigcap_{0<u<U} \bigcap_{0<v<V}\left\{\xi_{u, v}(\cdot, \cdot) \in K^{5 \delta}\right\}\right)=1
$$


where $K^{-5 \delta}:=\bigcup_{f \in K} B(f, 5 \delta)$, and $B(f, 5 \delta):=\left\{h \in C_{0}[0,1]^{2}:\|h-f\|<5 \delta\right\}$.

For $\theta>1$, define

$$
u_{k}=\theta^{-k}, \quad k=1,2, \cdots
$$

and

$$
v_{j}=\theta^{-j}, \quad j=1,2, \cdots
$$

Now. we choose $\mu \in(0,1)$ such that

$$
2(1-\mu) \inf _{f \in\left(K^{\delta}\right)^{c}} I(f)>1 .
$$

Considering $\left\{\xi_{u_{k}, v_{j}}(\cdot, \cdot)\right\}$, by Theorem 5.2.1, for $k$ and $j$ large enough we have

$$
\begin{aligned}
P\left(\xi_{u_{k}, v_{j}}(\cdot, \cdot) \in\left(K^{\delta}\right)^{c}\right) & \leq \exp \left\{-4(1-\mu) \log \log \left(u_{k} v_{j}\right)^{-1} \inf _{f \in\left(K^{\delta}\right)^{c}} I(f)\right\} \\
& \leq \mathcal{O}\left((k+j)^{-4(1-\mu) \inf _{f \in\left(K^{\delta}\right)^{c}} I(f)}\right) .
\end{aligned}
$$

Thus

$$
\sum_{k=1}^{\infty} \sum_{j=1}^{\infty} P\left(\xi_{u_{k}, v_{j}}(\cdot, \cdot) \in\left(K^{\delta}\right)^{c}\right)<\infty
$$

Therefore by the Borel-Cantelli lemma

$$
P\left(\bigcup_{K \geq 1} \bigcup_{J \geq 1} \bigcap_{k \geq K} \bigcap_{j \geq J}\left\{\xi_{u_{k}, v_{j}}(\cdot, \cdot) \in K^{-\delta}\right\}\right)=1 .
$$

Since for any $u, v \in(0,1]$, there exist $k, j \in N$, such that

$$
u_{k+1}<u \leq u_{k}, \quad v_{j+1}<v \leq v_{j}
$$

we obtain

$$
\begin{aligned}
\left\|\xi_{u, v}(\cdot, \cdot)-\xi_{u_{k}, v_{j}}(\cdot, \cdot)\right\| \leq & \left\|\xi_{u_{k}, v_{j}}\left(\frac{u}{u_{k}} \cdot \frac{v}{v_{j}} \cdot\right)-\xi_{u_{k}, v_{j}}(\cdot, \cdot)\right\| \\
& +\left(\sqrt{\frac{u_{k} v_{j} \log \log \left(u_{k} v_{j}\right)^{-1}}{u_{k+1} v_{j+1} \log \log \left(u_{k+1} v_{j+1}\right)^{-1}}}-1\right)\left\|\xi_{u_{k}, v_{j}}(\cdot, \cdot)\right\| \\
:= & C(k, j)+D(k, j) .
\end{aligned}
$$


Via (5.3.9). it is easy to show that for $\theta$ close enough to 1 and $k . j$ large enorgh,

$$
D(k, j)<\delta \quad \text { a.s. }
$$

We want to show that for $k, j$ large enough and $\theta$ close enough to 1 . we have

$$
C(k, j)<3 \delta \quad \text { a.s. }
$$

Since, by (5.3.9), for $k, j$ large enough, we have

$$
\inf _{f \in K}\left\|\xi_{u_{k}, v_{j}}-f\right\|<\delta \quad \text { a.s. }
$$

as well as

$$
\begin{aligned}
C(k \cdot j) & \leq 2 \inf _{f \in K}\left\|\xi_{u_{k}, v_{j}}(\cdot, \cdot)-f(\cdot, \cdot)\right\|+\sup _{f \in K}\left\|f\left(\frac{u}{u_{k}}, \frac{v}{v_{j}} \cdot\right)-f(\cdot \cdot)\right\| \\
& \leq 2 \delta+\sup _{f \in K}\left\|f\left(\frac{u}{u_{k}} \cdot \frac{v}{v_{j}} \cdot\right)\right\| \quad \text { a.s. }
\end{aligned}
$$

By the definition of $K$, we can take $\theta>1$ (close enough to 1 ) such that

$$
\sup _{f \in K}\left\|f\left(\frac{u}{u_{k}} \cdot \frac{v}{v_{j}} \cdot\right)-f(\cdot, \cdot)\right\|<\delta .
$$

Thus, for $k, j$ large enough,

$$
C(k, j)<3 \delta \quad \text { a.s. }
$$

Therefore, for $k, j$ large enough,

$$
\left\|\xi_{u, v}(\cdot, \cdot)-\xi_{u_{k}, v_{j}}(\cdot, \cdot)\right\|<4 \delta \quad \text { a.s. }
$$

Combining (5.3.9) with (5.3.10), we obtain (5.3.8).

For completing the proof, we have to show that for any $g \in K$,

$$
\liminf _{k, j \rightarrow \infty}\left\|\xi_{u_{k}, v_{j}}(\cdot, \cdot)-g(\cdot, \cdot)\right\|=0 \quad \text { a.s. }
$$


Define

$\tilde{\xi}_{k, j}(x, y)= \begin{cases}0, & (x, y) \in[0.1]^{2}-\left[\frac{1}{\theta} \cdot 1\right]^{2} . \\ \frac{W\left(u_{k} x . v_{j} y\right)-W\left(u_{k} x, v_{j+1}\right)-W\left(u_{k+1}, v_{j} y\right)+W\left(u_{k+1}, v_{j+1}\right)}{\sqrt{4 u_{k}, v_{j} \log \log \left(u_{k} v_{j}\right)^{-1}}}, & (x, y) \in\left[\frac{1}{\theta}, 1\right]^{2} .\end{cases}$

and

$$
g_{\theta}(x, y)= \begin{cases}0, & (x, y) \in[0,1]^{2}-\left[\frac{1}{\theta}, 1\right]^{2} . \\ g(x, y)-g\left(x \cdot \frac{1}{\theta}\right)-g\left(\frac{1}{\theta}, y\right)+g\left(\frac{1}{\theta}, \frac{1}{\theta}\right), & (x, y) \in\left[\frac{1}{\theta}, 1\right]^{2}\end{cases}
$$

By (5.3.8) and continuity of $g(x, y),(5.3 .11)$ will be shown if we can prove that

$$
\liminf _{k, j \rightarrow \infty}\left\|\bar{\xi}_{k, j}(\cdot, \cdot)-g_{\theta}(\cdot, \cdot)\right\|=0 \quad \text { a.s. }
$$

For any $\delta>0, \mu>0$, by Theorem 5.2 .1 and Remark 5.2.1, we have

$$
\begin{aligned}
& P\left(\left\|\tilde{\xi}_{k, j}(\cdot, \cdot)-g_{\theta}(\cdot, \cdot)\right\|<\delta\right)= \\
& \quad P\left(\sup _{(x, y) \in\left[0,1-\frac{1}{\theta}\right]^{2}}\left|\tilde{\xi}_{k, j}\left(x+\frac{1}{\theta}, y+\frac{1}{\theta}\right)-g_{\theta}\left(x+\frac{1}{\theta}, y+\frac{1}{\theta}\right)\right|<\delta\right) \\
& \quad \geq \exp \left[-2(1+\mu) \log \log \left(u_{k} v_{j}\right)^{-1} \inf _{f \in B\left(g_{\theta}, \delta\right)} \int_{0}^{1-\frac{1}{\theta}} \int_{0}^{1-\frac{1}{\theta}}\left(\frac{\partial^{2} f}{\partial y \partial x}\right)^{2} d x d y\right] \\
& \quad \geq C(k+j)^{-2(1+\mu) \text { inf }_{f \in B\left(g_{\theta}, \delta\right)} \int_{0}^{1-\frac{1}{\theta}} \int_{0}^{1-\frac{1}{\theta}}\left(\frac{\partial^{2} f}{\partial y \partial x}\right)^{2} d x d y},
\end{aligned}
$$

where $C$ is some positive constant. Since

$$
\int_{0}^{1-\frac{1}{\theta}} \int_{0}^{1-\frac{1}{\theta}}\left(\frac{\partial^{2} g_{\theta}}{\partial y \partial x}\right)^{2} d x d y \leq \int_{0}^{1} \int_{0}^{1}\left(\frac{\partial^{2} g}{\partial y \partial x}\right)^{2} d x d y \leq 1
$$

hence

$$
\inf _{f \in B\left(g_{\theta}, \delta\right)} \int_{0}^{1-\frac{1}{\theta}} \int_{0}^{1-\frac{1}{\theta}}\left(\frac{\partial^{2} f}{\partial y \partial x}\right)^{2} d x d y<1
$$

Therefore, we can take $\mu>0$ small enough such that

$$
\sum_{k=1}^{\infty} \sum_{j=1}^{\infty} P\left(\left\|\bar{\xi}_{k, j}(\cdot, \cdot)-g_{\theta}(\cdot, \cdot)\right\|<\delta\right)=\infty
$$


Noing that $\left\{\tilde{\xi}_{k, j}(\cdot, \cdot)\right\}$ are independent. via the Borel-Cantelli lemma we obtain (5.3.12).

The proof of Theorem 5.3.3 is now complete.

\subsection{Strassen-Type Laws of the Iterated Logarithm for the Kiefer Process and the Empirical Pro- cess}

Let $\left\{W(x, y),(x, y) \in[0, \infty)^{2}\right\}$ be a two-parameter Wiener process. A Kiefer process

$$
\{K(x, y), 0 \leq x \leq 1,0 \leq y<\infty\}
$$

is defined by

$$
K(x, y)=W(x, y)-x W(1, y)
$$

The results of Section 5.3 can be extended to the Kiefer process. For $v \geq 3$, let

$$
J_{v}(x, y)=\frac{K(x, v y)}{\sqrt{2 v \log \log v}}, \quad 0 \leq x, y \leq 1
$$

We have the following result.

Theorem 5.4.1 With probability one, the process $\left\{J_{v}(x, y)\right\}($ as $v \rightarrow \infty)$ is relatively compact in $C_{0}[0,1]^{2}$, and the set of its limit points is $G$ where

$$
G=\{g \in K: g(1, y)=0, \text { for any } y \in[0,1]\} \text {. }
$$


This theorem implies

Corollary 5.4.1 (Csörgö-Révész 1981). Consider the process

$$
J_{v}(x)=\frac{K\left(x, v^{\prime}\right)}{\sqrt{2 v \log \log v^{\prime}}} \quad(0 \leq x \leq 1, v \geq 3)
$$

as a function of $v$ taking values in $C_{0}[0,1]$. Then $\left\{J_{v}(x)\right\}$ (as $v \rightarrow \infty$ ) is relatively compact in $C_{0}[0,1]$, and the set of its limit points is $\mathcal{F}$ where $\mathcal{F} \subset K_{1}$ is the set of functions $f$ for which $f(1)=0$.

Remark 5.4.1 Via Corollary 5.4.1 and the Kiefer-type Komlós-Major-Tusnády strong approximation of the empirical process (cf. Komlós-Major-Tusnády 1975; CsörgöRévész 1981, Theorem 4.4.3), we can obtain the corresponding result for empirical processes that is due to Finkelstein(1971).

Proof of Theorem 5.4.1 Firstly, it is easy to verify that

$$
G=\{f(x, y)-x f(1, y): f \in K\}
$$

By Theorem 5.3.1, with probability one, for any sequence $\left\{v_{n}\right\}$ with $\lim _{n \rightarrow \infty} v_{n}=\infty$, there exists a subsequence $\left\{v_{n_{k}}\right\}$ and $f \in K$ such that

$$
\left\|\gamma_{v_{n_{k}}}(\cdot, \cdot)-f(\cdot, \cdot)\right\| \rightarrow 0, \quad(k \rightarrow \infty)
$$

On noting

$$
\begin{aligned}
& \sup _{(x, y) \in[0,1]^{2}}\left|J_{v_{n_{k}}}(x, y)-(f(x, y)-x f(1, y))\right| \\
& \quad \leq \sup _{(x, y) \in[0,1]^{2}}\left|\gamma_{v_{n_{k}}}(x, y)-f(x, y)\right|+\sup _{(x, y) \in[0,1]}\left|\gamma_{v_{n_{k}}}(1, y)-f(1, y)\right|
\end{aligned}
$$


and taking $g(x . y)=f(x, y)-x f(1, y)$, we have

$$
\left\|J_{v_{n_{k}}}(\cdot, \cdot)-g(\cdot, \cdot)\right\| \rightarrow 0, \quad(k \rightarrow \infty)
$$

Therefore. with probability one, $\left\{J_{v}(\cdot, \cdot), v \geq 3\right\}$ is relatively compact, and the set of its limit points is contained in $G$.

On the other hand, for any $g \in G$, there exists $f \in K$ such that

$$
g(x, y)=f(x, y)-x f(1, y), \quad(x, y) \in[0.1]^{2} .
$$

Hence, via Theorem 5.3.1, there exists $\left\{v_{n}^{\prime}\right\}$ with $\lim _{n \rightarrow \infty} v_{n}^{\prime}=\propto$ such that

$$
\left\|\gamma_{v_{n}^{\prime}}(\cdot, \cdot)-f(\cdot, \cdot)\right\| \rightarrow 0, \quad(k \rightarrow \infty)
$$

Thus

$$
\left\|J_{v_{n}^{\prime}}(\cdot, \cdot)-g(\cdot, \cdot)\right\| \rightarrow 0, \quad(k \rightarrow \infty)
$$

Therefore $g$ is a limit point of $\left\{J_{v}(\cdot, \cdot), v \geq 3\right\}$. The proof of Theorem 5.4.1 is now complete.

Now, for any $u^{-1}, v \geq 3$, let

$$
G_{u, v}(x, y)=\frac{K(u x, v y)}{\sqrt{4 u v \log \log \frac{v}{u}}}, \quad 0 \leq x, y \leq 1 .
$$

Then, by Theorem 5.3.5, a natural result is the following theorem.

Theorem 5.4.2. With probability one, the process $\left\{G_{u, v}(x, y)\right\}$ (as $u \rightarrow 0, v \rightarrow \infty$ ) is relatively compact in $C_{0}[0,1]^{2}$, and the set of its limit points is $K$. 
This theorem means that the two-parameter Wiener process and the Kiefer process have the same path properties along the $y$-axis.

Corollary 5.4.2. With probability one, the process $\left\{G_{u, v}(x, 1)\right\}$ (as $u \rightarrow 0 . v \rightarrow \infty$ ) is relatively compact in $C_{0}[0,1]$, and the set of its limit points is $K_{1}$.

Corollary 5.4 .3

$$
\limsup _{u \rightarrow 0, v \rightarrow \infty} \frac{K(u, v)}{\sqrt{4 u v \log \log \frac{v}{u}}}=1 \quad \text { a.s. }
$$

Let $X_{1}, X_{2}, \cdots$ be i.i.d.r.v. with distribution function $F$, and define the empirical distribution function of the sample $X_{1}, X_{2}, \cdots, X_{n}$ by

$$
F_{n}(x)=\frac{1}{n} \sum_{i=1}^{n} I_{(-\infty, x]}\left(X_{i}\right) \text {. }
$$

By the Kiefer-type Komlós-Major-Tusnády strong approximation of the empirical process (cf. Komlós-Major-Tusnády 1975; Csörgö-Révész 1981, Theorem 4.4.3), the following result can be obtained.

Theorem 5.4.3 Let $F$ be a continuous distribution function over the real line. Then the process

$$
\beta_{u, n}(x)=\left(\frac{n}{4 u \log \log \frac{n}{u}}\right)^{\frac{1}{2}}\left(F_{n}\left(F^{-1}(u x)\right)-u x\right), \quad 0 \leq x \leq 1,
$$

is relatively compact with probability one (as $u \rightarrow 0, n \rightarrow \infty$ ), and the set of its limit points (with respect to the sup norm) is $K_{1}$. 
Theorem 5.4.3 immediately implies

Corollary 5.4.4 Under assumptions of Theorem 5.4.3

$$
\limsup _{u \rightarrow 0 . n \rightarrow \infty}\left(\frac{n}{4 u \log \log \frac{n}{u}}\right)^{\frac{1}{2}}\left(F_{n}\left(F^{-1}(u)\right)-u\right)=1 \quad \text { a.s. }
$$




\section{Chapter 6}

\section{Large Deviations for Two-Parameter Gaussian Processes Related to Change-Point Analysis}

\subsection{Introduction}

Let $F(x)$ be a non-degenerate distribution function. We say that the separable real valued process $K:=\left\{K_{F}(x, t), x \in R, 0 \leq t<\infty\right\}$ is a Kiefer process indexed by $F$, if $K_{F}$ is a Gaussian process with zero mean and covariance

$$
E K_{F}(x, t) K_{F}(y, s)=(F(x \wedge y)-F(x) F(y))(t \wedge s)
$$

for all $x, y \in R$ and $0 \leq t, s<\infty$. We say that the separable real valued process $\Gamma:=\left\{\Gamma_{F}(x, t), x \in R, 0 \leq t \leq 1\right\}$ is a "tied down" Kiefer process indexed by $F$, if $\Gamma_{F}$ is a Gaussian process with $E \Gamma_{F}(x, t)=0$ and

$$
E \Gamma_{F}(x, t) \Gamma_{F}(y: s)=\{F(x \wedge y)-F(x) F(y)\}(t \wedge s-t s)
$$

for all $x, y \in R$ and $0 \leq t, s \leq 1$. Let $X_{1} \cdots X_{n} \cdots$ be independent random variables. A natural way to test for the possibility of having a change in the distribution of such a sequence of chronologically observed data up to time $n$ is to compare their respective empirical distributions before and after the possibly unknown time 
and

$$
\sup _{0<t<1} \sup _{x \in R}\left|\hat{\beta}_{n}(x, t)\right| / \hat{q}(t) \stackrel{D}{\rightarrow} \sup _{0<t<1} \sup _{x \in R}\left|\Gamma_{F}(r, t)\right| / \hat{q}(t)
$$

The respective distributions of the latter two random variables are not known even in the case of $q(t)=\hat{q}=1$. Hence estimating their distributions is of practical, as well as of theoretical interest. This leads us to studying the tail behaviour of the distributions of $\sup _{0<t \leq 1} \sup _{x \in R}\left|K_{F}(x, t)\right| / q(t)$ and $\sup _{0<t<1} \sup _{x \in R}\left|\Gamma_{F}(x, t)\right| / \hat{q}(t)$. Naturally enough, our work is based on sample path large deviations for appropriate two-parameter Gaussian processes in weighted metric. The desired tail probability estimates will follow as a consequence of such investigations.

Let $E$ be a metric space. We say that a function $I: E \rightarrow[0, \infty]$ is a rate function, if $I$ is lower semicontinuous. We say that a rate function $I: E \rightarrow[0, \infty]$ is good, if $\{x \in E: I(x) \leq a\}$ is compact for each $a \geq 0$. Let $\left\{\theta_{\varepsilon}: \varepsilon>0\right\}$ be a family of probability measures on $E$. We say that $\left\{\theta_{\varepsilon}, \varepsilon>0\right\}$ satisfies the large deviation principle (LDP) with a rate function $I$, if it satisfies the following conditions :

a) for any open set $G \subset E$,

$$
\liminf _{\varepsilon \rightarrow 0} \varepsilon \log \theta_{\varepsilon}(G) \geq-\inf _{x \in G} I(x)
$$

b) for any closed set $H \subset E$,

$$
\limsup _{\varepsilon \rightarrow 0} \varepsilon \log \theta_{\varepsilon}(H) \leq-\inf _{x \in H} I(x)
$$

In Section 6.2, we prove a LDP for a Brownian sheet in $q$-metric equipped $C_{0}[0,1]^{2}$, which is an extension of that in the usual sup-norm equipped $C_{0}[0,1]^{2}$. We extend 
the results of Section 6.2 to Kiefer and "tied down" Kiefer processes in Section 6.3 and 6.4, respectively, and then obtain probability estimates for their tails in $q$-metric.

\subsection{Large deviations for Brownian sheet in q-metric}

Let $C_{0}[0,1]^{2}$ be the space of continuous functions on $[0,1]^{2}$ which vanish along the axes. Let $W:=\{W(x, t), 0 \leq x, t \leq 1\}$ be a standard Brownian sheet. that is a Gaussian process on $[0,1]^{2}$ with zero mean and covariance function

$$
E W\left(x_{1}, t_{1}\right) W\left(x_{2}, t_{2}\right)=\left(x_{1} \wedge x_{2}\right)\left(t_{1} \wedge t_{2}\right)
$$

Throughout this paper, we assume that $q(t)$ is a monotone increasing function on $[0,1]$, which is strictly positive for $t>0$, and satisfies the following condition:

$$
J(q, c)<\infty, \text { for some } c>0,
$$

where

$$
J(q, c)=\int_{0}^{1} \frac{1}{t} \exp \left\{-c \frac{q^{2}(t)}{t}\right\} d t
$$

Define the $q$-metric on $C_{0}[0,1]^{2}$ by

$$
d_{q}(f, g):=\sup _{0 \leq x \leq 1} \sup _{0<t \leq 1}\{|f(x, t)-g(x, t)| / q(t)\}
$$

for $f, g \in C_{0}[0,1]^{2}$. For any $\varepsilon>0$, let $P_{\varepsilon}$ be a probability measure on $C_{0}[0,1]^{2}$ corresponding to $\sqrt{\varepsilon} W$. 
Theorem 6.2.1 The net of probability measures $\left\{P_{\varepsilon}, \varepsilon>0\right\}$ satisfies the large deviation principle in q-metric equipped $C_{0}[0,1]^{2}$ with the good rate function $I: C_{0}[0.1]^{2} \rightarrow$ $[0, \infty]$ that is defined by

$$
I(f)= \begin{cases}\int_{0}^{1} \int_{0}^{1} \frac{\partial^{2} f(x, t)}{\partial x \partial t} d x d t . & \text { if } f \text { is an absolutely continuous function } \\ & \text { with square integrable } \frac{\partial^{2} f}{\partial x \partial t} \\ \infty, & \text { otherwise. }\end{cases}
$$

Remark 6.2.1 Via Theorem 6.2.1, it is easy to obtain a similar result for a standard Brownian motion (one parameter), which is due to Beibel and Lerche(1994).

\section{Corollary 6.2.1}

$$
\lim _{u \rightarrow \infty} \frac{\log P\left(\|W\|_{q} \geq u\right)}{u^{2}}=-\inf _{0<t \leq 1} \frac{q^{2}(t)}{2 t}
$$

Lemma 6.2.1 (Pruitt.Orey 1973) Let $0 \leq x_{1}<x_{2}, 0 \leq y_{1}<y_{2}$. Then for any $u>0$

$$
\begin{aligned}
& P\left(\sup _{x_{1} \leq s_{1}<s_{2} \leq x_{2}, y_{1} \leq t_{1}<t_{2} \leq y_{2}}\left|W\left(s_{2}, t_{2}\right)-W\left(s_{2}, t_{1}\right)-W\left(s_{1}, t_{2}\right)+W\left(s_{1}, t_{1}\right)\right| \geq u\right) \\
& \leq 4 P\left(\left|W\left(x_{2}, y_{2}\right)-W\left(x_{2}, y_{1}\right)-W\left(x_{1}, y_{2}\right)+W\left(x_{1}, y_{1}\right)\right| \geq u\right) .
\end{aligned}
$$

Lemma 6.2.2 For any $b \in(0,1)$ and a large enough, we have

$$
P\left(\sup _{0<t \leq b, 0 \leq x \leq 1} \frac{|W V(x, t)|}{q(t)} \geq a\right) \leq C J\left(q, \frac{a^{2}}{8}\right) \exp \left\{-\frac{a^{2}}{8} \inf _{0<t \leq b} \frac{q^{2}(t)}{2 t}\right\} .
$$

Proof of Lemma 6.2.2 By Lemma 6.2.1, we have

$$
\begin{aligned}
& P\left(\sup _{0<t \leq b, 0 \leq x \leq 1} \frac{|W(x, t)|}{q(t)} \geq a\right) \\
& \leq \sum_{k=1}^{\infty} P\left(\sup _{0<t \leq b 2^{-k+1}, 0 \leq x \leq 1}|W(x, t)| \geq a q\left(2^{-k}\right)\right) \\
& \leq 4 \sum_{k=1}^{\infty} P\left(\left|W\left(1, b 2^{-k+1}\right)\right| \geq a q\left(b 2^{-k}\right)\right) \\
& \leq C \sum_{k=1}^{\infty} \exp \left\{-\frac{a^{2} q^{2}\left(b 2^{-k}\right)}{8 b 2^{-k}}\right\} \\
& \leq C \exp \left\{-\frac{a^{2}}{8} \inf _{0<t \leq b} \frac{q^{2}(t)}{2 t}\right\} J\left(q, \frac{a^{2}}{8}\right) .
\end{aligned}
$$

Hence, this lemma is proved. 
where

$$
\begin{aligned}
& a_{n}(k, i)=n^{2}\left[f\left(\frac{k+1}{n}, \frac{i+1}{n}\right)-f\left(\frac{k+1}{n} \cdot \frac{i}{n}\right)-f\left(\frac{k}{n}, \frac{i+1}{n}\right)+f\left(\frac{k}{n}, \frac{i}{n}\right)\right], \\
& I_{n}(k, i)=\left[\frac{k}{n}, \frac{k+1}{n}\right] x\left[\frac{i}{n}, \frac{i+1}{n}\right] .
\end{aligned}
$$

and $b_{n}(k, i), c_{n}(k, i)$ and $d_{n}(k, i)$ are constants such that

$f(x, t)=f_{n}(x, t),(x, t) \in\left\{\left(\frac{k}{n}, \frac{i}{n}\right),\left(\frac{k+1}{n}, \frac{i}{n}\right),\left(\frac{k}{n}, \frac{i+1}{n}\right),\left(\frac{k+1}{n}, \frac{i+1}{n}\right)\right\}, 0 \leq k, i \leq n$.

For any $\delta>0$, let $H^{\delta}:=\left\{f: \inf _{g \in H}\|f-g\|_{q}<\delta\right\}$. Then

$$
\begin{aligned}
P_{\varepsilon}(H) & \leq P_{\varepsilon}\left(f \mid f_{n} \in H^{\delta}\right)+P_{\varepsilon}\left(f \mid\left\|f_{n}-f\right\|_{q} \geq \delta\right) \\
& :=A+B
\end{aligned}
$$

Let $l_{\delta}:=\inf _{f \in H^{\delta}} I(f)$. Since $\frac{1}{\varepsilon n^{2}} \sum_{k=1}^{n} \sum_{i=1}^{n} a_{n}^{2}(k, i)$ is a $\chi_{n^{2}}^{2}$ random variable under $P_{\varepsilon}$, hence

$$
\begin{aligned}
A & \leq P_{\varepsilon}\left(f \mid I\left(f_{n}\right) \geq l_{\delta}\right) \\
& \leq \frac{1}{\Gamma\left(\frac{n^{2}}{2}\right) 2^{\frac{n^{2}}{2}}} \int_{\frac{2 l_{\delta}}{\varepsilon}}^{\infty} \exp \left\{-\frac{x}{2}\right\} x^{\frac{n^{2}}{2}-1} d x \\
& \leq C(n) \exp \left\{-\frac{(1-\mu)}{\xi} l_{\delta}\right\},
\end{aligned}
$$

where $C(n)$ is a constant that depends on $n$, and $\mu$ is any positive number. Therefore,

$$
\limsup _{\varepsilon \rightarrow 0} \varepsilon \log A \leq-l_{\delta}+\mu l_{\delta},
$$

and letting $\mu \rightarrow 0$, we obtain

$$
\limsup _{\varepsilon \rightarrow 0} \varepsilon \log A \leq-l_{\delta}
$$


Therefore. we get

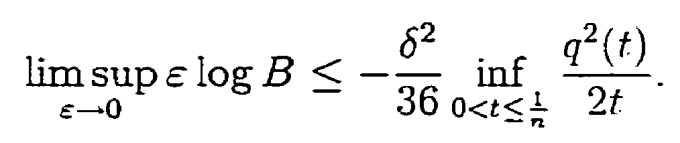

Noting that $\lim _{t \downarrow 0} \frac{q^{2}(t)}{2 t}=\infty$, and combining (6.2.2) with (6.2.3), for $n$ large enough, we have

$$
\limsup _{\varepsilon \rightarrow 0} \varepsilon \log (A+B) \leq-l_{\delta}
$$

Since $H$ is a closed set, by Lemma 6.2.4, letting $\delta \downarrow 0$, we obtain (6.2.1) from (6.2.4).

For completing the proof, we have to show that for any open set $G \subset C_{0}[0,1]^{2}$,

$$
\liminf _{\varepsilon \rightarrow 0} \varepsilon \log P_{\varepsilon}(G) \geq-\inf _{f \in G} I(f)
$$

Let $f \in G$ with $I(f)<\infty$. Then there exists $\delta>0$ such that

$$
B_{q}(f, \delta):=\left\{g:\|f-g\|_{q}<\delta\right\} \subset G
$$

Let $P_{\varepsilon}(f)$ be the probability measure on the $C_{0}[0,1]^{2}$ corresponding to $h(x, t)-f(x, t)$, where $h(x, t)$ is distributed according to $P_{\varepsilon}$. Then, via the Cameron-Martin formula (cf. Bass,1988; or J. Yeh,1963) and Jensen's inequality, we have

$$
\begin{aligned}
P_{\varepsilon}\left(B_{q}(f . g)\right) & =P_{\varepsilon}(f)\left(B_{q}(\delta)\right) \\
& =\int_{B_{q}(\delta)} \frac{d P_{\varepsilon}(\delta)}{d P_{\varepsilon}}(h) P_{\varepsilon}(d h) \\
& =\int_{B_{q}(\delta)} \exp \left\{\frac{1}{\varepsilon} \int_{[0,1]^{2}} \frac{\partial^{2} f}{\partial x \partial y} d h-\frac{1}{\varepsilon} I(f)\right\} P_{\varepsilon}(d h) \\
& =\exp \left\{-\frac{1}{\varepsilon} I(f)\right\} P_{\varepsilon}\left(B_{q}(\delta)\right) \cdot \frac{1}{P_{\varepsilon}\left(B_{q}(\delta)\right)} \int_{B_{q}(\delta)} \exp \left\{\frac{1}{\varepsilon} \int_{[0,1]^{2}} \frac{\partial^{2} f}{\partial x \partial t} d h\right\} P_{\varepsilon}(d h) \\
& \geq \exp \left\{-\frac{1}{\varepsilon} I(f)\right\} P_{\varepsilon}\left(B_{q}(\delta) \exp \left\{\frac{1}{\varepsilon P_{\varepsilon}\left(B_{q}(\delta)\right)} \int_{B_{q}(\delta)} \int_{[0,1]^{2}} \frac{\partial^{2} f}{\partial x \partial t} d h P_{\varepsilon}(d h)\right\}\right. \\
& =\exp \left\{-\frac{1}{\varepsilon} I(f)\right\} P_{\varepsilon}\left(B_{q}(\delta)\right) .
\end{aligned}
$$


Since. by Lemma 6.2.2. we have

$$
\lim _{\varepsilon \rightarrow 0} P_{\varepsilon}\left(B_{q}(\delta)\right)=1
$$

we obtain

$$
\liminf _{\xi \rightarrow 0} \bumpeq \log P_{\varepsilon}\left(B_{q}(f, \delta)\right) \geq-I(f) .
$$

Therefore (6.2.5) is true. and the proof of Theorem 6.2.1 is complete.

Proof of Corollary 6.2.1 Let $H_{1}=\left\{f \in C_{0}[0,1]^{2}:\|f\|_{q} \geq 1\right\}$, and $G_{1}=\{f \in$ $\left.C_{0}[0,1]^{2}:\|f\|_{q}>1\right\}$. Then we have to show that

$$
\inf _{f \in H_{1}} I(f) \leq \inf _{0<t \leq 1} \frac{q^{2}(t)}{2 t} \leq \inf _{f \in G_{1}} I(f)
$$

Since, for $f \in H_{1}$ with $I(f)<\infty$,

$$
\frac{|f(x, t)|^{2}}{q^{2}(t)} \leq I(f) \frac{2 t}{q^{2}(t)}
$$

for any $(x, t) \in[0.1] \times(0,1]$, hence we have

$$
\inf _{f \in H_{1}} I(f) \geq \inf _{0<t \leq 1} \frac{q^{2}(t)}{2 t} .
$$

On the other hand, for any $\varepsilon>0$ and $u \in(0,1]$, we can define $g_{u, \varepsilon} \in G_{1}$ by

$$
g_{u, \varepsilon}(x, t)=(1+\varepsilon) x\left[\frac{q(u)}{u} t I_{[0, u]}(t)+q(u) I_{(u, 1]}(t)\right],
$$

for any $(x, t) \in[0,1]^{2}$. Thus, we obtain

$$
\inf _{f \in G_{l}} I(f) \leq \inf _{\varepsilon>0, u \in(0,1]} I\left(g_{u, \varepsilon}\right)=\inf _{0<u \leq 1} \frac{q^{2}(u)}{2 u} .
$$

Therefore (6.2.6) is true. 


\subsection{Large deviations for Kiefer processes}

Let $K:=\{K(x, t), 0 \leq x . t \leq 1\}$ be a Kiefer process, that is a two-parameter Gaussian process with zero mean and covariance function

$$
E K(x . t) K(y . s)=(x \wedge y-x y)(t \wedge s)
$$

It is well known to realize a Kiefer process by letting

$$
K(x, t)=W(x, t)-x W(1, t)
$$

for any $0 \leq x \leq 1$ and $0 \leq t \leq \infty$, where $\{W(x, t), 0 \leq x, t \leq \infty\}$ is a standard Brownian sheet. For $\varepsilon>0$, let $P_{\varepsilon}^{K}$ be a probability measure on $C_{0}[0,1]^{2}$ corresponding to $\sqrt{\varepsilon} K$. Then we have

Theorem 6.3.1 The net of probability measures $\left\{P_{\varepsilon}^{K}, \varepsilon>0\right\}$ satisfies the large deviation principle in q-metric equipped $C_{0}[0,1]^{2}$ with a good rate function $I_{K}: C_{0}[0,1]^{2} \rightarrow$ $[0, \infty]$ that is defined for any $f \in C_{0}[0,1]^{2}$ by

$$
I_{K}(f)= \begin{cases}I(f), & \text { if } I(f)<\infty, \text { and } \sup _{0 \leq t \leq 1}|f(1, t)|=0, \\ \infty, & \text { otherwise. }\end{cases}
$$

Towards proving Theorem 6.3.1, we quote the following contraction principle.

Lemma 6.3.1 (2.1.4 Lemma, Deuschel and Stroock, 1989) Let $X$ and $Y$ be any two metric spaces and $f: X \longrightarrow Y$ a continuous function. Assume that the family of probability measures $\left\{P_{\varepsilon}\right\}$ on $X$ satisfies a large deviation principle with good rate function $I$, then the family of probability measures $\left\{P_{\varepsilon} \circ f^{-1}\right\}$ on $Y$ also satisfies a large deviation principle with good rate function $I^{\prime}$ defined by

$$
I^{\prime}(y)=\inf \{I(x) ; x \in X, y=f(x)\}
$$


Proof of Theorem 6.3.1 Theorem 6.3.1 is a straight consequence of Theorem 6.2.1 and Lemma 6.3.1.

From the latter theorem, we get

Corollary 6.3.1

$$
\lim _{u \rightarrow \infty} \frac{\log P\left(\|K\|_{q} \geq u\right)}{u^{2}}=-\inf _{0<t \leq 1} \frac{2 q^{2}(t)}{t} .
$$

Proof of Corollary 6.3.1 Let $f \in\left\{g: I_{K^{-}}(g)<\infty\right.$ and $\left.\|g\|_{q} \geq 1\right\}$. Then for any $(x, t) \in(0,1) \times(0,1]$

$$
\frac{f^{2}(x, t)}{q^{2}(t)} \leq \frac{1}{2} \int_{0}^{x} \int_{0}^{t}\left(\frac{\partial^{2} f\left(u \cdot v^{\prime}\right)}{\partial u \partial v}\right)^{2} d u d v \cdot \frac{2 x t}{q^{2}(t)}
$$

and

$$
\frac{f^{2}(x, t)}{q^{2}(t)} \leq \frac{1}{2} \int_{x}^{1} \int_{0}^{t}\left(\frac{\partial^{2} f(u, v)}{\partial u \partial v}\right)^{2} d u d v \cdot \frac{2(1-x) t}{q^{2}}
$$

Thus

$$
I_{K}(f) \geq \frac{f^{2}(x, t)}{q^{2}(t)} \inf _{0<t \leq 1} \frac{2 q^{2}(t)}{t}
$$

for any $(x, t) \in[0,1] \times(0,1]$, i.e., we have

$$
I_{K}(f) \geq \inf _{0<t \leq 1} \frac{2 q^{2}(t)}{t} .
$$

On the other hand, for $\varepsilon>0, u \in(0,1]$ let

$$
\begin{aligned}
f_{u, \varepsilon}(x, t) & =2(1+\varepsilon) q(u)\left[\frac{x t}{u} I_{\left[0, \frac{1}{2}\right] \times[0, u]}(x, t)+\frac{(1-x) t}{u} I_{\left(\frac{1}{2}, 1\right] \times[0, u]}(x, t)\right. \\
& \left.+x I_{\left\{0, \frac{1}{2}\right] \times(u, 1]}(x, t)+(x-1) I_{\left(\frac{1}{2}, 1\right] \times(u, 1]}(x, t)\right],
\end{aligned}
$$

for any $(x, t) \in[0,1]^{2}$. Then, we have

$$
\inf _{\|f\|_{q}>1} I_{K}(f) \leq \inf _{\varepsilon>0, u \in(0,1]} I_{K}\left(f_{u, \varepsilon}\right)=\inf _{0<u \leq 1} \frac{2 q^{2}(u)}{u} .
$$

Therefore Corollary 6.3.1 is proved. 
Corollary 6.3.2 Let $F(x)$ be a continuous distribution function on. R. Then

$$
\lim _{u \rightarrow \infty} \frac{\log P\left(\sup _{x \in R} \sup _{0<t \leq 1}\left|K_{F}(x, t)\right| / q(t) \geq u\right)}{u^{2}}=-\inf _{0<t \leq 1} \frac{2 q^{2}(t)}{t} .
$$

Clearly. Corollary 6.3.2 follows from Corollary 6.3.1 immediately.

\subsection{Large deviations for "tied down" Kiefer pro- cesses}

Let $\Gamma:=\{\Gamma(x, t): 0 \leq x, t \leq 1\}$ be a "tied down" Kiefer process, i.e., it is a Gaussian process with zero mean and covariance function

$$
E \Gamma(x, t) \Gamma(y, s)=(x \wedge y-x y)(t \wedge s-t s)
$$

We can realize a "tied down" Kiefer process by letting

$$
\Gamma(x, t)=K(x, t)-t K(x .1)
$$

for any $(x, t) \in[0,1]^{2}$, where $\{K(x, t), 0 \leq x, t \leq 1\}$ is a Kiefer process. For each $\varepsilon>0$, let $P_{\varepsilon}^{\Gamma}$ be a probability measure on $C_{0}[0,1]^{2}$ corresponding to $\sqrt{\varepsilon} \Gamma$. Then we have

Theorem 6.4.1 The net of probability measures $\left\{P_{\varepsilon}^{\Gamma}: \varepsilon>0\right\}$ satisfies the large deviation principle in q-metric equipped $C_{0}[0,1]^{2}$ with the good rate function $I_{\Gamma}$ : $C_{0}[0,1]^{2} \rightarrow[0, \infty]$ that for $f \in C_{0}[0,1]^{2}$ is defined by

$$
I_{\Gamma}(f)= \begin{cases}I(f), & \text { if } I(f)<\infty, \sup _{0 \leq x \leq 1}|f(x, 1)|=0 \text { and } \sup _{0<t \leq 1}|f(1, t)|=0, \\ \infty, & \text { otherwise. }\end{cases}
$$

Corollary 6.4.1

$$
\lim _{u \rightarrow \infty} \frac{\log P\left(\|\Gamma\|_{q} \geq u\right)}{u^{2}}=-\inf _{0<t<1} \frac{2 q^{2}(t)}{t(1-t)}
$$


By the latter corollary and the fact that $\{\Gamma(x, 1-t): 0 \leq x . t \leq 1\}$ is also a "tied down" Kiefer process, we have

Corollary 6.4.2

$$
\lim _{u \rightarrow \infty} \frac{1}{u^{2}} \log P\left(\sup _{0 \leq x \leq 1} \sup _{0 \leq t<1} \frac{|\Gamma(x, t)|}{q(1-t)} \geq u\right)=-\inf _{0<t<1} \frac{2 q^{2}(t)}{t(1-t)}
$$

Corollary 6.4.3 Let $\hat{q}(t)$ be a function defined on $[0,1]$, which satisfies the following conditions:

(i) it is increasing on $\left[0, \frac{1}{2}\right]$, and symmetric with respect to $\frac{1}{2}$.

(ii) for some $c>0$,

$$
\int_{0}^{1} \frac{1}{t(1-t)} \exp \left\{-c \frac{\hat{q}^{2}(t)}{t(1-t)}\right\} d t<\infty .
$$

Then, we have

$$
\lim _{u \rightarrow \infty} \frac{1}{u^{2}} \log P\left(\sup _{0 \leq x \leq 1} \sup _{0<t<1} \frac{|\Gamma(x, t)|}{\hat{q}(t)} \geq u\right)=-\inf _{0<t<1} \frac{2 \hat{q}^{2}(t)}{t(1-t)}
$$

Clearly, we also have

Corollary 6.4.4 Let $F$ be a continuous distribution function on $R$, and $\hat{q}$ satisfies the same conditions as Corollary 6.4.3. Then

$$
\lim _{u \rightarrow \infty} \frac{1}{u^{2}} \log P\left(\sup _{x \in R} \sup _{0<t<1} \frac{\left|\Gamma_{F}(x, t)\right|}{\hat{q}(t)} \geq u\right)=-\inf _{0<t<1} \frac{2 \hat{q}^{2}(t)}{t(1-t)}
$$

Remark 6.4.1 Let $\{B(t), 0 \leq t \leq 1\}$ be a Brownian bridge. Then, under the same assumptions as those of Corollary 6.4 .3 for $\hat{q}$, we also have a similar result as follows

$$
\lim _{u \rightarrow \infty} \frac{1}{u^{2}} \log P\left(\sup _{0<t \leq 1} \frac{|B(t)|}{q(t)} \geq u\right)=-\inf _{0<t<1} \frac{\hat{q}^{2}(t)}{2 t(1-t)}
$$


for any $(x, t) \in[0,1]^{2}$. Then

$$
h_{v, \varepsilon}(x, t) \in\left\{f \in C_{0}[0.1]^{2}: I_{\Gamma}(f)<\infty,\|f\|_{q}>1\right\} .
$$

Hence, we can obtain

$$
\inf _{\|f\|_{q}>1} I_{\Gamma}(f) \leq \inf _{v \in(0.1), \varepsilon>0} I_{\Gamma}\left(h_{v, \varepsilon}\right)=\inf _{0<v<1} \frac{2 q^{2}(v)}{v(1-v)} .
$$

Therefore, the proof is complete.

\section{Proof of Corollary 6.4.3 Let}

$$
q_{1}(t)= \begin{cases}\tilde{q}(t), & 0 \leq t \leq \frac{1}{2} \\ \hat{q}\left(\frac{1}{2}\right), & \frac{1}{2} \leq t \leq 1\end{cases}
$$

and

$$
q_{2}(t)= \begin{cases}\dot{q}\left(\frac{1}{2}\right), & 0 \leq t \leq \frac{1}{2} \\ \hat{q}(t), & \frac{1}{2} \leq t \leq 1\end{cases}
$$

Then, $q_{1}, q_{2}$ satisfy the same conditions as our usual $q$. Hence, by Corollary 6.4.1, 6.4.2, we obtain

$$
\begin{aligned}
& \lim \sup _{u \rightarrow \infty} \frac{1}{u^{2}} \log P\left(\sup _{0 \leq x \leq 1} \sup _{0<t<1} \frac{|\Gamma(x, t)|}{\tilde{q}(t)} \geq u\right) \\
& \leq-\left(\inf _{0<t \leq 1} \frac{2 q_{1}^{2}(t)}{t(1-t)}\right) \wedge\left(\inf _{0 \leq t<1} \frac{2 q_{2}^{2}(t)}{t(1-t)}\right) \\
& =-\inf _{0<t<1} \frac{2 \hat{q}^{2}(t)}{t(1-t)}
\end{aligned}
$$

and

$$
\begin{aligned}
& \liminf \inf _{u \rightarrow \infty} \frac{1}{u^{2}} \log P\left(\sup _{0 \leq x \leq 1} \sup _{0<t<1} \frac{|\Gamma(x, t)|}{\tilde{q}(t)} \geq u\right) \\
& \geq \lim _{u \rightarrow \infty} \frac{1}{u^{2}} \log P\left(\sup _{0 \leq x \leq 1} \sup _{0<t \leq 1} \frac{|\Gamma(x, t)|}{q_{1}(t)} \geq u\right) \\
& =-\inf _{0<t<1} \frac{2 \hat{q}^{2}(t)}{\frac{t(1-t)}{(1-}} .
\end{aligned}
$$

This also completes the proof. 


\section{Chapter 7}

\section{Brownian Local Time Processes}

\subsection{Introduction}

Let $W:=\{W(t), 0 \leq t<\infty\}$ be a standard Brownian motion. For any Borel set $A$ of the real line the random measure

$$
H(A, t)=\lambda\{s: 0 \leq s \leq t . W(s) \in A\},
$$

where $\lambda$ is the Lebesgue measure, is called the occupation time of $W$. It is well known that $H(A, t)$ is absolutely continuous with respect to $\lambda$. The Randon-Nikodym derivative of $H$ is called the local time of $W$ and it will be denoted by $L$, i.e., $\{L(x, t),-\infty<x<\infty, 0 \leq t<\infty\}$ is defined by

$$
H(A, t)=\int_{A} L(x, t) d x .
$$

Trotter (1958) proved that $L(x, t)$ is continuous in both arguments. Concerning the modulus of continuity he proved

$$
\underset{h \rightarrow 0}{\limsup } \sup _{-\infty<x<\infty} \frac{L(x+h, t)-L(x, t)}{h^{1 / 2} \log h^{-1}}=0 \quad \text { a.s. }
$$

for any $t>0$, and

$$
\limsup _{h \rightarrow 0} \sup _{0 \leq t \leq 1-h-\infty<x<\infty} \frac{L(x, t+h)-L(x, t)}{h^{1 / 3}\left(\log h^{-1}\right)^{2 / 3}}=0 \quad \text { a.s. }
$$


The result of (7.1.1) was made precise by H. P. MIcKean .Jr. (1962) and D. B. Ray (1963) as follows:

$$
\limsup _{h \rightarrow 0} \sup _{-\infty<x<\infty} \frac{L(x+h ; t)-L(x, t)}{\left(h \log h^{-1}\right)^{1 / 2}}=2\left(\max _{-\infty<x<\infty} L(x . t)\right)^{1 / 2} \quad \text { a.s. }
$$

They also proved

$$
\limsup _{h \rightarrow 0} \frac{L(h, t)-L(0, t)}{\left(h \log \log h^{-1}\right)^{1 / 2}}=2(L(0 . t))^{1 / 2} \quad \text { a.s. }
$$

for any fixed $t>0$.

For the modulus of continuity in $t$, Hawkes (1971) proved the foilowing result

$$
\lim _{h \rightarrow 0} \frac{\sup _{0 \leq t \leq 1-h}(L(0, h+t)-L(0, t))}{\left(h \log h^{-1}\right)^{1 / 2}}=1 \quad \text { a.s. }
$$

and Perkins (1981) proved that

$$
\limsup _{h \rightarrow 0} \frac{\sup _{0 \leq t \leq 1-h} \sup _{-\infty<x<\infty}(L(x, h+t)-L(x, t))}{\left(2 h \log h^{-1}\right)^{1 / 2}}=1 \quad \text { a.s. }
$$

which, in turn, make (7.1.1) and (7.1.2) precise. Moreover. Kesten (1965) proved the following law of the iterated logarithm:

$$
\limsup _{t \rightarrow \infty} \frac{L(0, t)}{(2 t \log \log t)^{1 / 2}}=\limsup _{t \rightarrow \infty} \frac{\sup _{-\infty<x<\infty} L(x, t)}{(2 t \log \log t)^{1 / 2}}=1 \quad \text { a.s. }
$$

The aim of this chapter is to advance the study of the local time of a Wiener process. In Section 7.2 we discuss increments of the Brownian local time process. Some large deviations results are proved in Section 7.3. Finally, we present and prove Strassen-type laws both for large time and small time.

\subsection{Increments of the Brownian Local Time Pro- cess}

Let $L(t)=: L(0, t)$. Investigating large increments of $L(t)$, Csáki, Csörgö, Földes and Révész (1983) proved the following theorems. 
and

$$
\limsup _{T \rightarrow \infty} \sup _{0 \leq t \leq T-a_{T}-\infty<x<\infty} \sup _{-\infty} \frac{L\left(x, t+a_{T}\right)-L(x, t)}{\left(2 a_{T}\left(\log \frac{T}{a_{T}}+\log \log T\right)\right)^{\frac{1}{2}}}=1 \quad \text { a.s. }
$$

If we also assume that.

$$
\lim _{T \rightarrow \infty} \frac{\log T a_{T}^{-1}}{\log \log T}=\infty
$$

then we also have

$$
\lim _{T \rightarrow \infty} \sup _{0 \leq t \leq T-a_{T}} \sup _{-\infty<x<\infty} \frac{L\left(x, t+a_{T}\right)-L(x, t)}{\left(2 a_{T}\left(\log \frac{T}{a_{T}}+\log \log T\right)\right)^{\frac{1}{2}}}=1 \quad \text { a.s. }
$$

Remark 7.2.2 In case $a_{T}=T$. Theorem 7.2.1 and 7.2.2 imply (7.1.7), the law of the iterated logarithm for the local time of a Wiener process.

Motivated by Hanson and Russo (1983) and Chen, Kong and Lin (1986) (cf. also Step 1 on page 735 in the proof of Theorem 1 in Csörgö and Révész, 1979), we present the following results.

Theorem 7.2.3 Let $0<a_{T} \leq T$ be a function of $T$. Then we have

$$
\limsup _{T \rightarrow \infty} \sup _{a_{T} \leq t \leq T} d(T, t)(L(T)-L(T-t))=1 \quad \text { a.s., }
$$

and

$$
\limsup _{T \rightarrow \infty} \sup _{a_{T} \leq t \leq T} \sup _{t \leq s \leq T} d(T, t)(L(s)-L(s-t))=1 \quad \text { a.s. }
$$

where

$$
\begin{gathered}
d(T, t)=(t(\log (T / t)+2 \log \log t))^{-1 / 2}, \\
\log \log =\log \log (e \vee x) .
\end{gathered}
$$

If we also assume that $a_{T}$ and $T^{-1} a_{T}$ are non-decreasing, and

$$
\lim _{T \rightarrow \infty} \frac{\log T a_{T}^{-1}}{\log \log T}=\infty
$$


then we also have

$$
\lim _{T \rightarrow \infty} \sup _{a_{T} \leq t \leq T} \sup _{t \leq s \leq T} d(T . t)(L(s)-L(s-t))=1 \quad \text { a.s. }
$$

Remark 7.2.3 Replacing $L(0, t)$ by $L(x, t)$, Theorem 7.2.3 remains true for any fixed real number $x$. Moreover, we have a similar result for the largest possible increment in $t$ when $x$ is also varying (cf. Theorem 7.2.4). It is interesting to compare the normalizing factors $d(T, t)$ and $g(T, t)$.

Theorem 7.2.4 Let $0<a_{T} \leq T$ be a function of $T$. Then we have

$$
\limsup _{T \rightarrow \infty} \sup _{a_{T} \leq t \leq T} \sup _{-\infty<x<\infty} g(T, t)(L(x, T)-L(x, T-t))=1 \quad \text { a.s. }
$$

and

$$
\limsup _{T \rightarrow \infty} \sup _{a_{T} \leq t \leq T} \sup _{t \leq s \leq T} \sup _{-\infty<x<\infty} g(T, t)(L(x, s)-L(x, s-t))=1 \quad \text { a.s. }
$$

where

$$
g(T, t)=(2 t(\log (T / t)+\log \log t))^{-1 / 2} .
$$

If we also assume that $a_{T}$ and $T^{-1} a_{T}$ are non-decreasing, and

$$
\lim _{T \rightarrow \infty} \frac{\log T a_{T}^{-1}}{\log \log T}=\infty
$$

then we also have

$$
\lim _{T \rightarrow \infty} \sup _{a_{T} \leq t \leq T} \sup _{t \leq s \leq T} \sup _{-\infty<x<\infty} g(T, t)(L(x, s)-L(x, s-t))=1 \quad \text { a.s. }
$$

Remark 7.2.4 In case $a_{T}=T$, Theorem 7.2.3 and 7.2.4 also imply (7.1.7), the law of the iterated logarithm for the local time of a Wiener process.

To prove our theorems, the following lemmas will be used. 
Lemma 7.2.1 (P. Lévy, 1948).

$$
\left\{\sup _{0 \leq s \leq t} W(s), 0 \leq t<\infty\right\} \stackrel{\mathcal{D}}{=}\{L(t) .0 \leq t<\infty\}
$$

Lemma 7.2.2 (H. Kesten, 1965). For any $\mu>0$, as $x \rightarrow \infty$, we have

$$
P\left(L^{*}(1) \geq x\right)=o\left(\exp \left\{\frac{-x^{2}}{2}(1-\mu)\right\}\right) .
$$

Lemma 7.2.3 (Csáki, Csörgö, Földes and Révész 1983) For any $\varepsilon>0$, there exists $C=C(\varepsilon)>0$ such that

$$
\begin{gathered}
P\left(\sup _{0 \leq t \leq T-V}\left(L\left(t+C_{V}+V\right)-L\left(t+C_{V}\right)>u \sqrt{V}\right)\right. \\
\leq C \sqrt{T / V} \exp \left\{-u^{2} /(2+\varepsilon)\right\}
\end{gathered}
$$

holds true for any $u>0,0 \leq V \leq T$ and $C_{V} \geq 0$.

Lemma 7.2.4 (Csáki, Csörgő, Földes and Révész 1983) For any $\varepsilon>0$, there exists $C=C(\varepsilon)>0$ such that

$$
\begin{gathered}
P\left(\sup _{0 \leq t \leq T-V} \sup _{-\infty<x<\infty}\left(L\left(x, t+C_{V}+V\right)-L\left(x, t+C_{V}\right)>u \sqrt{V}\right)\right. \\
\leq C(T / V) \exp \left\{-u^{2} /(2+\varepsilon)\right\}
\end{gathered}
$$

holds true for any $u>0,0 \leq V \leq T$ and $C_{V} \geq 0$.

Proof of Theorem 7.2.3 Firstly, we show that for any $\varepsilon>0$ we have

$$
\limsup _{T \rightarrow \infty} \sup _{0 \leq t \leq T} \sup _{t \leq s \leq T} d(T, t)(L(s)-L(s-t)) \leq 1+\varepsilon \quad \text { a.s. }
$$

Take $\theta>1$ such that $\eta=\frac{(1+\varepsilon)^{2}}{2+\varepsilon} \frac{1}{\theta}-\frac{1}{2}>0$. Let

$$
T_{n}=2^{n}, \quad K=\left[\log _{\theta} e\right], \quad K_{n}=\left[\log _{\theta} 2^{n+1}\right]+1, \quad K_{n}^{\prime}=\left[\log _{\theta} \frac{2^{n}}{n^{2 / \eta}}\right]+1, \quad t_{k}=\theta^{k}
$$


which, combined with (7.2.16), imply that

$$
\sum_{n=1}^{\infty} P\left(\sup _{-\infty<k \leq K_{n}} A_{n k} \geq 1+\varepsilon\right) \leq \sum_{n=1}^{\infty} \sum_{-\infty<k \leq K_{n}} P\left(A_{n k} \geq 1+\varepsilon\right)<\infty .
$$

Hence, by Borel-Cantelli lemma, we obtain (7.2.15). Letting $\varepsilon \downarrow 0$, we get that

$$
\limsup _{T \rightarrow \infty} \sup _{0 \leq t \leq T} \sup _{t \leq s \leq T} d(T . t)(L(s)-L(s-t)) \leq 1 \quad \text { a.s. }
$$

from (7.2.15). Using (7.1.7), we conclude

$$
\limsup _{T \rightarrow \infty} \sup _{a_{T} \leq t \leq T} d(T, t)(L(T)-L(T-t)) \geq \limsup _{T \rightarrow \infty} d(T, T) L(T)=1 \quad \text { a.s. }
$$

Combining (7.2.18) with (7.2.19), we obtain (7.2.7) and (7.2.8).

$$
\begin{aligned}
& \text { Using Theorem } 7.2 .1 \text {, we have } \\
& \liminf \inf _{T \rightarrow \infty} \sup _{a_{T} \leq t \leq T} \sup _{t \leq s \leq T} d(T, t)(L(s)-L(s-t)) \\
& \geq \lim \inf _{T \rightarrow \infty} \sup _{a_{T} \leq s \leq T} d\left(T, a_{T}\right)\left(L(s)-L\left(s-a_{T}\right)\right) \\
& \geq \lim \inf _{T \rightarrow \infty} \sup _{0 \leq s \leq T-a_{T}}\left[a_{T}\left(\log \left(T / a_{T}\right)+2 \log \log T\right)\right]^{1 / 2}\left(L\left(s+a_{T}\right)-L(s)\right) \\
& =1 \quad \text { a.s. }
\end{aligned}
$$

Hence (7.2.9) is also true. Therefore, the proof of Theorem 7.2 .3 is complete.

Proof of Theorem 7.2.4 Replacing Lemmas 7.2.1 and 7.2.3 by Lemmas 7.2.2 and 7.2.4, respectively, Theorem 7.2.4 can be proved by following the lines of the Proof of Theorem 7.2.3.

\subsection{A Large Deviation}

Let $P_{\varepsilon}^{*}$ be the probability measure on $C_{0}[0,1]$ corresponding to $\left\{\sqrt{\varepsilon} L^{*}(t), 0 \leq t \leq\right.$ $1\}:=\left\{\sqrt{\varepsilon} \sup _{x \in R} L(x, t), 0 \leq t \leq 1\right\}$, and $P_{\varepsilon}(x)$ be the probability measure on 
$C_{0}[0.1]$ corresponding to $\{\sqrt{\varepsilon} L(x, t), 0 \leq t \leq 1\}$ for any fixed $x \in R$. For $f \in C_{0}[0,1]$, we define

$$
I_{0}(f)= \begin{cases}1 / 2 \int_{0}^{1}\left(f^{\prime}(t)\right)^{2} d t, & \text { if } f \text { is an increasing absolutely continuous function } \\ & \text { with a square integrable derivative } f^{\prime} \\ \infty, & \text { otherwise }\end{cases}
$$

and

$$
d(f)= \begin{cases}\inf \{s: 0<s \leq 1, f(s)>0\}, & \text { if }\{s: 0<s \leq 1, f(s)>0\} \neq \emptyset, \\ \infty, & \text { otherwise. }\end{cases}
$$

For any fixed $x \neq 0$, define $I_{x}: C_{0}[0,1] \rightarrow[0, \infty]$ for any $f \in C_{0}[0,1]$ by

$$
I_{x}(f)=\frac{x^{2}}{2 d(f)}+I_{0}(f) .
$$

Our large deviation results for $P_{\varepsilon}(x)$ and $P_{\varepsilon}^{*}$ are as follows:

Theorem 7.3.1 For any fixed $x \in R$, we have that for any closed $\operatorname{set} C \subset C_{0}[0,1]$,

$$
\limsup _{\varepsilon \rightarrow 0} \varepsilon \log P_{\varepsilon}(x)(C) \leq-\inf _{f \in C} I_{x}(f) .
$$

and for any open set $G \subset C_{0}[0,1]$,

$$
\liminf _{\varepsilon \rightarrow 0} \varepsilon \log P_{\varepsilon}(x)(G) \geq-\inf _{f \in G} I_{x}(f)
$$

In addition, for any $x \in R$ and $a \geq 0$, the set $\left\{f: I_{x} \leq a\right\}$ is compact in $C_{0}[0,1]$.

Theorem 7.3.2 For any closed set $C \subset C_{0}[0,1]$, we have

$$
\limsup _{\varepsilon \rightarrow 0} \varepsilon \log P_{\varepsilon}^{*}(C) \leq-\inf _{f \in C} I_{0}(f)
$$


where

$$
\tau_{x}(f)= \begin{cases}\inf \{s: f(s)=x .0 \leq s \leq 1\}, & \text { if }\{s: f(s)=x, 0 \leq s \leq 1\} \neq \emptyset . \\ 1, & \text { otherwise. }\end{cases}
$$

Clearly, $\Pi$ is continuous. By Lemma 6.3.1 and Theorem 2.2.1, Theorem 7.3.1 holds for the rate function

$$
J_{x}(f)= \begin{cases}\inf _{g \in \Pi^{-1}(f)} I(g), & \text { if } \Pi^{-1}(g) \neq \emptyset \\ \infty, & \text { otherwise. }\end{cases}
$$

Therefore, it suffices to show that for any $f \in C_{0}[0,1]$

$$
J_{x}(f)=I_{x}(f)
$$

Since the latter equation is trivial for $f \leq 0$, we assume $f>0$. If $I_{x}(f)<\infty$. let

$$
\vec{f}(t)= \begin{cases}\frac{x}{d(f)} t, & \text { if } 0 \leq t \leq d(f) \\ f(t)+x, & \text { if } d(f) \leq t \leq 1\end{cases}
$$

Then, $\Pi \bar{f}=f$, and

$$
\begin{aligned}
J_{x}(f) & \leq I(\bar{f}) \\
& =\frac{1}{2} \int_{0}^{d(f)}\left(\bar{f}^{\prime}(s)\right)^{2} d s+\frac{1}{2} \int_{d(f)}^{1}\left(\bar{f}^{\prime}(s)\right)^{2} d s \\
& =\frac{x^{2}}{2 d(f)}+I_{0}(f) \\
& =I_{x}(f) .
\end{aligned}
$$

On the other hand, if $J_{x}(f)<\infty$, then for any $g \in \Pi^{-1}(f)$, we have

$$
\begin{aligned}
I(g) & =\frac{1}{2} \int_{0}^{\tau_{x}(g)}\left(g^{\prime}(s)\right)^{2} d s+\frac{1}{2} \int_{\tau_{x}(g)}^{1}\left(g^{\prime}(s)\right)^{2} d s \\
& \geq \frac{x^{2}}{2 \tau_{x}(g)}+\frac{1}{2} \int_{\tau_{x}(g)}^{1}\left(g^{\prime}(s)\right)^{2} I_{\{s: g(s)=\bar{f}(s)\}}(s) d s+\frac{1}{2} \int_{\tau_{x}(g)}^{1}\left(g^{\prime}(s)\right)^{2} I_{\{s: g(s)<\bar{f}(s)\}}(s) d s \\
& \geq \frac{x^{2}}{2 d(f)}+\frac{1}{2} \int_{d(f)}^{1}\left(f^{\prime}(s)\right)^{2} d s \\
& =I_{x}(f) .
\end{aligned}
$$


Hence

$$
J_{x}(f) \geq I_{x}(f)
$$

This completes the proof of Theorem 7.3.1 .

Proof of Theorem 7.3.2 We let $\delta>0$ and define

$$
C^{\delta}=\bigcup_{f \in C} S(f, \delta)
$$

where $S(f, \delta)=\left\{g \in C_{0}[0,1]:\|g-f\|<\delta\right\}$. Define $\pi_{n}: C_{0}[0,1] \rightarrow C_{0}[0,1]$ by $\pi_{n} x(\cdot)$ to be the polygonalization of any function $x(\cdot)$ in $C_{0}[0,1]$ with steps of size $1 / n$. Then

$$
\begin{aligned}
P_{\varepsilon}^{*}(C) & \leq P_{\varepsilon}^{*}\left\{f: \pi_{n} f \in C^{\delta}\right\}+P_{\varepsilon}^{*}\left\{f:\left\|\pi_{n} f-f\right\| \geq \delta\right\} \\
& :=A+B
\end{aligned}
$$

Take $d_{\delta}=\inf _{f \in C^{\delta}} I(f)$. Then

$$
\begin{aligned}
A & =P_{\varepsilon}^{*}\left(\pi_{n} f \in C^{\delta}\right) \\
& \leq P_{\varepsilon}^{*}\left(I\left(\pi_{n} f\right) \geq d_{\delta}\right) \\
& =P_{\varepsilon}^{*}\left(\frac{1}{2} \sum_{i=1}^{n}\left(\frac{f\left(\frac{i}{n}\right)-f\left(\frac{i-1}{n}\right)}{\frac{1}{n}}\right)^{2} \frac{1}{n} \geq d_{\delta}\right) \\
& =P\left(n \sum_{i=1}^{n}\left(\sqrt{\varepsilon} L^{*}\left(\frac{i}{n}\right)-\sqrt{\varepsilon} L^{*}\left(\frac{i-1}{n}\right)\right)^{2} \geq 2 d_{\delta}\right) \\
& \leq P\left(\sum_{i=1}^{n}\left(\sup _{x \in R}\left(L\left(x, \frac{i}{n}\right)-L\left(x, \frac{i-1}{n}\right)\right)\right)^{2} \geq \frac{2 d_{\delta}}{n \varepsilon}\right) \\
& =P\left(\sum_{i=1}^{n}\left(\sup _{x \in R}(L(x, i)-L(x, i-1))\right)^{2} \geq \frac{2 d_{\delta}}{\varepsilon}\right) \\
& :=P\left(\sum_{i=1}^{n} X_{i}^{2} \geq 2 d_{\delta} / \varepsilon\right),
\end{aligned}
$$

where $X_{1}, X_{2}, \ldots, X_{n}$ are i.i.d.r.v.s., and by Lemma 7.2 .2 , for any $\mu>0$, as $x \rightarrow \infty$,

$$
P\left(X_{1} \geq x\right)=o\left(\exp \left\{-\frac{(1-\mu) x^{2}}{2}\right\}\right) .
$$


It is easy to show that $E e^{t X_{1}^{2}}<\infty$ for $t \in\left[0 . \frac{1-2 \mu}{2}\right]$. Thus

$$
\begin{aligned}
A & \leq P\left(\exp \left\{\frac{1-2 \mu}{2} \sum_{i=1}^{n} X_{i}^{2}\right\} \geq \exp \left\{2 \cdot \frac{(1-2 \mu) d_{\delta}}{2} / \varepsilon\right\}\right) \\
& \leq \exp \left\{-(1-2 \mu) d_{\delta} / \varepsilon\right\}\left(E \exp \left\{\frac{1-2 \mu}{2} X_{1}^{2}\right\}\right)^{n} .
\end{aligned}
$$

Therefore,

$$
\begin{aligned}
\limsup _{\varepsilon \rightarrow 0} \varepsilon \log A & \leq \limsup _{\varepsilon \rightarrow 0} \varepsilon\left[-\frac{(1-2 \mu) d_{\delta}}{\varepsilon}+n \log E \exp \left\{\frac{1-2 \mu}{2} X_{1}^{2}\right\}\right] \\
& \leq-(1-2 \mu) d_{\delta} .
\end{aligned}
$$

Letting $\mu \rightarrow 0$, we obtain

$$
\limsup _{\varepsilon \rightarrow 0} \varepsilon \log A \leq-d_{\delta}
$$

On the other hand, by Lemma 7.2 .2 , we have

$$
\begin{aligned}
B & \leq P_{\varepsilon}^{*}\left\{\max _{1 \leq i \leq \pi}\left(f\left(\frac{i}{n}\right)-f\left(\frac{i-1}{n}\right)\right) \geq \delta\right\} \\
& \leq \sum_{i=1}^{n} P\left\{\left(L^{*}\left(\frac{i}{n}\right)-L^{*}\left(\frac{i-1}{n}\right)\right) \geq \delta / \sqrt{\varepsilon}\right\} \\
& \leq n P\left(L^{*}(1) \geq \sqrt{n} \delta / \sqrt{\varepsilon}\right) \\
& =o\left(n \exp \left\{-\frac{1-\mu}{2} \cdot \frac{\delta^{2} n}{\varepsilon}\right\}\right) . \quad(\varepsilon \rightarrow 0) .
\end{aligned}
$$

Therefore,

$$
\limsup _{\varepsilon \rightarrow 0} \varepsilon \log B \leq-\frac{1-\mu}{2} \delta^{2} n
$$

and letting $\mu \rightarrow 0$, we get

$$
\limsup _{\varepsilon \rightarrow 0} \varepsilon \log B \leq-\frac{1}{2} \delta^{2} n
$$


Combining(7.3.7)and (7.3.8) with (7.3.6), we obtain

$$
\begin{aligned}
& \limsup _{\varepsilon \rightarrow 0} \varepsilon \log P_{\varepsilon}^{*}(C)=\limsup _{\varepsilon \rightarrow 0} \varepsilon \log (A+B) \\
& \leq \limsup _{\varepsilon \rightarrow 0} \varepsilon[(\log A) \vee(\log B)] \\
& \leq\left(-d_{\delta}\right) \vee\left(-\frac{1}{2} \delta^{2} n\right) \\
& =-\left(d_{\delta} \wedge \frac{\delta^{2} n}{2}\right) \text {. }
\end{aligned}
$$

Let $\delta>0$ be fixed. Then, as $n \rightarrow \infty$,

$$
\limsup _{\varepsilon \rightarrow 0} \varepsilon \log P_{\varepsilon}^{*}(C) \leq-d_{\delta}
$$

For any closed set $C$, it is easy to show that

$$
\lim _{\delta \downarrow 0} d_{\delta}=\inf _{f \in C} I(f)
$$

Thus, if we let $\delta \rightarrow 0$, via (7.3.9) we obtain (7.3.3). The proof of Theorem 7.3.2 is now complete.

\subsection{Functional Limit Theorems}

Let $b_{n}=\sqrt{2 n \log \log n}$, and $\mathcal{S}_{1}:=\left\{f \in C_{0}[0,1]: I_{0}(f) \leq \frac{1}{2}\right\}$. Via the classic Strassen's law (Strassen 1964), Csáki and Révész(1983) obtained the following result.

Theorem 7.4.1 With probability one, the sequence $\left\{b_{n}^{-1} L(n t), 0 \leq t \leq 1\right\}_{n=3}^{\infty}$ is relatively compact in $C_{0}[0,1]$, and the set of its limit points is $\mathcal{S}_{1}$.

We are now interested to extend the latter result. Naturally, we have

Theorem 7.4.2. For any fixed $x \in R$, the sequence $\left\{b_{n}^{-1} L(x, n t), 0 \leq t \leq 1\right\}_{n=3}^{\infty}$ is relatively compact in $C_{0}[0,1]$, with probability one, and the set of its limit points is $\mathcal{S}_{1}$. 
Lemma 7.4.2. For any $x \in R$,

$$
\lim _{n \rightarrow \infty} b_{n}^{-1} \sup _{0 \leq t \leq n}|L(t)-L(x, t)| \stackrel{\text { a.s. }}{=} 0 .
$$

Proof of Lemma 7.4.2 It suffices to show that for $x>0$,

$$
\lim _{n \rightarrow \infty} b_{n}^{-1} \sup _{0 \leq r \leq L(0, n)}\left|r-L\left(x, T_{r}\right)\right| \stackrel{\text { a.s. }}{=} 0 .
$$

First of all, by Lemma 7.4 .1 and a maximal inequality for submartingales, for any $\varepsilon>0$ we have

$$
\begin{aligned}
P\left(\sup _{0 \leq r \leq 2 b_{n}}\left(r-L\left(x, T_{r}\right)\right) \geq \varepsilon b_{n}\right) & \leq \exp \left\{(2-\varepsilon) b_{n} u-2 b_{n}(1+2 x u)^{-1} u\right\} \\
& =\exp \left\{\frac{1}{2 x}(2 \sqrt{4-2 \varepsilon}+\varepsilon-4) b_{n}\right\},
\end{aligned}
$$

where $u=\frac{1}{2 x}\left(\sqrt{\frac{2}{2-\varepsilon}}-1\right)$. Hence, via the Borel-Cantelli lemma, for any $\varepsilon>0$. we obtain

$$
\limsup _{n \rightarrow \infty} b_{n}^{-1} \sup _{0 \leq r \leq 2 b_{n}}\left(r-L\left(x, T_{r}\right)\right) \leq \varepsilon, \quad \text { a.s. }
$$

Similarly, we have

$$
\limsup _{n \rightarrow \infty} b_{n}^{-1} \sup _{0 \leq r \leq 2 b_{n}}\left(L\left(x, T_{r}\right)-r\right) \leq \varepsilon, \quad \text { a.s. }
$$

Combining (7.4.3) with (7.4.4), we arrive at

$$
\lim _{n \rightarrow \infty} b_{n}^{-1} \sup _{0 \leq r \leq 2 b_{n}}\left|r-L\left(x, T_{r}\right)\right| \stackrel{\text { a.s. }}{=} 0 .
$$

Therefore, via the law of the iterated logarithm for $L(0, n)$, we get (7.4.2) from (7.4.5). The proof of Lemma 7.4.2 is now complete.

Proof of Theorem 7.4.2 Theorem 7.4.2 follows from Theorem 7.4.1 and Lemma 7.4.2. 
Towards proving Theorem 7.4 .3 . we let $\theta>1$, and define

$$
y=y_{\theta}(T)=\frac{T \log T}{a_{T}} \theta
$$

Let $T_{\theta}(y)$ be the inverse of the function $y_{\theta}(T)$. We prove the following lemma.

Lemma 7.4.3 Under the assumptions of Theorem 7.4.3, for $a_{T}$, we have

$$
\lim _{\theta \rightarrow \infty} \sup _{c(\theta) \leq y \leq \infty} \frac{T_{\theta}(y+1)}{T_{\theta}(y)}=1
$$

and

$$
\lim _{\theta \rightarrow \infty} \sup _{c(\theta) \leq y \leq \infty} \frac{T_{\theta}(y+1)-T_{\theta}(y)}{a_{T_{\theta}(y)}}=0
$$

where $c(\theta)$ is some constant that depends on $\theta$.

Proof of Lemma 7.4.3 On account of

$$
\frac{d y}{d T}=\frac{(\log T+1) a_{T}-a_{T}^{\prime} T \log T}{a_{T}^{2}} \theta
$$

we have

$$
\frac{d T_{\theta}(y)}{d y}=\frac{a_{T_{\theta}(y)}^{2}}{\left(\log T_{\theta}(y)+1\right) a_{T_{\theta}(y)}-a_{T_{\theta}(y)}^{\prime} T_{\theta}(y) \log T_{\theta}(y)} \frac{1}{\theta} .
$$

On noting

$$
0 \leq a_{T}^{\prime} \leq \frac{a_{T}}{T}
$$

we have

$$
\frac{d T_{\theta}(y)}{d y} \leq \frac{a_{T_{\theta}(y)}}{\theta}
$$


For large enough $T$, there exists $k \in N$ such that.

$$
T_{k} \leq T<T_{k+1}
$$

Thus

$$
\begin{aligned}
\left\|\eta_{T}^{*}(\cdot)-\eta_{T_{k}}^{*}(\cdot)\right\| \leq & \sup _{x \in R} \sup _{0 \leq t \leq 1} \beta_{T_{k}} \mid\left(L\left(x, T-a_{T}+t a_{T}\right)-L\left(x, T-a_{T}\right)\right) \\
& -\left(L\left(x, T_{k}-a_{T_{k}}+t a_{T_{k}}\right)-L\left(x \cdot T_{k}-a_{T_{k}}\right)\right) \mid \\
& +\sup _{x \in R} \sup _{0 \leq t \leq 1}\left|\left(\beta_{T}-\beta_{T_{k}}\right)\left(L\left(x . T-a_{T}+t a_{T}\right)-L\left(x, T-a_{T}\right)\right)\right| \\
\leq & 2 \beta_{T_{k}} \sup _{x \in R} \sup _{0 \leq s \leq T_{k}}\left(L\left(x, s+T_{k+1}-T_{k}\right)-L(x, s)\right) \\
& +\left(\frac{\beta_{T_{k}}}{\beta_{T_{k+1}}}-1\right) \beta_{T} \sup _{x \in R}\left(L(x, T)-L\left(x . T-a_{T}\right)\right) \\
:= & E+F .
\end{aligned}
$$

Via Theorem 7.2.2, we have

$$
\limsup _{k \rightarrow \infty} \sup _{x \in R} \sup _{0 \leq s \leq T_{k}} \frac{L\left(x, s+T_{k+1}-T_{k}\right)-L(x, s)}{\left[2\left(T_{k+1}-T_{k}\right)\left(\log \frac{T_{k+1}}{T_{k+1}-T_{k}}+\log \log T_{k+1}\right)\right]^{\frac{1}{2}}} \leq 1, \quad \text { a.s., }
$$

and, by Lemma 7.4.3,

$$
\lim _{\theta \rightarrow \infty} \beta_{T_{k}}\left[2\left(T_{k+1}-T_{k}\right)\left(\log \frac{T_{k+1}}{T_{k+1}-T_{k}}+\log \log T_{k+1}\right)\right]^{\frac{1}{2}}=0 .
$$

Thus taking $\theta$ and $k$ large enough, respectively, we get

$$
E<\delta, \quad \text { a.s. }
$$

Similarly, taking again $\theta$ and $k$ large enough, respectively, we have also

$$
F<\delta, \quad \text { a.s. }
$$

Therefore, taking $\theta$ and $k$ large enough, we obtain

$$
\left\|\eta_{T}^{*}(\cdot)-\eta_{T_{k}}^{*}(\cdot)\right\|<2 \delta, \quad \text { a.s. }
$$


Combining ( 7.4 .8$)$ with (7.4.9), we get

$$
P\left(\bigcup_{M>1} \bigcap_{T>M}\left\{\eta_{T}^{*}(\cdot) \in \mathcal{S}^{3 \delta}\right\}=1\right.
$$

This also completes the proof of Theorem 7.4.3.

\subsection{The Small Time Strassen Law}

In this section. we consider another application of the large deviations obtained in Section 1. Gantert(1993) proved the small time Strassen law for Brownian motion (cf. Section 2.4).

Let

$$
\begin{array}{ll}
\xi_{h}^{*}(t)=\frac{L^{*}(h t)}{\sqrt{2 h \log \log \frac{1}{h}}}, & 0 \leq t \leq 1, \\
\xi_{h}(t)=\frac{L(h t)}{\sqrt{2 h \log \log \frac{1}{h}}}, & 0 \leq t \leq 1 .
\end{array}
$$

Using Theorems 7.3.2 and Theorem 2.2.1, we can also prove the following local versions of Strassen's law for Brownian local times. The proofs, which are similar to that of Theorem 7.4.1 and 7.4.2, are ommited.

Theorem 7.5.1 With probability one, the set of functions $\left\{\xi_{h}^{*}(\cdot)\right\}$ is relatively compact in $C_{0}[0,1]$, and the set of its limit points is contained in $\mathcal{S}_{1}$.

Theorem 7.5.2 With probability one, the set if functions $\left\{\xi_{h}(0, \cdot), 0<h \leq 1\right\}$ is relatively compact in $C_{0}[0,1]$, and the set of its limit points is $\mathcal{S}_{1}$. 
Clearly, the above results imply the following local versions of the law of the iterated logarithm.

Corollary 7.5.1

$$
\limsup _{h \rightarrow 0} \frac{L(h)}{\sqrt{2 h \log \log \frac{1}{h}}}=1 \quad \text { a.s. }
$$

and

$$
\limsup _{h \rightarrow 0} \frac{L^{*}(h)}{\sqrt{2 h \log \log \frac{1}{h}}}=1 \quad \text { a.s. }
$$


[8] Chen. B. (1990). How small are (H-R) increments of the local time of a Wiener process. J. of Hangzhou University 17. No. 2.

[9] Chen, B. (1991). The set of limit points of increments of the local time of a Wiener process. J. of Hangzhou University 18, No. 3.

[10] Chen, B. (1997). Large deviations and Strassen's limit points of Brownian local time processes. Statistics and Probability Letters 34, 385-395.

[11] Chen, B. (1998). On Strassen's version of the law of the ite ated logarithm for the two-parameter Wiener process. In:Asymptotic Methods in Probability and Statistics - a Volume in Honour of Miklós Csörgö, (B. Szyszkowicz, ed.). Elsevier Science B.V.

[12] Chen, B. and He, F. (1989). Some results on increments of a Wiener process. Chinese J. of Applied Probab. and Statistics 5, No. 4.

[13] Chen, G., Kong, F. and Lin, Z. (1986). Answers to some questions about increments of a Wiener process.Ann. Probab. 14. 1252-1261.

[14] Csáki, E., Csörgö, M., Földes, A. and Révész, P. (1983). How big are the increments of the local time of a Wiener process? Ann. Probab. 11, 593-608.

[15] Csáki, E. and Révész, P. (1983). A combinatorical proof of P. Lévy on the local time, Acta. Sci. Math. Szeged 45, 119-129.

[16] Csörgö, M., Csörgö, S., Horváth, L. and Mason, D. M. (1986). Weighted empirical and quantile processes. Ann. Probab. 14, 31-85.

[17] Csörgö, M., Horváth, L. and Szyszkowicz, B. (1997). Integral tests for supreme of Kiefer processes with application. Statistics and Decisions 15, 365-377. 
[18] Csörgö. M. and Révész, P. (1979). How big are the increments of a Wiener process? Ann. Probab. 7, 731-737.

[19] Csörgö. M. and Révész, P. (1979a). How small are the increments of a Wiener process? Stochastic Processes Appl. 8, 119-129.

[20] Csörgö. M. and Révész, P. (1981). Strong Approximations in Probability and Statistics. Academic Press, New York.

[21] M. Csörgö. Q. Shao and B. Szyszkowicz (1991). A note on local and global functions of a Wiener process and some Rényi-type statistics. Studia Scientiarum Mathematicarum Hungarica 26, 239-259.

[22] Csörgö, M. and Szyszkowicz, B. (1994). Applications of multi-time parameter processes to change-point analysis, In: Probability Theory and Mathematical Statistics - Proceedings of the Sixth Vilnius Conference, pp. 159-222, B. Grigelionis et al. (Eds.), VSP/TEV .

[23] Darkhovsky, D. S. (1984). On two problems of estimating the moments of changes in probabilistic characteristics of a random sequence (in Russian). Teor. Veroyatn. Primen. 29, 464-473.

[24] Deshayes, J. and Picard, D. (1986). Off-line statistical analysis of change-point models using nonparameter and likelihood methods. in: Detection of Abrupt Changes in Signals and Dynamical Systems (M. Thoma and A. Wyner, eds., M. Basseville and A. Beneveniste, guest eds.), pp. 103-168.

[25] Deuschel, J.D. and Stroock, D.W. (1989). Large Deviations. Academic Press, Boston. 
[36] Knight, F. (1981). Essentials of Brownian motion and diffusion. Mathematical Survey Math. Society, Providence. Rhode Island.

[37] Komlós, J., Major, P. and Tusnády, G. (1975). An approximation of partial sums of independent R. V.'s and the sample DF. I. Z. Wahrscheinlichkeitstheorie verw. Gebiete 32, 111-131.

[38] Komlós, J., Major, P. and Tusnády, G. (1976). An approximation of partial sums of independent R. V.'s and the sample DF. II. Z. Wahrscheinlichkeitstheorie verw. Gebiete 34, 33-58.

[39] Lacey, M. (1990). Large deviations for the maximum local time of stable Lévy processes, Ann. Probab. 18, 1669-1675.

[40] Lévy, P. (1937). Théorie de l'addition des variables aléatoires. Gauthier-Villars. Paris.

[41] Lévy, P.(1948). Processus Stochastiques et Mouvement Brownien. GauthierVillars, Paris.

[42] Lin, Z. and Lu, C. (1992). Strong Limit Theorems Science Press, Beijing.

[43] Marcus, M.B. and Rosen, J. (1994). Laws of the iterated logarithm for the local times of symmetric Lévy processes and recurrent random walk, Ann. Probab. 22, 626-658.

[44] McKean, H.P.Jr. (1962). A Hölder condition for Brownian local time. J. Math. Kyoto $U$ 1, 195-201.

[45] Park, W.J. (1974), On Strassen's version of the law of the iterated logarithm for the two-parameter Gaussian process. J. Multivariate Analysis 4, 479-485. 
[5i] Szyszkowicz. B. (1991). Empirical type processes and contiguity. C.R. Math. Rep. Acad. Sci. Canada 13, 161-166.

[58] Szyszkowicz. B. (1994). Weak convergence of weighted empirical type processes under contiguous and changepoint alternatives. Stochastic, Process. Appl. 50 , 281-313.

[59] Trotter. H.F. (1958). A property of Brownian motion paths. Illinois J. Math. 2, 425-433.

[60] Vasudeva,R. and Savitha S. (1993). On the increments of a Wiener process -A look through subsequences. Stochastic Process Appl. 47, 153-158.

[61] Varadhan.S.R.S. (1984). "Large Deviations and Applications" Society for Industrial and Applied Math., Philadelphia.

[62] Wichura, M.J. (1973). Some Strassen-type laws of the iterated logarithm for multiparameter stochastic processes with independent increments. Ann. of Probab. 1, 272-296.

[63] Wiener, N. (1923). Differential space. J. Math. and Phys. 2, 132-174.

[64] Yeh, J. (1963). Cameron-Martin translation theorems in the Wiener space of functions of two variables. Amer. Math. Soc. Trans. 107, 409-420.

[65] Zimmermann, G. (1972). Some sample function properties of the two-parameter Gaussian process. Ann. Math. Statist. 43, 1235-1246. 
